THIAGO FREITAS DA SILVA

CONTRIBUIÇÃO DAS ARMADURAS DE COMPRESSÃO EM CHAPAS DE CONCRETO ARMADO 
THIAGO FREITAS DA SILVA

\title{
CONTRIBUIÇÃO DAS ARMADURAS DE COMPRESSÃO EM CHAPAS DE CONCRETO ARMADO
}

\author{
Dissertação apresentada à Escola \\ Politécnica da Universidade de São Paulo \\ para obtenção do título de Mestre em \\ Engenharia
}


THIAGO FREITAS DA SILVA

\section{CONTRIBUIÇÃO DAS ARMADURAS DE COMPRESSÃO EM CHAPAS DE CONCRETO ARMADO}

Dissertação apresentada à Escola Politécnica da Universidade de São Paulo para obtenção do título de Mestre em Engenharia

Área de Concentração:

Engenharia de Estruturas

Orientador: Prof. Dr. João Carlos Della Bella 
Este exemplar foi revisado e alterado em relação à versão original, sob responsabilidade única do autor e com a anuência de seu orientador.

São Paulo, de julho de 2012.

Assinatura do autor

Assinatura do orientador

FICHA CATALOGRÁFICA

Silva, Thiago Freitas da

Contribuição das armaduras de compressão em chapas de concreto armado / T.F. da Silva. -- ed.rev. -- São Paulo, 2012. $115 \mathrm{p}$.

Dissertação (Mestrado) - Escola Politécnica da Universidade de São Paulo. Departamento de Engenharia de Estruturas e Geotécnica.

1. Concreto armado (Dimensionamento) 2. Chapas 3. Estruturas de membranas I. Universidade de São Paulo. Escola Politécnica. Departamento de Engenharia de Estruturas e Geotécnica II. t. 


\section{AGRADECIMENTOS}

Ao meu orientador, Prof. Dr. João Carlos Della Bella, pelo apoio, paciência e dedicação ao longo do trabalho.

As empresas EGT Engenharia e Cláudio Puga \& Eng. Associados pela compreensão na tarefa de conciliar trabalho e estudos.

A todos os meus amigos pelo carinho e amizade por todos esses anos.

Aos meus pais, Dilma Araújo de Freitas e Miguel Horácio Rodrigues da Silva, pelo amor, dedicação e carinho.

A todos que colaboraram direta ou indiretamente na execução deste trabalho.

A Deus. 
"O valor das coisas não está no tempo que elas duram, mas na intensidade com que acontecem. Por isso, existem momentos inesquecíveis, coisas inexplicáveis e pessoas incomparáveis." (Autor desconhecido) 


\section{RESUMO}

Este trabalho apresenta métodos de dimensionamento analíticos de armaduras de compressão para chapas de concreto com malha de armadura ortogonal. É feita uma revisão bibliográfica mostrando a evolução do estudo das chapas e citando os métodos analíticos de dimensionamento propostos. Estes métodos, em geral, definem como quantificar a armadura necessária para equilibrar os esforços de tração e verificar se a compressão no concreto atende ao limite de resistência. Para os casos em que a compressão na chapa é excessiva, uma das soluções possíveis seria a adoção de armaduras que funcionam comprimidas. Entretanto, não há muita informação na literatura para dimensionamento para estas situações. Assim, é proposto um procedimento para determinação dessas armaduras que se fundamenta no método baseado nos critérios utilizados por Baumann, o qual é apresentado neste trabalho. Neste trabalho são utilizados como limites de resistência à compressão aqueles recomendados pelo CEB (1990), porém, é proposto um modelo em que este limite varia de acordo com a deformação de tração que ocorre perpendicularmente a compressão atuante. Este modelo resistente é baseado nos conceitos propostos por Vecchio e Collins (1986).

Palavras-chave: concreto armado. dimensionamento. chapas. armaduras de compressão. 


\begin{abstract}
This work presents a method to design membrane concrete elements with orthogonal mesh of reinforcement which are subjected to compression stress. A literature review was carried of and it shows the evolution of membrane elements research, pointing the design analytic methods proposed. These methods, in general, define a way to quantify the reinforcement necessary to support the tension stress and verify if the compression in concrete respects the strength limit. In case which the compression in membrane is excessive, it is possible use reinforcements subject to compression. However, there is no much information in literature about how design reinforcement for these cases. Thus, this paper presents a procedure with this objective which it use the model based on Baumann's criteria as basis. In this paper, it are used as limits of strength those recommended by CEB (1990), however, it is proposed a model which this limit vary according to tension deformation which occurs perpendicular to compression. This model of strength is based on concepts proposed by Vecchio e Collins (1986).
\end{abstract}

Keywords: concrete. design. membrane. compression reinforcement. 


\section{LISTA DE ILUSTRAÇÕES}

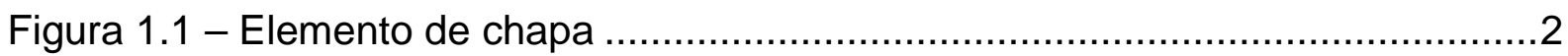

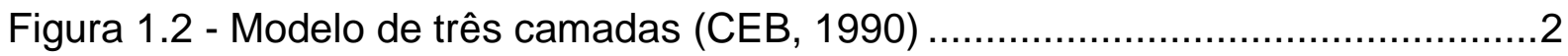

Figura 1.3 - Exemplos de utilização de elementos de chapas em estruturas

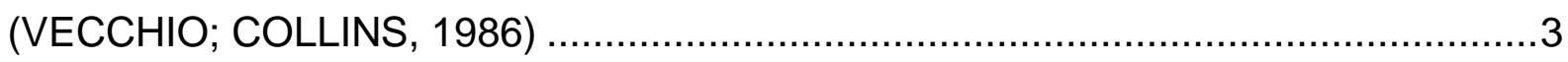

Figura 1.4 - Padrões de posicionamento para armaduras (GUPTA, 1984) ................4

Figura 2.1 - Efeito de pino (adaptado do CEB, 1990).........................................

Figura 2.2 - Engrenamento dos agregados (VECCHIO E COLLINS, 1986)...............8

Figura 2.3 - Acréscimo de rigidez devido ao efeito do "tension-stiffening" (adaptado

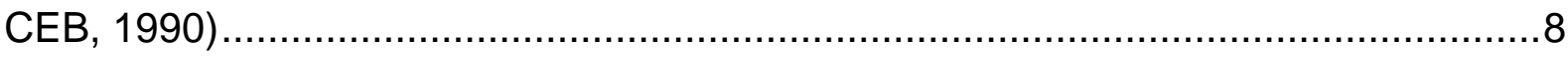

Figura 2.4 - Amolecimento do concreto fissurado (VECCHIO E COLLINS, 1986) ......9 Figura 2.5 - Modelo para resistência biaxial do concreto C30 (KUPFER et al. ,1969

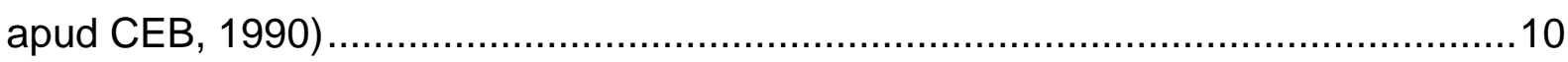

Figura 2.6 - Modelo para elemento de membrana proposto por Nielsen: (a) esforções solicitantes; (b) contribuição da armadura; (c) contribuição do concreto (CHEN, 2004)

Figura 2.7 - Experimento de Vecchio e Collins (1982) ........................................12

Figura 2.8 - Quadro resumo do MCFT (VECCHIO E COLLINS, 1986) ....................14

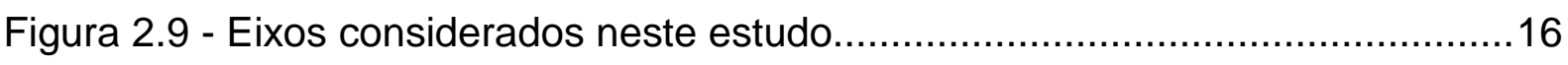

Figura 2.10 - Equilíbrio de forças na seção paralela à fissura.................................17

Figura 2.11 - Equilíbrio de forças na seção perpendicular à fissura.........................18

Figura 2.12 - Deformação da seção paralela a fissura ...........................................20

Figura 2.13 - Configuração deformada e fissuras em chapa submetida a esforço de

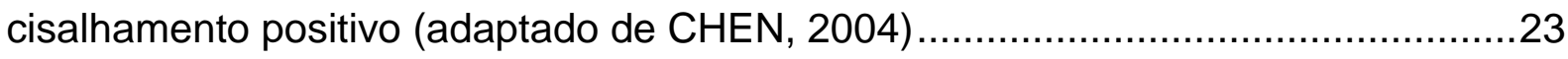

Figura 2.14 - Configuração deformada e fissuras em chapa submetida a esforço de cisalhamento negativo (adaptado de CHEN, 2004) ….......................................23

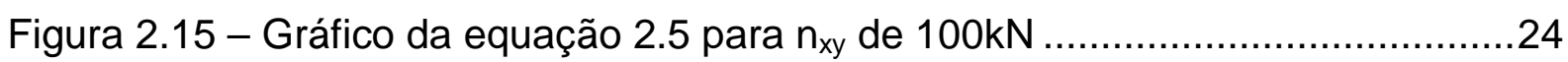

Figura 2.16 - Diagrama tensão-deformação para o aço (NBR-6118:2003) ...............25

Figura 2.17 - Resumo dos casos de dimensionamento de chapas (adaptado CEB, 1990) 
Figura 2.18 - Equilíbrio de forças na seção perpendicular a força de compressão do concreto

Figura 3.1 - Diagrama tensão-deformação parábola-retângulo para o concreto comprimido uniaxialmente (CEB, 1990) .

Figura 3.2 - Representação tridimensional do diagrama tensão-deformação do concreto em compressão (VECCHIO E COLLINS, 1986)

Figura 3.3 - Redução da tensão de pico à compressão do concreto (VECCHIO E COLLINS, 1986)

Figura 3.4 - Diagrama tensão-deformação para o concreto fissurado em compressão

Figura 3.5 - Resistência máxima à compressão do concreto em função da deformação de tração perpendicular .40

Figura 3.6 - Diagrama tensão-deformação 41

Figura 3.7 - Deformação no elemento de chapa (JAZRA, 2008). .42

Figura 3.8 - Sentido positivo das deformações 42

Figura 3.9 - Círculo de Mohr das deformações. 43

Figura 3.10 - Exemplo 3.1 - Tensão de compressão menor que $f_{\text {cd2 }}$ 49

Figura 3.11 - Exemplo 3.2 - Tensão de compressão entre $f_{c d 1}$ e $f_{c d 2}$ e abaixo de $f_{c 2 m a x}$

Figura 3.12 - Exemplo 3.3 - Tensão de compressão entre $f_{c d 1}$ e $f_{c d 2}$ e acima de $f_{c 2 m a x}$ .54

Figura 3.13 - Exemplo 3.4 - Tensão de compressão maior que $\mathrm{f}_{\mathrm{cd} 1}$ .56

Figura 4.1 - Resistência à compressão do concreto em função de $\theta$ para o caso III60

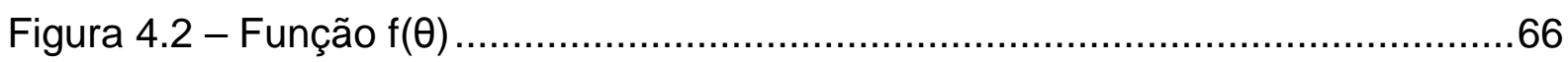

Figura 4.3 - Exemplo 4.1 - Tensão de compressão maior que fcd2 …....................70

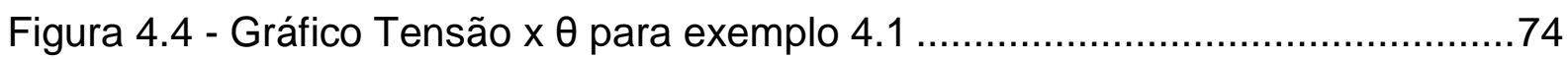

Figura 4.5 - Exemplo 4.2 - Tensão de compressão igual a fcd2 ……....................75

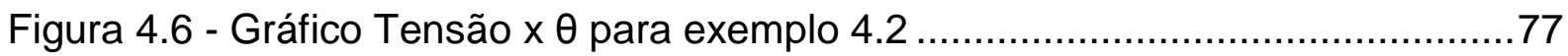

Figura 4.7 - Exemplo 4.3 - Tensão de cisalhamento maior que o limite ....................79

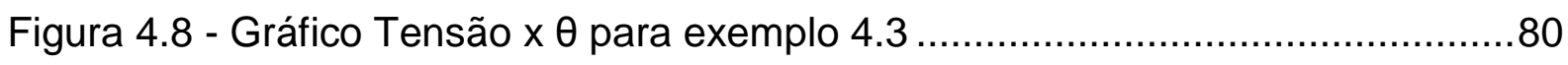

Figura 4.9 - Exemplo 4.4 - Problema com solução inexistente..................................82

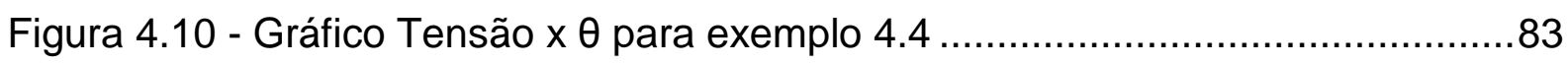

Figura 4.11 - Resistência à compressão do concreto em função de $\theta$ para o caso II 
Figura 5.1 - Equilíbrio da chapa em um trecho de chapa com uma das faces perpendicular a direção principal de maior tensão de compressão.

Figura 5.2 - Equilíbrio da chapa em um trecho de chapa com uma das faces perpendicular a direção principal de menos tensão de compressão.........................90

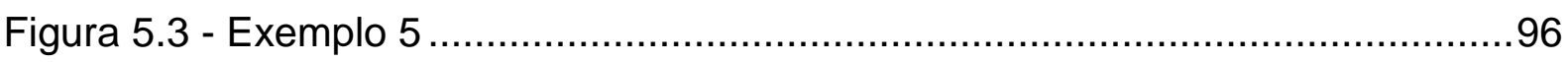

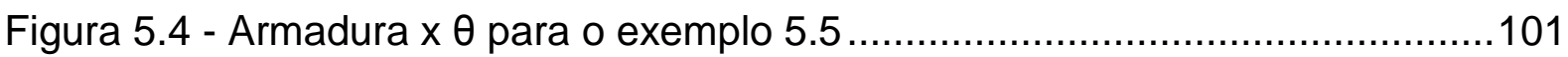

Figura 6.1 - Área de armadura em função do esforço normal $n_{x}$ para o caso III .....103

Figura 6.2 - Área de armadura em função do esforço normal $n_{y}$ para o caso III .....104

Figura 6.3 - Ângulo $\theta$ em função do esforço de cisalhamento para caso III ............104

Figura 6.4 - Esforço nas armaduras em função do esforço de cisalhamento para o

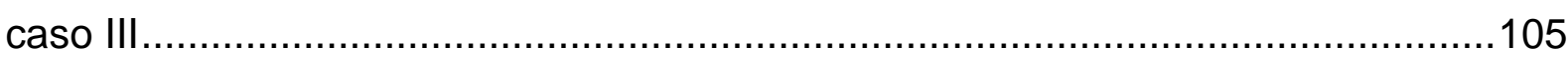

Figura 6.5 - Deformação $\varepsilon_{y}$ em função do esforço de cisalhamente para o caso III 106

Figura 6.6 - Área de armadura em função do esforço de cisalhamento para o caso III 106

Figura 6.7 - Ângulo $\theta$ em função do esforço normal para caso IV..........................107

Figura 6.8 - Área de armadura em função do esforço normal $n_{x}$ para o caso IV .....108 Figura 6.9 - Área de armadura em função do esforço normal $n_{y}$ para o caso IV .....108 Figura 6.10 - Área de armadura em função do esforço de cisalhamento $\mathrm{n}_{\mathrm{xy}}$ para o caso IV 109

Figura 6.11 - Gráfico da área de armadura em função de $\theta$ correspondente a Tabela 6.1 110 


\section{LISTA DE TABELAS}

Tabela 2.1 - Resistências dos aços e deformação de escoamento (NBR 6118).......25

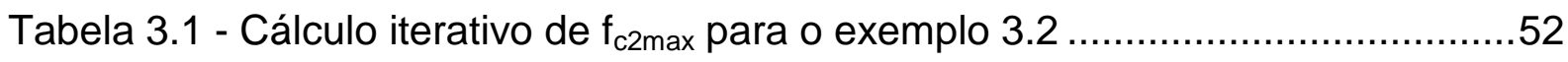

Tabela 3.2 - Cálculo iterativo de $\mathrm{f}_{\mathrm{c} 2 \mathrm{max}}$ para o exemplo 3.3 .................................55

Tabela 4.1 - Valores de $\theta_{1}, \theta_{2}$ e $\theta^{*}$ para os aços prescritos pela NBR $6118 \ldots \ldots \ldots \ldots . . .63$

Tabela 4.2 - Valores máximos para $\theta$ para o caso III .......................................67

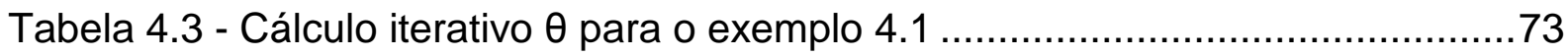

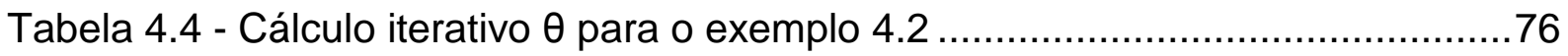

Tabela 4.5 - Valores de $\theta_{1}, \theta_{2}$ e $\theta^{*}$ para os aços prescritos pela NBR 6118 para o

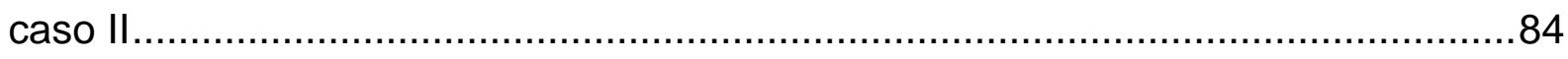

Tabela 4.6 - Valores máximos para $\theta$ para o caso II .......................................85

Tabela 6.1 - Esforços solicitantes equivalentes ao exemplo em 5.5 para vários ângulos $\varphi$ 


\section{LISTA DE ABREVIATURAS E SIGLAS}

$\mathrm{ACl} \quad$ American Concrete Institute

CEB Comité Euro-International du Béton

ELU Estado Limite Último

ELS Estado Limite de Serviço

NBR Norma Brasileira

MCFT Modified Compression Field Theory 


\section{LISTA DE SÍMBOLOS}

\section{SÍMBOLOS LATINOS}

\begin{tabular}{|c|c|}
\hline$a_{s x}$ & área de armadura na direção x \\
\hline $\mathrm{a}_{\mathrm{sy}}$ & área de armadura na direção y \\
\hline$E_{C S}$ & módulo de elasticidade do aço \\
\hline$f_{c}^{\prime}$ & resistência característica à compressão do concreto segundo o $\mathrm{ACl}$ \\
\hline $\mathrm{f}_{\mathrm{c} 2}$ & tensão no concreto na direção 2 \\
\hline$f_{c d}$ & resistência de cálculo à compressão do concreto \\
\hline$f_{c k}$ & resistência característica à compressão do concreto \\
\hline$f_{\mathrm{cd} 1}$ & resistência de cálculo à compressão para o concreto não-fissurado \\
\hline$f_{c d 2}$ & resistência de cálculo à compressão do concreto fissurado \\
\hline $\mathrm{f}_{\mathrm{c} 2 \mathrm{max}}$ & resistência máxima à compressão do concreto \\
\hline$f_{y d}$ & tensão de escoamento de cálculo do aço \\
\hline$f_{y k}$ & tensão de escoamento característica do aço \\
\hline$F_{x}$ & forças na direção x \\
\hline $\mathrm{F}_{\mathrm{y}}$ & forças na direção y \\
\hline $\mathrm{h}$ & espessura da chapa \\
\hline $\mathrm{n}_{\mathrm{c}}$ & maior esforço resistente de compressão no concreto \\
\hline$n_{c}^{\prime}$ & menor esforço resistente de compressão no concreto \\
\hline $\mathrm{n}_{\mathrm{x}}$ & esforço solicitante normal na direção x \\
\hline $\mathrm{n}_{\mathrm{y}}$ & esforço solicitante normal na direção y \\
\hline $\mathrm{n}_{\mathrm{xy}}$ & esforço de cisalhamento solicitante \\
\hline $\mathrm{n}_{\mathrm{sx}}$ & esforço resistente na armadura na direção x \\
\hline $\mathrm{n}_{\mathrm{sy}}$ & esforço resistente na armadura na direção y \\
\hline $\mathrm{R}_{\mathrm{d}}$ & resistência de cálculo \\
\hline$S_{d}$ & solicitação de cálculo \\
\hline
\end{tabular}




\section{SÍMBOLOS GREGOS}

\begin{tabular}{|c|c|}
\hline$Y_{x y}$ & distorção no plano xy \\
\hline$\varepsilon_{1}$ & deformação na direção 1 \\
\hline$\varepsilon_{2}$ & deformação na direção 2 \\
\hline$\varepsilon_{x}$ & deformação na direção $x$ \\
\hline$\varepsilon_{y}$ & deformação na direção y \\
\hline$\varepsilon_{\mathrm{sx}}$ & deformação da armadura na direção x \\
\hline$\varepsilon_{\text {sy }}$ & deformação da armadura na direção y \\
\hline$\varepsilon_{\mathrm{c}}$ & deformação do concreto \\
\hline$\varepsilon_{\mathrm{c} 1}$ & deformação de escoamento do concreto \\
\hline$\varepsilon_{\mathrm{cu}}$ & deformação última para o concreto \\
\hline$\varepsilon_{c u}^{*}$ & $\begin{array}{l}\text { deformação limite para considerar a resistência do concreto com } \\
\text { diagrama de tensão uniforme }\end{array}$ \\
\hline$\varepsilon_{\mathrm{c}}{ }^{\prime}$ & deformação correspondente ao tensão $f_{c}^{\prime}$ \\
\hline$\varepsilon_{y d}$ & deformação de escoamento de cálculo do aço \\
\hline$\theta$ & ângulo entre o eixo y e a direção principal de compressão no concreto \\
\hline$\theta_{1}$ & ângulo limite entre as curvas $f_{c d 1}$ e $f_{c 2 m a x}$ \\
\hline$\theta_{2}$ & ângulo limite entre as curvas $f_{c 2 m a x}$ e $f_{c d 2}$ \\
\hline$\theta^{*}$ & ângulo limite para que define $o$ sinal de $\varepsilon_{y}$ \\
\hline$\theta_{\max }$ & $\begin{array}{l}\text { ângulo limite para dimensionamento das armaduras de compressão no } \\
\text { caso II e III }\end{array}$ \\
\hline$\theta_{x y}$ & ângulo correspondente ao esforço de cisalhamento máximo \\
\hline$\theta_{c 1}$ & $\begin{array}{l}\text { ângulo limite para que a chapa permaneça em estado biaxial de } \\
\text { tensão no caso IV }\end{array}$ \\
\hline$\theta_{\mathrm{c} 2}$ & $\begin{array}{l}\text { ângulo limite para que a chapa permaneça em estado biaxial de } \\
\text { tensão no caso IV }\end{array}$ \\
\hline$\theta_{\mathrm{x}}$ & $\begin{array}{l}\text { ângulo limite para que a esforço na armadura na direção } x \text { seja de } \\
\text { compressão no caso IV }\end{array}$ \\
\hline$\theta_{\mathrm{y}}$ & $\begin{array}{l}\text { ângulo limite para que a esforço na armadura na direção y seja de } \\
\text { compressão no caso IV }\end{array}$ \\
\hline$\theta_{\text {amin }}$ & ângulo $\theta$ que resulta na menor armadura necessária para o caso IV \\
\hline$\sigma_{\mathrm{c}}$ & tensão no concreto na direção de maior compressão \\
\hline
\end{tabular}


$\sigma_{\mathrm{c}}{ }^{\prime} \quad$ tensão no concreto na direção de menor compressão

$\sigma_{\mathrm{cd}} \quad$ tensão de cálculo no concreto

$\varphi$

ângulo entre o eixo y e a direção principal de compressão dos esforços solicitantes 


\section{SUMÁRIO}

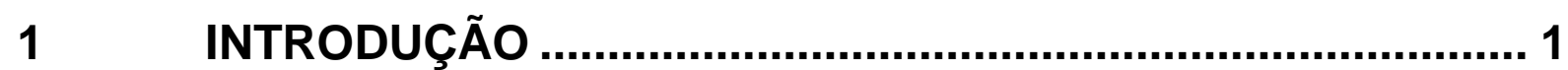

1.1 Conceituação e aplicação das chapas........................................ 1

1.2 Características básicas dos elementos de chapas ..................... 3

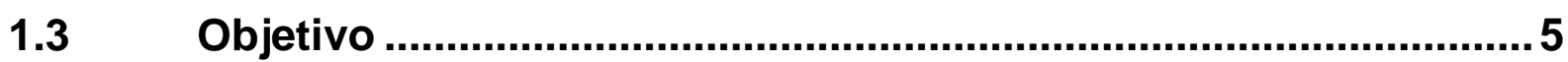

2 DIMENSIONAMENTO DE ELEMENTOS DE CHAPA ............. 7

2.1 Principais aspectos do comportamento resistente das chapas .7

2.2 Métodos de dimensionamento....................................................10

2.3 Método baseado nos critérios de Baumann ...............................15

2.3.1 Equações de equilíbrio...................................................................16

2.3.2 Equação de Compatibilidade ........................................................19

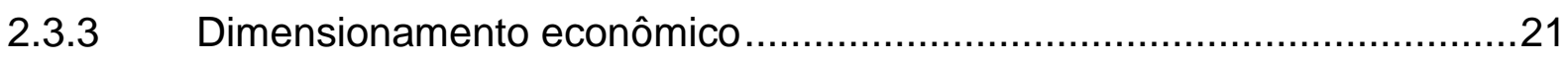

2.3.4 Resistência de projeto dos materiais …………................................25

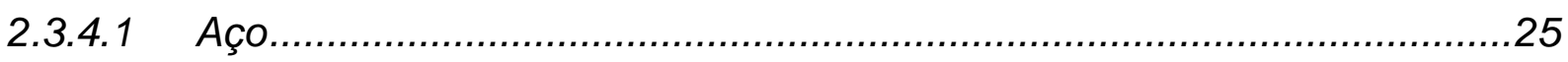

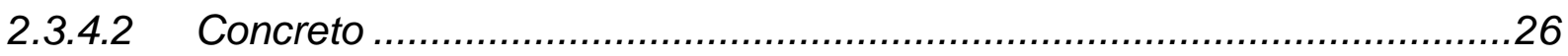

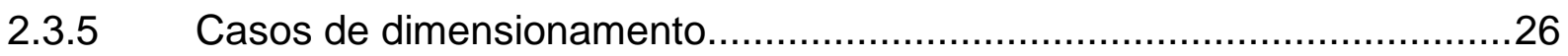

2.3.5.1 Caso I - Armaduras nas duas direções .............................................27

2.3.5.2 Caso II - Armadura apenas na direção y …........................................28

2.3.5.3 Caso III - Armadura apenas na direção x................................................30

2.3.5.4 Caso IV - Dispensa de armadura nas duas direções ...............................31

2.4 Considerações sobre o estudo das armaduras de compressão 34

3 MODELO RESISTENTE E VERIFICAÇÃO DA TENSÃO DE COMPRESSÃO NO CONCRETO .................................................... 36

3.1 Modelo resistente para o concreto em compressão ....................36 
3.2 Análise das deformações

3.3 Verificação da resistência à compressão do concreto

3.3.1 Cálculo para determinação do limite de resistência a compressão do concreto 45

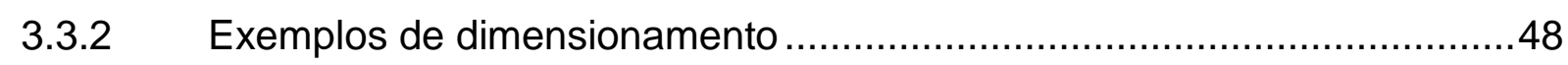

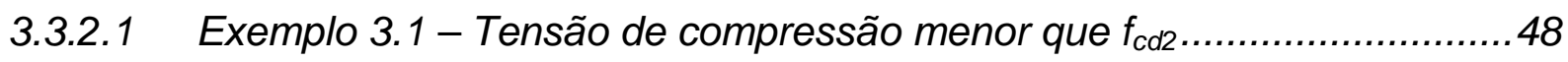

3.3.2.2 Exemplo 3.2 - Tensão de compressão entre $f_{c d 1}$ e $f_{c d 2}$ e abaixo de $f_{c 2 \max } 50$

3.3.2.3 Exemplo 3.3 - Tensão de compressão entre $f_{c d 1}$ e $f_{c d 2}$ e acima de $f_{c 2 m a x} . .53$

3.3.2.4 Exemplo 3.4 - Tensão de compressão maior que $f_{c d 1}$..............................55

4 DIMENSIONAMENTO DAS ARMADURAS DE COMPRESSÃO

PARA OS CASOS II E III ................................................................. 58

4.1 Hipóteses do problema .................................................................. 58

$4.2 \quad$ Limites de dimensionamento ......................................................59

4.3 Dimensionamento das armaduras..........................................67

4.4 Exemplos de dimensionamento .............................................70

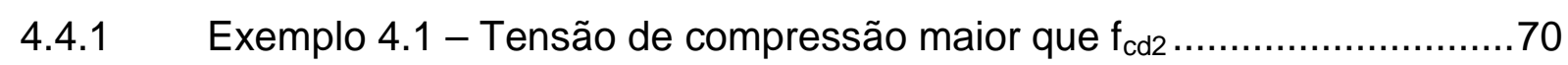

4.4.2 Exemplo 4.2 - Tensão de compressão igual a $f_{\mathrm{cd} 2}$................................75

4.4.3 Exemplo 4.3 - Tensão de cisalhamento maior que o limite .......................78

4.4.4 Exemplo 4.4 - Problema com solução inexistente..................................81

4.5 Dimensionamento das armaduras para o caso II........................ 83

5 DIMENSIONAMENTO DAS ARMADURAS DE COMPRESSÃO

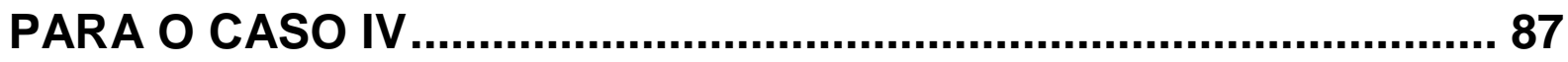

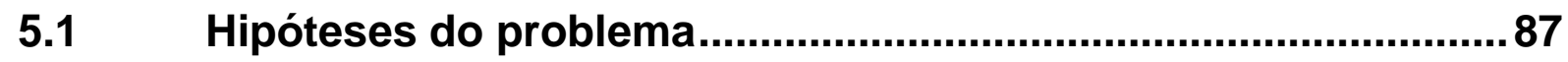

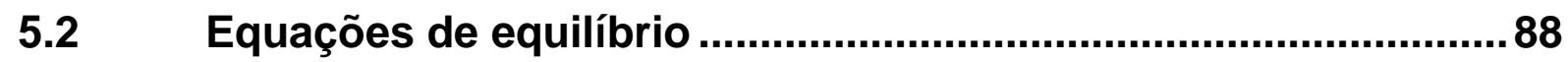

$5.3 \quad$ Limites de dimensionamento ........................................................ 91

5.4 Dimensionamento das armaduras...............................................94 


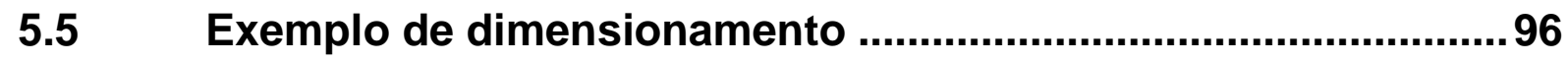

6 DISCUSSÃO DOS RESULTADOS .................................. 102

6.1 Casos II e III de dimensionamento............................................. 102

6.2 Caso IV de dimensionamento …..............................................107

$7 \quad$ CONCLUSÕES ................................................................... 112

REFERÊNCIAS BIBLIOGRÁFICAS .......................................... 114 


\section{INTRODUÇÃO}

\subsection{Conceituação e aplicação das chapas}

Em geral, pode-se reproduzir o comportamento das estruturas através de elementos básicos, normalmente classificados por sua forma geométrica e pelos esforços preponderantes sobre eles.

A norma NBR 6118 (2003) classifica estes elementos primeiramente em lineares ou de superfície. Os elementos lineares são aqueles em que uma das dimensões é relativamente maior que as outras duas como, por exemplo, vigas e pilares. Já os de superfície são aqueles em que uma das dimensões, usualmente chamada de espessura, é relativamente menor que as outras duas.

Os elementos de superfície, de acordo com a NBR 6118 (2003) são subdivididos em:

- Placas: Elementos de superfície plana sujeitos principalmente a ações normais ao seu plano, caso das lajes, por exemplo.

- Chapas: Elementos de superfície plana, sujeitos principalmente a ações contidas em seu plano.

- $\quad$ Cascas: Elementos de superfície não plana

- $\quad$ Pilares-parede: Elementos de superfície plana ou casca cilíndrica, dispostos usualmente na vertical e submetidos preponderantemente à compressão.

Nas chapas, tema deste trabalho, as ações atuantes produzem esforços solicitantes de membrana, isto é, esforços normais $n_{x}$ e $n_{y}$ e esforços de cisalhamento $n_{x y}$ contidos no plano do elemento, como apresentado na Figura 1.1. 


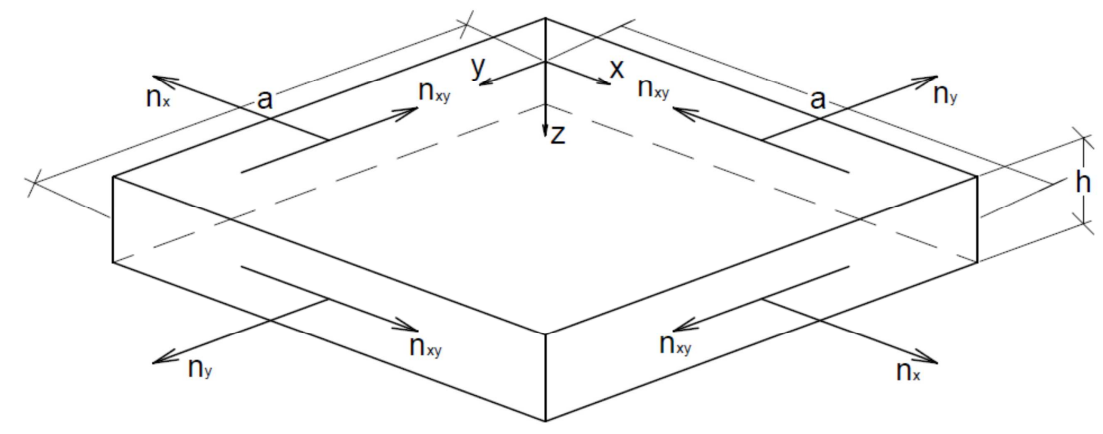

Figura 1.1 - Elemento de chapa

A importância do estudo deste tipo de elemento não se deve somente ao aprimoramento do entendimento e do dimensionamento de estruturas que são representadas por ele, mas também porque outros tipos de elementos podem ser decompostos em chapas.

Por exemplo, conforme o código modelo do Comité Euro-International du Betón (CEB, 1990), um elemento de placa pode ser representado por um modelo de três camadas sobrepostas, no qual as camadas superior e inferior funcionam como chapas, pois resistem a esforços de membrana enquanto que a camada intermediária resiste a esforços de cisalhamento perpendiculares ao seu plano. A Figura 1.2 ilustra este modelo.
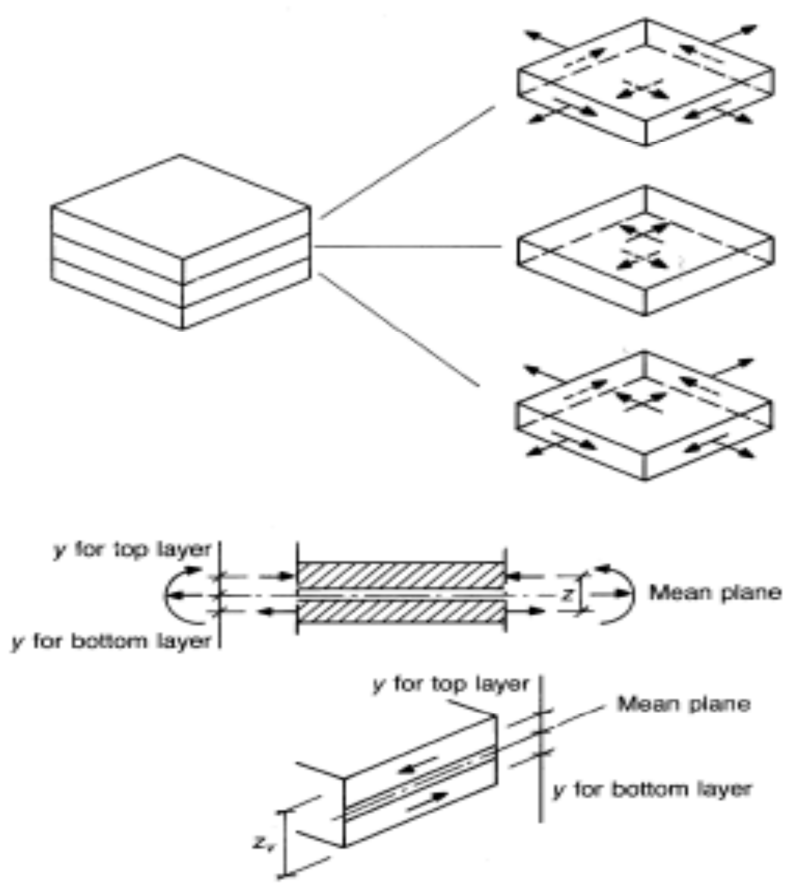

Figura 1.2 - Modelo de três camadas (CEB, 1990) 
Portanto, existe uma diversidade grande de estruturas em que as chapas podem ser aplicadas como elemento estrutural básico, podendo-se citar vigas-paredes, almas de vigas, silos, pontes celulares, reservatórios, base de plataformas marítimas, torres de resfriamento de usinas nucleares, entre outras. Alguns desses exemplos estão ilustrados na Figura 1.3. Assim, é importante o estudo das chapas de forma a desenvolver $\mathrm{o}$ entendimento do comportamento e o dimensionamento destas estruturas.

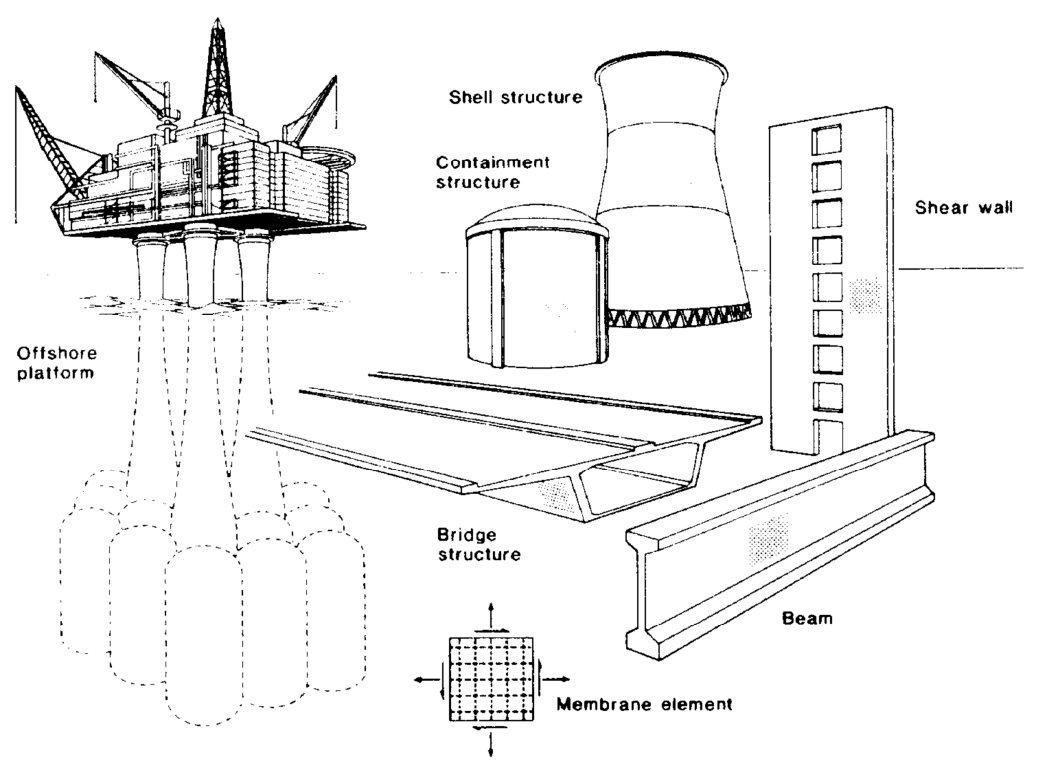

Figura 1.3 - Exemplos de utilização de elementos de chapas em estruturas

(VECCHIO; COLLINS, 1986)

\subsection{Características básicas dos elementos de chapas}

$\mathrm{O}$ concreto armado é composto por dois materiais distintos, o concreto e o aço. $\mathrm{O}$ concreto é um material que resiste bem à compressão, porém com baixa resistência à tração. Já o aço resiste de forma equivalente tanto à tração quanto à compressão. Portanto, nas peças de concreto armado, é desejável que somente as armaduras resistam aos esforços de tração.

Em qualquer estrutura de concreto armado, a melhor eficiência da armadura se dá quando ela é posicionada na direção da tensão principal de tração da peça, pois, 
assim, ela pode absorver diretamente este esforço. Entretanto, no caso das chapas, para cada combinação de carregamento existe uma direção principal de tração, sendo, portanto, raras as vezes em que é possível determinar um único posicionamento da armadura em que ela estaria na sua condição ótima.

Além disso, as estruturas são normalmente subdivididas em vários elementos de chapa e, assim, seria inadequado construtivamente que as armaduras fossem posicionadas em direções distintas para cada elemento.

Normalmente, elas são dispostas na estrutura seguindo um padrão. Este trabalho somente aborda os casos de posicionamento ortogonal da armadura, porque é o caso mais comum e construtivamente mais simples, mas existem outros padrões de posicionamento que são mais adequados a casos específicos, conforme exemplificado na Figura 1.4.

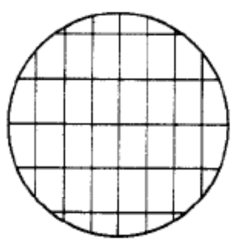

(a) Orthogonal Grid

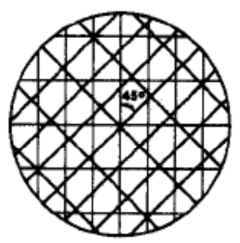

(c) Two Orthogonal Grids

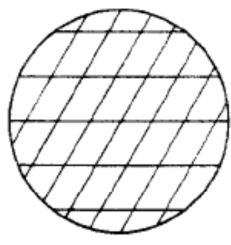

(b) Skew Grid

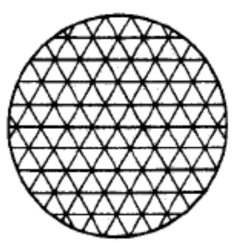

(d) Triangle Grid

Figura 1.4 - Padrões de posicionamento para armaduras (GUPTA, 1984)

Assim, na maioria dos casos, a direção da armadura não corresponderá à direção das tensões principais de tração atuantes no elemento.

Dado este quadro, surgem duas questões principais. Como dimensionar este elemento de forma a evitar sua ruptura e como prever seu comportamento de forma a verificar as condições da estrutura em serviço.

Devido aos aspectos descritos, o dimensionamento para o Estado Limite Último (ELU), ou seja, a quantificação das armaduras e a verificação da tensão de compressão no concreto nas chapas, não é tarefa trivial. Apesar de complexo, este 
problema já foi objeto de estudo de vários pesquisadores e há alguns métodos de resolução. Uma das primeiras soluções foi dada por Baumann, em 1972 ${ }^{1}$. Ele supõe algumas hipóteses que tornam o seu modelo um dos mais simples de se operar.

As soluções propostas para o ELU normalmente abordam casos em que a armadura trabalha tracionada. Outra questão que surge a respeito do dimensionamento de chapas se refere ao caso em que a tensão de compressão atuante no concreto é maior que sua tensão limite. As soluções possíveis deste problema passam pelo aumento da resistência característica do concreto, aumento da sua espessura ou adoção de armaduras que ajudem a combater o esforço de compressão.

No cálculo de armaduras para a compressão estão presentes problemas semelhantes ao dimensionamento para a tração. Porém, para este caso não se encontram critérios de dimensionamento na literatura.

Outra abordagem para o tema é a previsão de comportamento destes elementos, ou seja, dimensionamento para o Estado Limite de Serviço (ELS). Este problema é bastante complexo devido aos vários fenômenos que ocorrem quando a chapa é submetida a um carregamento gradual. Estes fenômenos serão apresentados posteriormente.

\subsection{Objetivo}

Nos casos em que o concreto não satisfaz a condição de que sua capacidade resistente à compressão seja maior que a tensão solicitante, uma solução possível seria introduzir armadura submetidas à compressão para que parte da tensão seja absorvida por elas. Conforme apresentado, são muito escassos estudos que abordam este problema.

Existem vários métodos analíticos para obter o dimensionamento das armaduras em chapas, porém estas soluções normalmente estão voltadas para problemas em que as armaduras estão submetidas apenas a tração,

Este trabalho tem como objetivo obter critérios de utilização e dimensionamento para o ELU de chapas com armaduras dispostas ortogonalmente com pelo menos uma

\footnotetext{
${ }^{1}$ BAUMANN, T. Zur Frage der Netzbewehrung von Flachentragwerken. Der Bauingenieur, Vol. 47, № 10, 1972, p.367-377
} 
das direções das armaduras submetida à compressão, tendo como fundamento o método baseado nos critérios de Baumann (1972). 


\section{DIMENSIONAMENTO DE ELEMENTOS DE CHAPA}

\subsection{Principais aspectos do comportamento resistente das chapas}

O comportamento de um elemento de chapa é regido por vários fenômenos que atuam simultaneamente.

Basicamente, no início da aplicação de um carregamento gradual que introduza tração em uma das direções principais de tensão, o concreto irá resistir a esta tração e não há fissuração. Nesta situação, a peça se comporta como um material homogêneo submetido a um estado plano de tensão. $O$ aço tem pouca influência nesta fase porque a maior parte dos esforços é resistida pelo concreto.

Após a tensão de tração superar a resistência do concreto, a armação começa a ser solicitada e surgem as primeiras fissuras perpendiculares à direção principais dos esforços solicitantes de tração.

Conforme o carregamento aumenta, as fissuras iniciais diminuem ou se fecham e surgem novas fissuras em outra direção. Isto ocorre devido à introdução de esforços causados pela armadura e também pela tensão de cisalhamento que aparece entre as fissuras, provocada principalmente por dois fenômenos, o engrenamento dos agregados e o efeito de pino.

O efeito de pino consiste na transferência de esforços de cisalhamento provocado pelo encavilhamento da armadura, como mostrado na Figura 2.1.

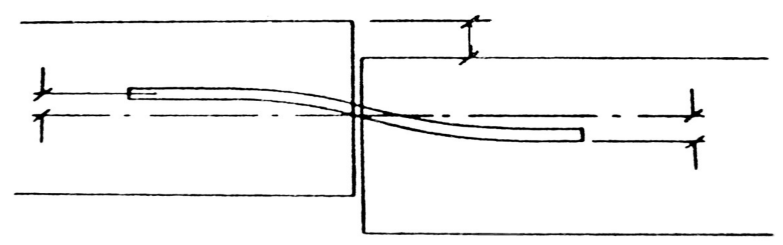

Figura 2.1 - Efeito de pino (adaptado do CEB, 1990)

Já o engrenamento dos agregados ocorre porque os concretos de resistência usuais, diferentemente dos de alta resistência, fissuram normalmente nas regiões de 
pasta de cimento. Isto permite que a interface da fissura seja, em geral, bastante rugosa, pois o agregado graúdo fica exposto conforme ilustrado pela Figura 2.2.

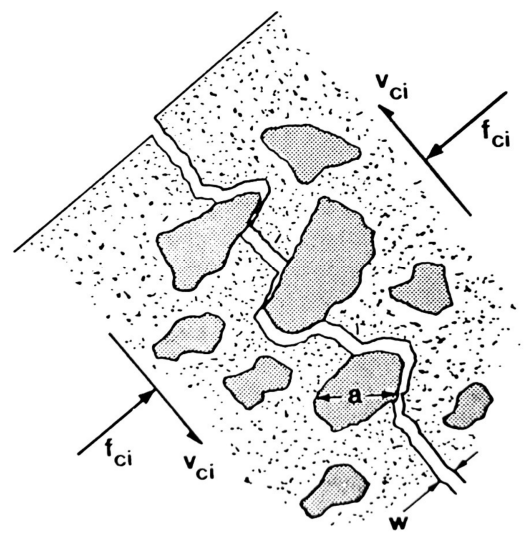

Figura 2.2 - Engrenamento dos agregados (VECCHIO E COLLINS, 1986)

A chapa de concreto também fica sujeita a outro fenômeno chamado "tensionstiffening". Na região entre fissuras, a armadura tracionada transmite por meio de forças de ligação uma parte desta tração para o concreto. A contribuição para a resistência à tração da chapa oferecida pelo concreto provoca um aumento de rigidez do elemento (CEB, 1990). A Figura 2.3 mostra um gráfico no qual se pode ver que a armadura isolada apresenta menos rigidez que na situação em que está trabalhando juntamente com o concreto.

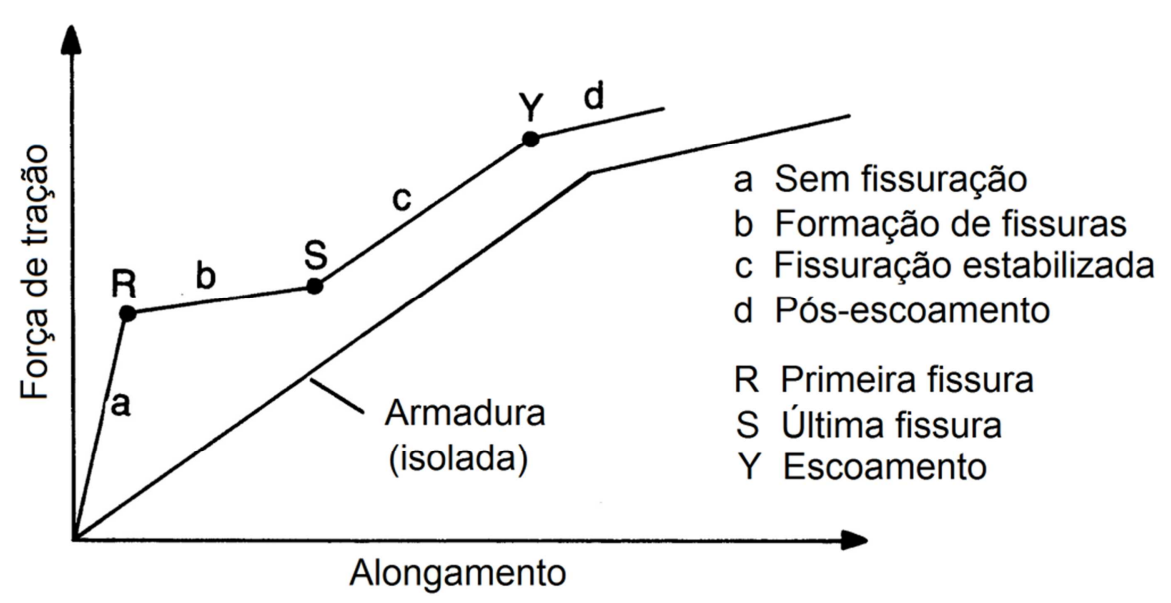

Figura 2.3 - Acréscimo de rigidez devido ao efeito do "tension-stiffening" (adaptado CEB, 1990)

Outro fenômeno importante a ser considerado é o amolecimento do concreto fissurado. Nos casos em que o concreto está fissurado, sua rigidez e sua resistência 
à compressão são menores quando comparado ao concreto intacto. A Figura 2.4 ilustra este fenômeno.

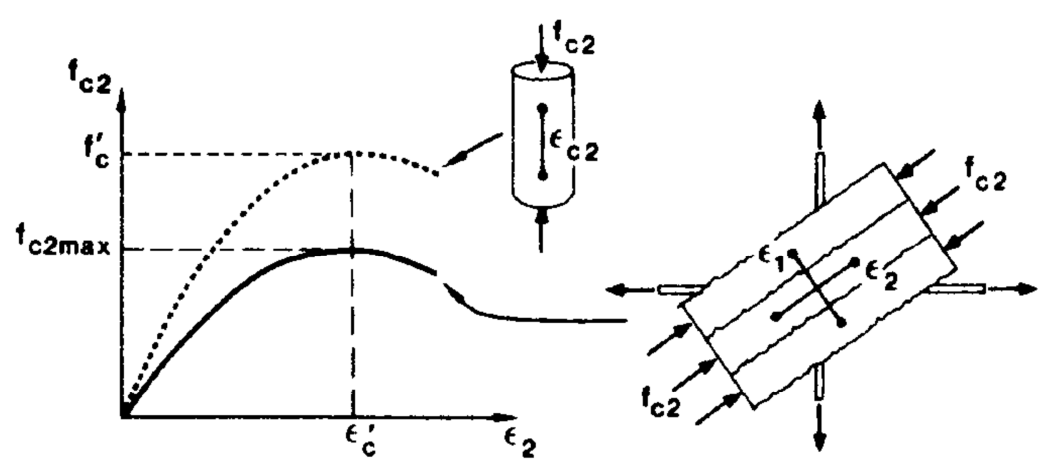

Figura 2.4 - Amolecimento do concreto fissurado (VECCHIO E COLLINS, 1986)

Assim, tem-se o quadro dos principais fenômenos que influenciam o comportamento da chapa quando há tração em pelo menos uma das direções principais, podendose notar que não é tarefa trivial a sua modelagem.

Se a chapa for submetida a esforços apenas de compressão a situação se modifica porque neste caso não há fissuração do concreto. Nesta situação, há duas possibilidades para dimensionamento. A primeira em que se pode dispensar o uso de armaduras de compressão, pois a tensão solicitante no concreto está abaixo da sua resistência, e a segunda em que se devem introduzir armaduras que auxiliem o combate à compressão.

Em ambos os casos é possível considerar um acréscimo de resistência do concreto, pois a chapa está em estado biaxial de compressão. A Figura 2.5 mostra o gráfico que representa o modelo proposto por Kupfer et al. (1969) ${ }^{2}$ apud CEB (1990) para a resistência do concreto em estado biaxial de tensão.

\footnotetext{
${ }^{2}$ KUPFER, H.; GERSTLE, K. H.; RÜSCH, H. Behaviour of concrete under biaxial stress. Proc. ACl, v. 66, August 1969. p. 656-666.
} 


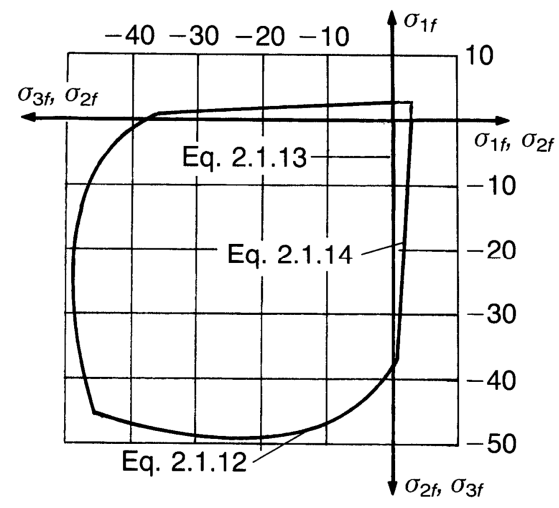

Figura 2.5 - Modelo para resistência biaxial do concreto C30 (KUPFER et al. ,1969 apud CEB, 1990)

Quanto ao seu comportamento, é possível idealizá-lo como aquele obtido por um material homogêneo submetido a estado plano de tensão. Porém, quando há adoção de armaduras, as equações devem considerar esse esforço resistente.

\subsection{Métodos de dimensionamento}

Vários pesquisadores se dedicaram ao desenvolvimento de métodos de dimensionamento para chapas propondo hipóteses e simplificações do problema de modo a obter uma solução coerente e a favor da segurança. Citar-se-á alguns dos mais importantes e suas contribuições para o desenvolvimento do estudo deste problema.

Conforme citado por Leonhardt e Mönning (1978) e Gupta (1984), Leitz (1923) foi um dos primeiros a abordar este assunto. Ele considerou que as fissuras eram perpendiculares às armaduras e equacionou as condições de equilíbrio. Outros trabalhos se seguiram ao dele.

Peter $(1964)^{2}$ realizou um experimento que consistia em submeter nove painéis com armaduras ortogonais em diferentes ângulos a carregamento de tração uniaxial. A princípio, ele considerou que as fissuras sempre eram perpendiculares ao carregamento uniaxial e propôs uma formulação baseado nesta hipótese. Porém, ele percebeu que sua teoria não correspondia com a realidade, pois dois dos seus

\footnotetext{
${ }^{3}$ LEITZ, H. Eisenbewehrt Platen bei Allgemeinem Biegungzustand. Die Bautechnik 1, №16, p.155157, №17, p.163-167, 1923.
} 
painéis, que estavam com taxas de armadura diferentes nas duas direções, não apresentavam resultados condizentes.

Após isso, Nielsen $(1971)^{4}$ propõe um modelo baseado no conceito de membrana fissurada, que consiste em supor que a membrana é um meio contínuo, onde as armaduras resistem apenas a esforços axiais e o concreto é submetido a esforço uniaxial, conforme a Figura 2.6.

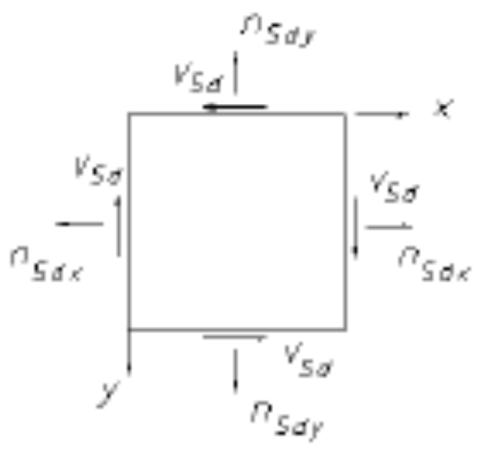

(a)

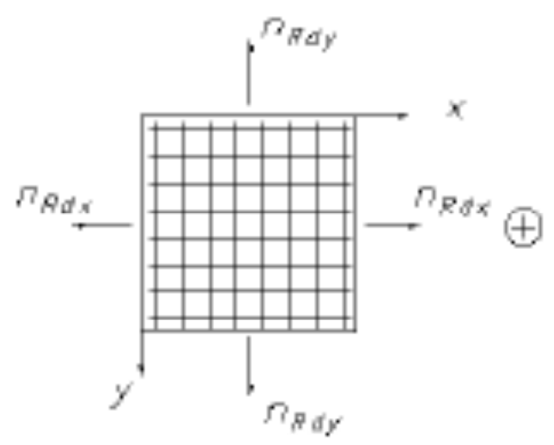

(b)

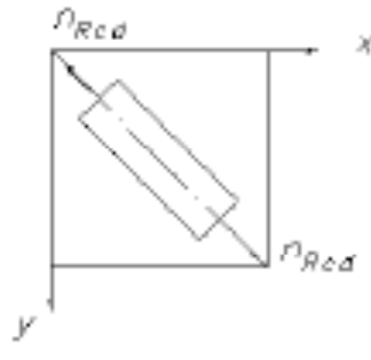

(c)

Figura 2.6 - Modelo para elemento de membrana proposto por Nielsen: (a) esforções solicitantes; (b) contribuição da armadura; (c) contribuição do concreto (CHEN, 2004)

Baumann (1972) ${ }^{1}$ elabora equações que satisfazem tanto o equilíbrio quanto a compatibilidade da chapa (LEONHARDT E MÖNNING, 1978). Este modelo será apresentado com detalhes posteriormente, mas ele se baseia na premissa que não há tensão de cisalhamento ao longo das fissuras. As soluções propostas por Baumann e por Nielsen chegam aos mesmos resultados, porém deduzidas a partir de modelos diferentes.

Gupta (1981) utiliza os critérios de Baumann para obter equações que permitem a verificação quanto ao ELU. Além disso, solucionou o problema de forma a obter a mínima taxa de armadura e a mínima compressão no concreto.

Vecchio e Collins $(1982)^{5}$ realizaram um experimento no qual trinta painéis de concreto armado com taxas de armadura diferentes nas duas direções foram

\footnotetext{
${ }^{4}$ M. P. NIELSEN, On the strenght of reinforced concrete discs, ACTA Polytechnica Scandinacia, Civil Engineering and Building Construction Series, № 10, Copenhagen, 1971

${ }^{5}$ VECCHIO, F. J.;COLLINS M. P. Response of Reinforced Concrete to In-Plane Shear and Normal Stresses. Publication № 82-03, Department of Civil Engineering, University of Toronto, March, 1982, 332p.
} 
submetidos a vários tipos de carregamento no plano. Este experimento está representado pela Figura 2.7 .

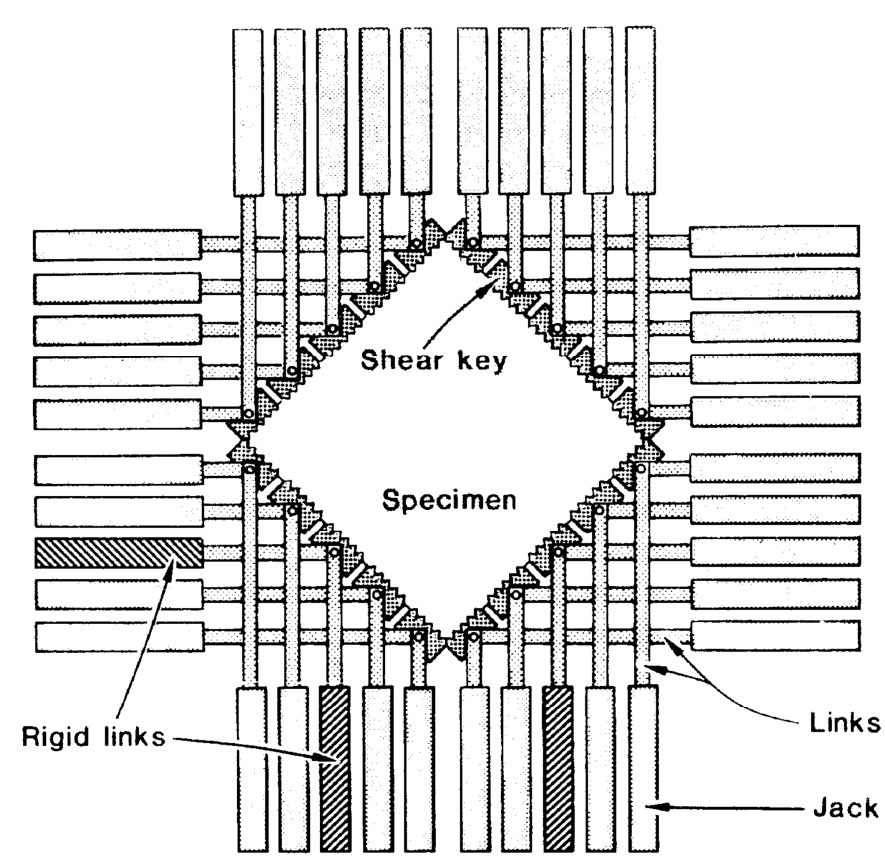

Figura 2.7 - Experimento de Vecchio e Collins (1982)

Fialkow (1983) adapta os critérios propostos no Building Code ACl 318-77 do American Concrete Institute $(\mathrm{ACl})$ para dimensionamento de elementos lineares aos elementos de chapa, levando em consideração não somente a resistência axial da armadura e a resistência à compressão do concreto, mas também a resistência ao cisalhamento tanto oferecida pela armadura quanto pelo concreto.

Gupta e Akbar (1984), baseado nos experimentos de Peter (1964) ${ }^{1}$ e de Vecchio e Collins $(1982)^{6}$, apresentam um modelo com objetivo de não somente dimensionar a chapa, mas também prever seu comportamento quando submetida a um carregamento gradual. A resposta ao carregamento é distinta dependendo de qual etapa o elemento se encontra, sendo possível distinguir quatro etapas.

- $\quad 1^{\circ}$ etapa: o concreto não está fissurado e as armaduras têm comportamento elástico

- $\quad 2$ e etapa: o concreto está fissurado e as armaduras nas duas direções têm comportamento elástico

- $\quad 3^{\circ}$ etapa: o concreto está fissurado e as armaduras de uma direção escoam. 
- $\quad 4^{0}$ etapa: o concreto está fissurado e as armaduras das duas direções escoam.

Na primeira etapa o elemento apresenta comportamento de um meio continuo em estado plano de tensão. A última etapa se refere ao estado limite último da peça, problema para o qual já havia algumas soluções. Gupta e Akbar (1984) apresentam soluções que permitem prever o comportamento da chapa para as etapas intermediárias. Para tanto, utilizam algumas hipóteses simplificadoras do problema como a não existência de tensão de cisalhamento entre as fissuras. Eles mencionam o conceito de rotação das fissuras, no qual a direção das fissuras muda conforme o carregamento aumenta.

Vecchio e Collins (1986) propõem o Modified Compression Field Theory (MCFT). Este modelo leva em conta o efeito de "tension-stiffening", pressupõe a existência de tensão de cisalhamento na fissura, mas transmitida apenas pelo engrenamento dos agregados e considera também o amolecimento do concreto fissurado. Como tenta se aproximar melhor da realidade introduzindo mais variáveis, é, por consequência, mais complexo, mas alcança resultados satisfatórios. A Figura 2.8 ilustra as considerações do MCFT. 


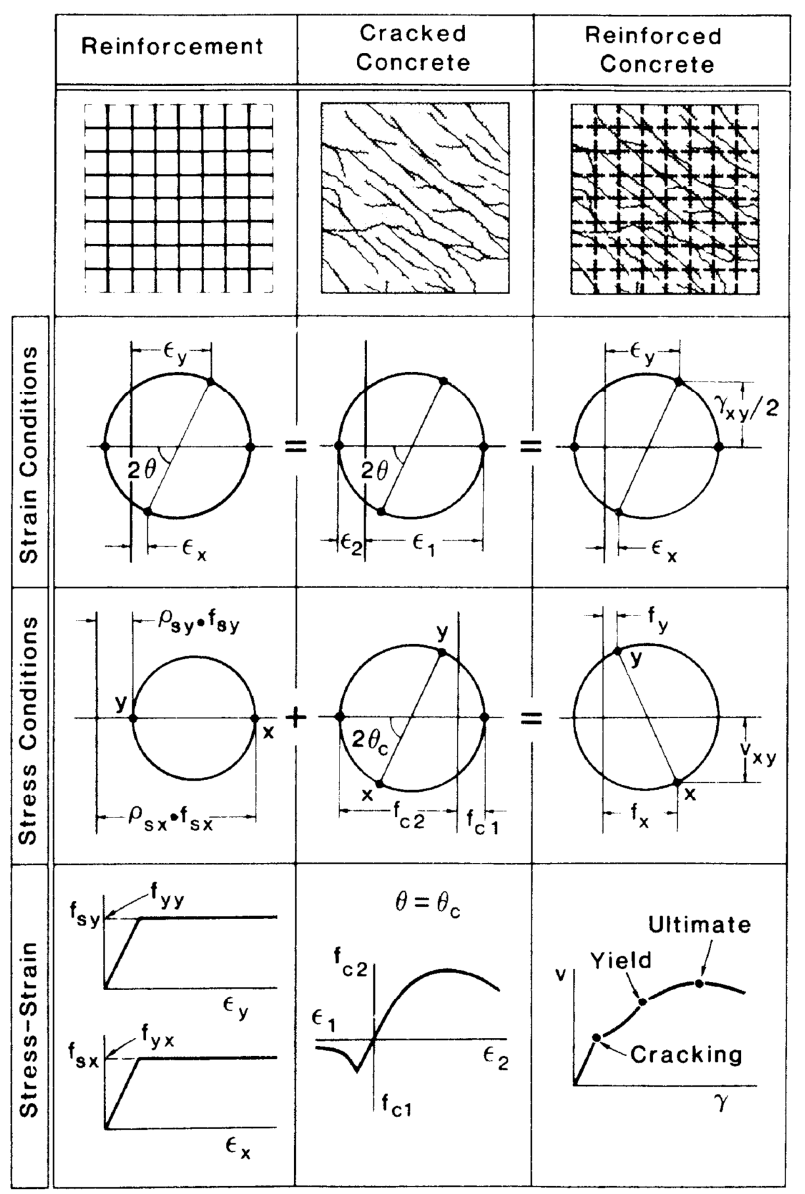

Figura 2.8 - Quadro resumo do MCFT (VECCHIO E COLLINS, 1986)

Chen (2004) compila em seu trabalho alguns dos métodos de dimensionamento supracitados, como aquele baseado nos critérios propostos por Baumann $(1972)^{5}$, Nielsen $(1971)^{4}$ e o elaborado por Fialkow (1983).

Jazra (2008) compara o MCFT com o método baseado nos critérios de Baumann, além de apresentar algumas formulações para dimensionamento de armaduras de compressão para chapas.

Estes trabalhos apresentados abordam soluções analíticas para o problema, que é tema deste trabalho. Porém, com o aumento da capacidade e da facilidade de utilização dos computadores, foram desenvolvidos vários métodos numéricos para modelagem de estruturas em geral e, consequentemente, aprimorou-se a modelagem das chapas.

Entre os métodos numéricos, o mais utilizado hoje é o método dos elementos finitos (MEF). Os softwares baseados neste método são muito difundidos hoje em dia, sendo ferramentas importantes da análise de estruturas. Quando se utilizam estes programas, normalmente é feita a análise linear da estrutura, admitindo 
comportamento elástico linear dos materiais, de forma a obter o campo de tensões atuantes na peça. A partir disto, se faz o dimensionamento das armaduras ou por métodos analíticos, como os citados anteriormente, ou por métodos numéricos.

Há também programas que fazem a análise não linear das estruturas, ou seja, levam em consideração a não linearidade física dos materiais. Assim, podem prever o comportamento da estrutura de forma mais precisa Entretanto, estes programas necessitam de muitos dados iniciais como a quantidade de barras e seu posicionamento, além de uma série de dados de entrada sobre os materiais utilizados, sendo normalmente empregados para verificações de peças especiais ou estudos de comportamento.

Os métodos numéricos não fazem parte do escopo desta dissertação.

\subsection{Método baseado nos critérios de Baumann}

O método de dimensionamento baseado nos critérios de Baumann é, provavelmente, o mais simples de ser utilizado em dimensionamento de chapas. Por essa razão, ele foi escolhido para ser a base desse estudo.

Este método em si não apresenta solução para o caso de adoção de armaduras de compressão, mas apoiado nele será proposta uma formulação e critérios de utilização destas armaduras.

Jazra (2008) compara este método com o MCFT proposto por Vecchio e Collins (1986) e chega a resultados que mostram que, comparativamente, o dimensionamento obtido é a favor da segurança. Esta conclusão era esperada, pois a formulação adota algumas hipóteses admitindo este resultado.

Porém, apesar de adequado para dimensionamento para o ELU, ele não obterá a mesma eficácia em prever características para verificação do ELS, como deformação e fissuração.

Ele tem como hipóteses básicas:

1. As fissuras apresentadas pelo elemento são aproximadamente paralelas e retilíneas.

2. A resistência à tração do concreto é desprezada. 
3. O efeito de pino das armaduras é desprezado.

4. O efeito de engrenamento dos agregados é desprezado.

5. É considerada perfeita aderência entre a armadura e o concreto.

6. É desconsiderado o efeito devido ao "tension-stiffening".

7. As direções das deformações principais coincidem com as direções das tensões principais.

\subsubsection{Equações de equilíbrio}

Considera-se um elemento de chapa submetido a esforços normais por unidade de comprimento $n_{x}$ e $n_{y}$ e ao esforço de cisalhamento, também por unidade de comprimento, $\mathrm{n}_{\mathrm{xy}}$, em que as armaduras estão posicionadas na direção dos eixos $\mathrm{x} e$ y. $O$ ângulo $\theta$ é aquele formado entre a direção principal de compressão no concreto com a direção y. Nos casos em que houver fissuração da chapa, este ângulo se refere também àquele formado pelo eixo y e a fissura, pois, como por hipótese, não há tensão de cisalhamento entre as fissuras, a direção principal de compressão no concreto, eixo 2, é paralela à fissura. Por hipótese, se considera que as direções principais de deformação e de tensão no concreto são coincidentes. Esta composição é ilustrada na Figura 2.9.

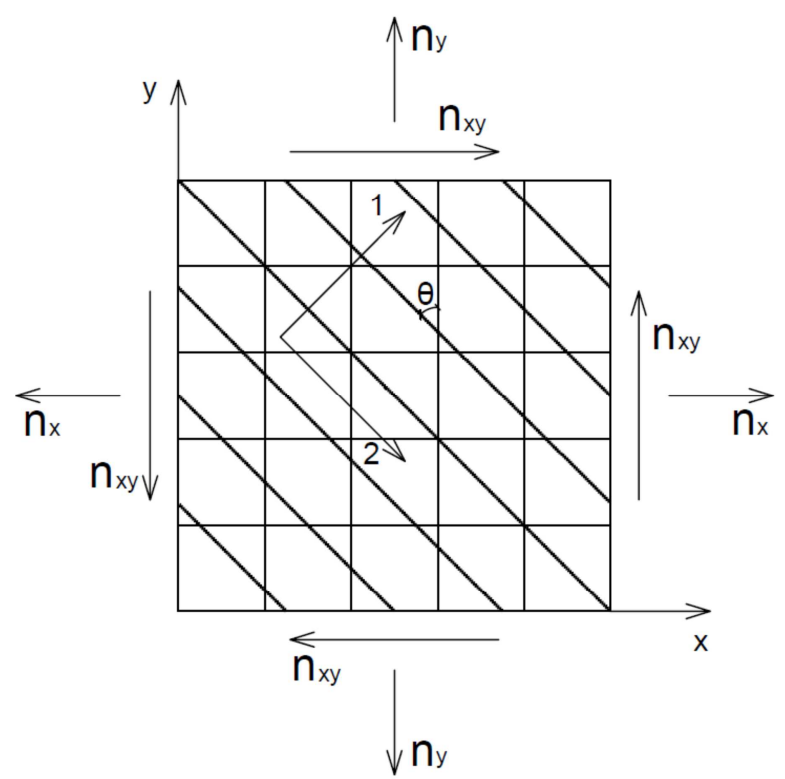

Figura 2.9 - Eixos considerados neste estudo 
O problema consiste em, conhecendo os esforços $n_{x}, n_{y}$ e $n_{x y}$, dimensionar as armaduras e verificar se o concreto resiste à tensão de compressão atuante. $A$ princípio, será considerado que as armaduras atuam somente a tração e, nos casos em que houver compressão em uma direção da armadura, se admitirá que ela não vá existir nessa dada direção.

A demonstração apresentada neste trabalho também é encontrada nos trabalhos de Leonhardt e Mönning (1978), Chen (2004) e Jazra (2008).

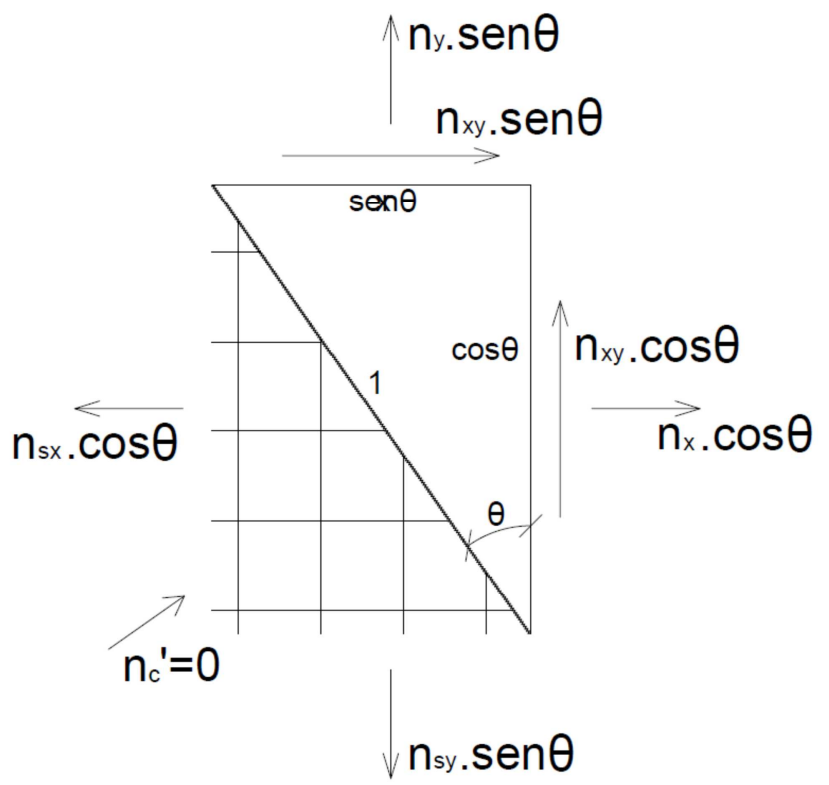

Figura 2.10 - Equilíbrio de forças na seção paralela à fissura

Primeiramente, admite-se uma seção paralela à fissura de comprimento unitário, como mostrado na Figura 2.10. Os esforços solicitantes $n_{x}, n_{y}$ no sentido de provocar esforços de tração e $n_{x y}$ no sentido mostrado na Figura 2.10 são convencionados positivos para todo o estudo.

Para esta seção, os esforços solicitantes são equilibrados pelos esforços resistentes $n_{\mathrm{sx}}, \mathrm{n}_{\mathrm{sy}}$, oferecidos pela armadura, e $\mathrm{n}_{\mathrm{c}}$ ' que representa o esforço de compressão no concreto na direção 1 . Neste caso, $n_{c}^{\prime}$ vale zero, pois na direção perpendicular à fissura o concreto está tracionado e, por hipótese, a resistência à tração do concreto é nula.

É convencionado positivo $n_{s x}$ e $n_{s y}$ quando de tração e $n_{c}$ e $n_{c}^{\prime}$ quando de compressão, sendo $\mathrm{n}_{\mathrm{c}} \mathrm{O}$ esforço de compressão na direção 2 .

A partir da Figura 2.10 podem-se estabelecer as relações de equilíbrio na direção $\mathrm{x}$, conforme eq. 2.1, e na direção y, conforme eq. 2.2. 
$\sum F_{x}=n_{x} \cdot \cos \theta+n_{x y} \cdot \operatorname{sen} \theta-n_{s x} \cdot \cos \theta=0$

$n_{s x}=n_{x}+n_{x y} \cdot \operatorname{tg} \theta$

$\sum F_{y}=n_{y} \cdot \operatorname{sen} \theta+n_{x y} \cdot \cos \theta-n_{s y} \cdot \operatorname{sen} \theta=0$

$n_{s y}=n_{y}+n_{x y} \cdot \operatorname{cotg} \theta$

Considerando agora outra seção, perpendicular à fissura, mostrada na Figura 2.11, pode-se estabelecer a equação 2.3 de equilíbrio.

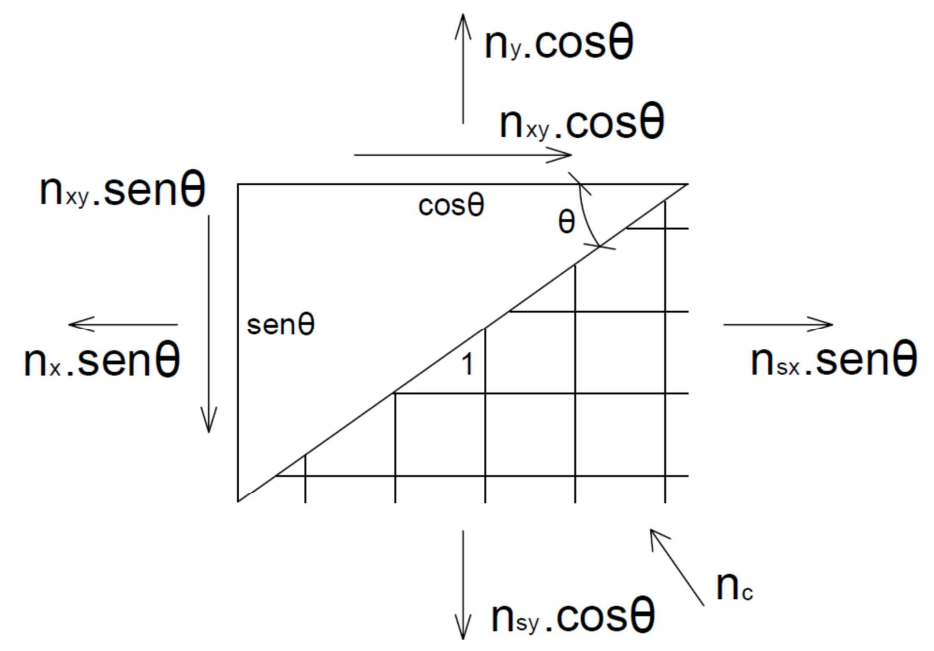

Figura 2.11 - Equilíbrio de forças na seção perpendicular à fissura

$\sum F_{x}=0$

$n_{c}-n_{s x} \cdot \operatorname{sen} \theta \cdot \operatorname{sen} \theta-n_{s y} \cdot \cos \theta \cdot \cos \theta+n_{x} \cdot \operatorname{sen} \theta \cdot \operatorname{sen} \theta-n_{x y} \cdot \operatorname{sen} \theta \cdot \cos \theta+$

$n_{S d y} \cdot \cos \theta \cdot \cos \theta-n_{x y} \cdot \cos \theta \cdot \operatorname{sen} \theta=0$

$n_{c}-n_{s x} \cdot \operatorname{sen}^{2} \theta-n_{s y} \cdot \cos ^{2} \theta+n_{x} \cdot \operatorname{sen}^{2} \theta-2 \cdot n_{x y} \cdot \operatorname{sen} \theta \cdot \cos \theta+n_{y} \cdot \cos ^{2} \theta=0$

Substituindo (2.1) e (2.2) em (2.3):

$$
\begin{gathered}
n_{c}-\left(n_{x}+n_{x y} \cdot \operatorname{tg} \theta\right) \cdot \operatorname{sen}^{2} \theta-\left(n_{y}+n_{x y} \cdot \operatorname{cotg} \theta\right) \cdot \cos ^{2} \theta+n_{x} \cdot \operatorname{sen}^{2} \theta-2 \cdot n_{x y} \cdot \operatorname{sen} \theta \cdot \cos \theta \\
+n_{y} \cdot \cos ^{2} \theta=0
\end{gathered}
$$




$$
\begin{aligned}
& \begin{array}{l}
n_{c}-n_{x} \cdot \operatorname{sen}^{2} \theta-n_{x y} \cdot \operatorname{tg} \theta \cdot \operatorname{sen}^{2} \theta-n_{y} \cdot \cos ^{2} \theta-n_{x y} \cdot \operatorname{cotg} \theta \cdot \cos ^{2} \theta+n_{x} \cdot \operatorname{sen}^{2} \theta \\
\quad-2 n_{x y} \cdot \operatorname{sen} \theta \cdot \cos \theta+n_{y} \cdot \cos ^{2} \theta=0
\end{array} \\
& n_{c}-n_{x y} \cdot \frac{\operatorname{sen} \theta}{\cos \theta} \cdot \operatorname{sen}^{2} \theta-n_{x y} \cdot \frac{\cos \theta}{\operatorname{sen} \theta} \cdot \cos ^{2} \theta-2 n_{x y} \cdot \operatorname{sen} \theta \cdot \cos \theta=0 \\
& n_{c}=n_{x y} \cdot\left[\operatorname{sen} \theta \cdot\left(\frac{\operatorname{sen}^{2} \theta}{\cos \theta}+\cos \theta\right)+\cos \theta \cdot\left(\frac{\cos ^{2} \theta}{\operatorname{sen} \theta}+\operatorname{sen} \theta\right)\right] \\
& n_{c}=n_{x y} \cdot\left[\operatorname{sen} \theta \cdot\left(\frac{\operatorname{sen}^{2} \theta+\cos ^{2} \theta}{\cos \theta}\right)+\cos \theta \cdot\left(\frac{\cos ^{2} \theta+\operatorname{sen}^{2} \theta}{\operatorname{sen} \theta}\right)\right] \\
& n_{c}=n_{x y} \cdot\left[\operatorname{sen} \theta \cdot\left(\frac{1}{\cos \theta}\right)+\cos \theta \cdot\left(\frac{1}{\operatorname{sen} \theta}\right)\right] \\
& n_{c}=n_{x y} \cdot(\operatorname{tg} \theta+\operatorname{cotg} \theta)
\end{aligned}
$$

Assim, o problema apresenta quatro incógnitas, sendo elas $n_{c}, n_{s x}, n_{s y}$ e $\theta$ e três equações de equilíbrio.

$$
\begin{aligned}
& n_{s x}=n_{x}+n_{x y} \cdot \operatorname{tg} \theta \\
& n_{s y}=n_{y}+n_{x y} \cdot \operatorname{cotg} \theta \\
& n_{c}=n_{x y} \cdot(\operatorname{tg} \theta+\operatorname{cotg} \theta)
\end{aligned}
$$

Se arbitrarmos qualquer uma das quatro incógnitas o problema fica determinado. Como os objetivos do problema são encontrar os esforços nas armaduras e no concreto, $\theta$ seria a variável livre.

No entanto, é desejável que o valor de $\theta$ a ser escolhido conduza a solução mais econômica, ou seja, que fornece a menor quantidade de armadura. Para se determinar esse valor, será utilizada mais uma equação que considera a compatibilidade de deformações do elemento.

\subsubsection{Equação de Compatibilidade}

Considerando uma seção paralela à fissura, conforme mostrada na Figura 2.12, a configuração deformada do elemento é representada pelo triangulo $A^{\prime}, B^{\prime}$ e $C^{\prime}$. O segmento de reta $A B$ foi comprimido, pois está na direção da fissura, ou seja, na 
região onde apenas o concreto está resistindo as tensões de compressão. Já os segmentos BC e AC estão tracionados, pois estão na direção das armaduras.

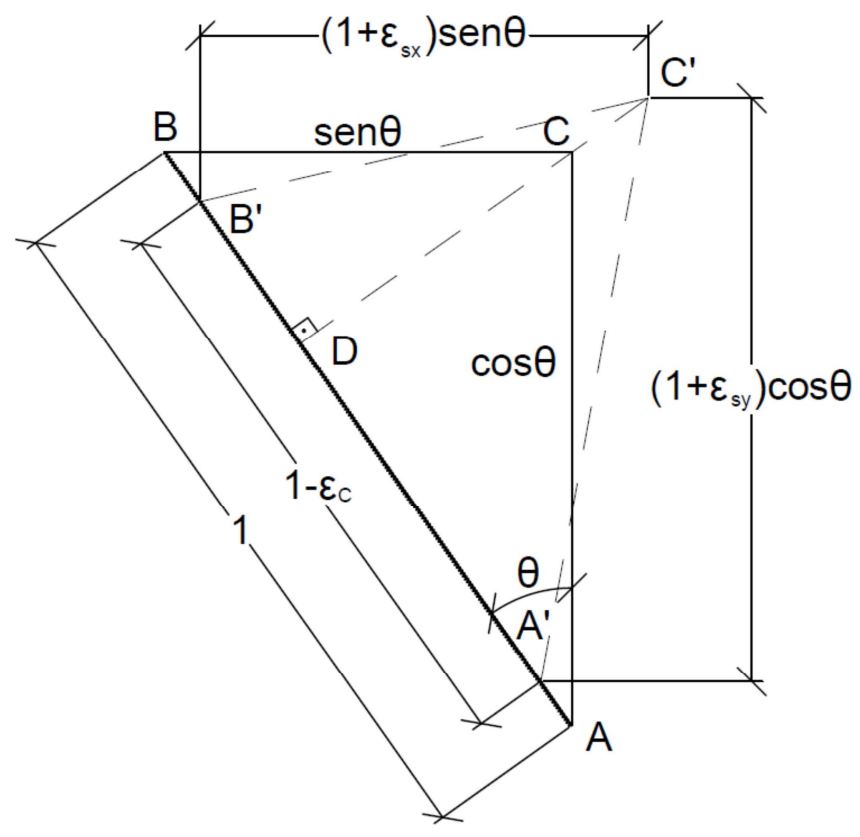

Figura 2.12 - Deformação da seção paralela a fissura

Desta forma, admitindo que a chapa esteja submetida a pequenas deformações, podem-se inferir as seguintes relações geométricas.

$B^{\prime} C^{\prime}=\left(1+\varepsilon_{s x)} \cdot \operatorname{sen} \theta\right.$

$A^{\prime} C^{\prime}=\left(1+\varepsilon_{s y)} \cdot \cos \theta\right.$

$B^{`} D^{`}=\left(1-\varepsilon_{c}\right) \cdot \operatorname{sen}^{2} \theta$

$A^{`} D^{`}=\left(1-\varepsilon_{c}\right) \cdot \cos ^{2} \theta$

$A^{`} B^{`}=\left(1-\varepsilon_{c}\right)$

$E$ :

$D C^{\prime 2}=B^{\prime} C^{\prime 2}-B^{\prime} D^{2}=A^{\prime} C^{\prime 2}-A^{\prime} D^{2}$

Substituindo:

$\left[\left(1+\varepsilon_{s x}\right) \cdot \operatorname{sen} \theta\right]^{2}-\left[\left(1-\varepsilon_{c}\right) \cdot \operatorname{sen}^{2} \theta\right]^{2}=\left[\left(1+\varepsilon_{s y}\right) \cdot \cos \theta\right]^{2}-\left[\left(1-\varepsilon_{c}\right) \cdot \cos ^{2} \theta\right]^{2}$ 
$\left(1+2 \cdot \varepsilon_{s x}+\varepsilon_{s x}^{2}\right) \cdot \operatorname{sen}^{2} \theta-\left(1-2 \cdot \varepsilon_{c}+\varepsilon_{c}^{2}\right) \cdot \operatorname{sen}^{4} \theta$

$$
=\left(1+2 \cdot \varepsilon_{s y}+\varepsilon_{s y}^{2}\right) \cdot \cos ^{2} \theta-\left(1-2 \cdot \varepsilon_{c}+\varepsilon_{c}^{2}\right) \cdot \cos ^{4} \theta
$$

Como neste caso, as deformações são pequenas, pode-se utilizar a equação de forma aproximada, eliminando os termos de ordem superior. Assim, tem-se que:

$\left(1+2 \cdot \varepsilon_{s x}\right) \cdot \operatorname{sen}^{2} \theta-\left(1-2 \cdot \varepsilon_{c}\right) \cdot \operatorname{sen}^{4} \theta=\left(1+2 \cdot \varepsilon_{s y}\right) \cdot \cos ^{2} \theta-\left(1-2 \cdot \varepsilon_{c}\right) \cdot \cos ^{4} \theta$

Dividindo por $\cos ^{2} \theta$ :

$$
\begin{aligned}
& \left(1+2 \cdot \varepsilon_{s x}\right) \cdot \operatorname{tg}^{2} \theta-\left(1-2 \cdot \varepsilon_{c}\right) \cdot \operatorname{tg}^{2} \theta \cdot \operatorname{sen}^{2} \theta=\left(1+2 \cdot \varepsilon_{s y}\right)-\left(1-2 \cdot \varepsilon_{c}\right) \cdot \cos ^{2} \theta \\
& \operatorname{tg} \theta+2 \varepsilon_{s x} \cdot \operatorname{tg}^{2} \theta-\operatorname{sen}^{2} \theta \cdot \operatorname{tg}^{2} \theta+2 \varepsilon_{c} \cdot \operatorname{sen}^{2} \theta \cdot \operatorname{tg}^{2} \theta+\cos ^{2} \theta-2 \cdot \varepsilon_{c} \cdot \cos ^{2} \theta=1+2 \cdot \varepsilon_{s y} \\
& 2 \varepsilon_{s y}-2 \varepsilon_{s x} \cdot \operatorname{tg}^{2} \theta+\left(1-\cos ^{2} \theta-\operatorname{tg}^{2} \theta+\operatorname{sen}^{2} \theta \cdot \operatorname{tg}^{2} \theta\right)+2 \varepsilon_{c} \cdot\left(\cos ^{2} \theta-\operatorname{sen}^{2} \theta \cdot \operatorname{tg}^{2} \theta\right)=0 \\
& 2 \varepsilon_{s y}-2 \cdot \varepsilon_{s x} \cdot \operatorname{tg}^{2} \theta+\left(\operatorname{sen}^{2} \theta-\operatorname{tg}^{2} \theta \cdot\left(1-\operatorname{sen}^{2} \theta\right)\right)+2 \cdot \varepsilon_{c} \cdot\left(1-\operatorname{sen}^{2} \theta-\operatorname{sen}^{2} \theta \cdot \operatorname{tg}^{2} \theta\right) \\
& \quad=0 \\
& 2 \varepsilon_{s y}-2 \cdot \varepsilon_{s x} \cdot \operatorname{tg}^{2} \theta+\left(\operatorname{sen}^{2} \theta-\operatorname{tg}^{2} \theta \cdot \cos ^{2} \theta\right)+2 \cdot \varepsilon_{c} \cdot\left(1-\operatorname{sen}^{2} \theta\left(1+\operatorname{tg}^{2} \theta\right)\right)=0 \\
& 2 \varepsilon_{s y}-2 \cdot \varepsilon_{s x} \cdot \operatorname{tg}^{2} \theta+\left(\operatorname{sen}^{2} \theta-\operatorname{sen}^{2} \theta\right)+2 \cdot \varepsilon_{c} \cdot\left(1-\frac{\operatorname{tg}^{2} \theta}{1+\operatorname{tg}^{2} \theta} \cdot\left(1+\operatorname{tg}^{2} \theta\right)\right)=0 \\
& 2 \varepsilon_{s y}-2 \cdot \varepsilon_{s x} \cdot \operatorname{tg}^{2} \theta+2 \cdot \varepsilon_{c} \cdot\left(1-\operatorname{tg}^{2} \theta\right)=0 \\
& \varepsilon_{s y}-\varepsilon_{s x} \cdot \operatorname{tg}^{2} \theta-\varepsilon_{c} \cdot \operatorname{tg}^{2} \theta \cdot\left(1-\operatorname{cotg}^{2} \theta\right)=0 \\
& \varepsilon_{s y}=\varepsilon_{s x} \cdot \operatorname{tg}^{2} \theta+\varepsilon_{c} \cdot \operatorname{tg}^{2} \theta \cdot\left(1-\operatorname{cotg}^{2} \theta\right) \\
& \varepsilon_{s y}=\varepsilon_{s x} \cdot \operatorname{tg}^{2} \theta \cdot\left[1+\frac{\varepsilon_{c}}{\varepsilon_{s x}} \cdot\left(1-\operatorname{cotg}^{2} \theta\right)\right] \\
& \frac{\varepsilon_{s y}}{\varepsilon_{s x}}=\operatorname{tg}^{2} \theta \cdot\left[1+\frac{\varepsilon_{c}}{\varepsilon_{s x}} \cdot\left(1-\operatorname{cotg}^{2} \theta\right)\right]
\end{aligned}
$$

\subsubsection{Dimensionamento econômico}

De forma a se obter a maior eficiência das armaduras, para o caso em que há armaduras em ambas as direções, elas devem escoar ao mesmo tempo no Estado 
Limite Último. Assim, $\varepsilon_{\text {sy }}$ e $\varepsilon_{s x}$ devem ser iguais à deformação de escoamento. Portanto, a eq. 2.5 fica:

$1=\operatorname{tg}^{2} \theta \cdot\left[1+\frac{\varepsilon_{c}}{\varepsilon_{s x}} \cdot\left(1-\operatorname{cotg}^{2} \theta\right)\right]$

Assim, da equação 2.6, tem-se que $\theta$ deve ser igual $45^{\circ}$ para obter 0 dimensionamento mais eficiente e, portanto, econômico.

Além disso, é possível demonstrar que, ao se utilizar $\theta$ com o valor de $45^{\circ}$, a tensão de compressão no concreto é a menor possível.

Retomando a equação 2.4, derivando-a em função de $\theta$ e igualando-a a zero de forma a encontrar o extremo dessa função, tem-se que.

$$
\begin{aligned}
& n_{c}=n_{x y}(\operatorname{tg} \theta+\operatorname{cotg} \theta) \\
& \frac{\partial n_{c}}{\partial \theta}=n_{x y} \cdot\left(\frac{1}{\cos ^{2} \theta}-\frac{1}{\operatorname{sen}^{2} \theta}\right)=0 \\
& \frac{\operatorname{sen}^{2} \theta-\cos ^{2} \theta}{\operatorname{sen}^{2} \theta \cdot \cos ^{2} \theta}=0 \\
& \operatorname{sen} \theta=\cos \theta \Rightarrow \theta=45^{\circ}
\end{aligned}
$$

Portanto, quando $\theta$ é igual a $45^{\circ}$, a função está em um extremo, o qual é possível demonstrar que este é um extremo mínimo se $\mathrm{n}_{\mathrm{xy}}$ for positivo, em que $\mathrm{n}_{\mathrm{c}}$ vale:

$$
\begin{aligned}
& n_{c}=n_{x y}\left(\operatorname{tg} 45^{\circ}+\operatorname{cotg} 45^{\circ}\right) \\
& n_{c}=2 . n_{x y}
\end{aligned}
$$

Para os casos em que $n_{x y}$ é negativo, o valor de $n_{c}$ seria negativo para $\theta=45^{\circ}$ o que fisicamente significaria que o concreto está submetido a esforços de tração. Isto não é verdade. O que de fato ocorre é que, conforme mostrado nas Figura 2.13 e Figura 2.14, o ângulo $\theta$ passa a ser negativo e o concreto é comprimido na direção perpendicular e, assim, $\mathrm{n}_{\mathrm{c}}$ continuam assumindo valores apenas positivos. 

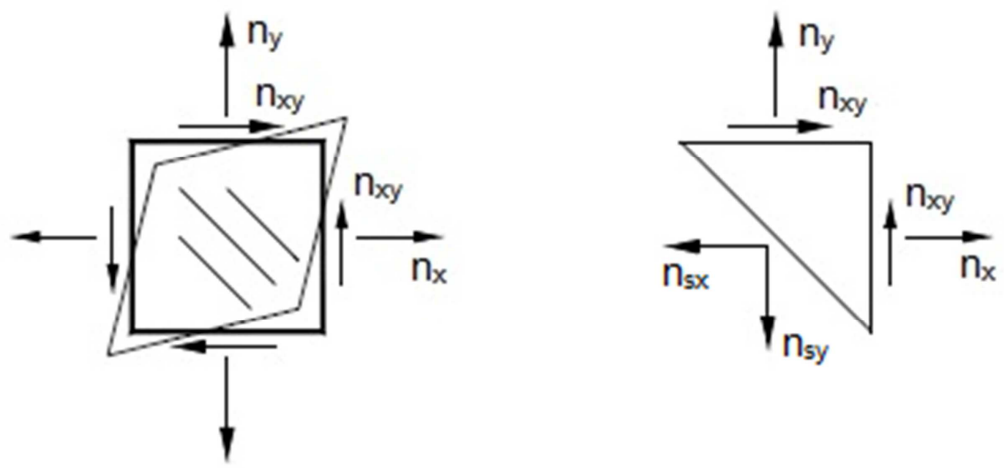

Figura 2.13 - Configuração deformada e fissuras em chapa submetida a esforço de cisalhamento positivo (adaptado de CHEN, 2004)
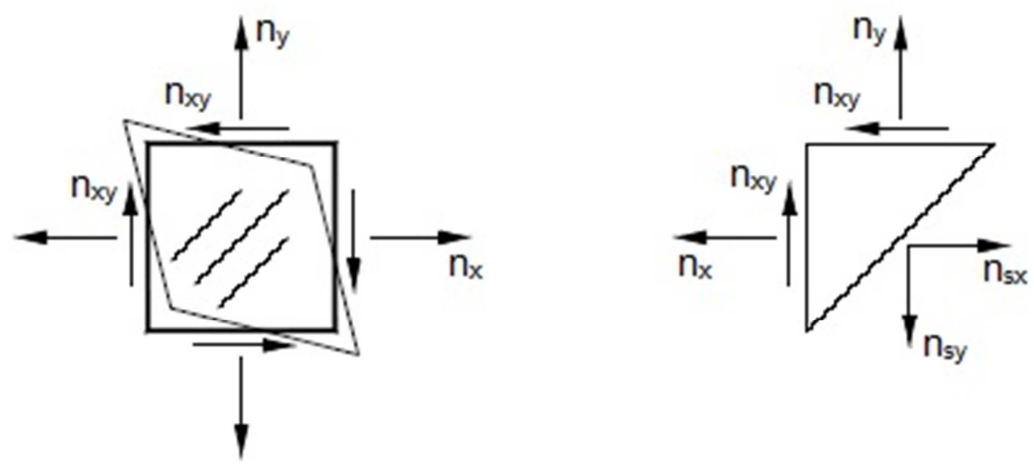

Figura 2.14 - Configuração deformada e fissuras em chapa submetida a esforço de cisalhamento negativo (adaptado de CHEN, 2004)

Portanto, para englobar os dois casos, a equação 2.7 deve ser:

$n_{c}=2 \cdot\left|n_{x y}\right|$

De forma ilustrativa, sendo $\mathrm{n}_{\mathrm{xy}}$ igual a $100 \mathrm{kN}$, o gráfico obtido para a equação 2.8 é $o$ apresentado na Figura 2.15. Pode-se notar que em $45^{\circ}$ a função assume seu valor mínimo, sendo, para este caso igual a $200 \mathrm{kN}$. 


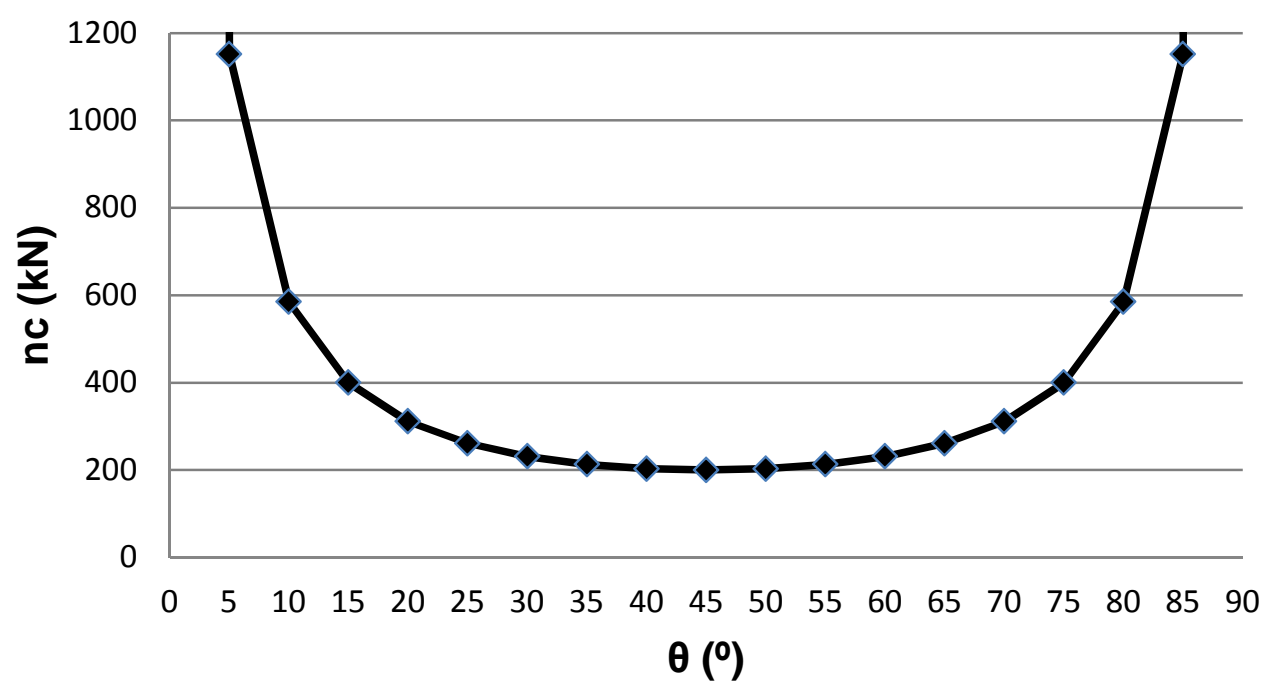

Figura 2.15 - Gráfico da equação 2.5 para $\mathrm{n}_{\mathrm{xy}}$ de $100 \mathrm{kN}$

Com as equações apresentadas e admitindo dimensionamento econômico, adotando, assim, $\theta=45^{\circ}$, aplicando nas eq. 2.1 e 2.2, tem-se que:

$$
\begin{aligned}
& n_{s x}=n_{x}+n_{x y} \cdot \operatorname{tg} \theta \\
& n_{s x}=n_{x}+n_{x y} \\
& n_{s y}=n_{y}+n_{x y} \cdot \operatorname{cotg} \theta \\
& n_{s y}=n_{y}+n_{x y}
\end{aligned}
$$

Conforme apresentado, se o sentido de $\mathrm{n}_{\mathrm{xy}}$ for invertido, ou seja, assumir valores negativos, $\theta$ deverá ser igual a $-45^{\circ}$. Assim, as expressões que contemplam esses casos são:

$$
\begin{aligned}
& n_{s x}=n_{x}+\left|n_{x y}\right| \\
& n_{s y}=n_{y}+\left|n_{x y}\right|
\end{aligned}
$$




\subsubsection{Resistência de projeto dos materiais}

\subsubsection{Aço}

Para o aço, é considerado, tanto para este método apresentado, quanto para o restante do trabalho, o diagrama tensão-deformação bilinear que consta na NBR 6118 apresentado na Figura 2.16, onde $\mathrm{f}_{\mathrm{yk}}$ é a resistência última característica do aço, $f_{y d}$ sua resistência de cálculo e $E_{c s}$ é o módulo de elasticidade que vale 210 GPa.

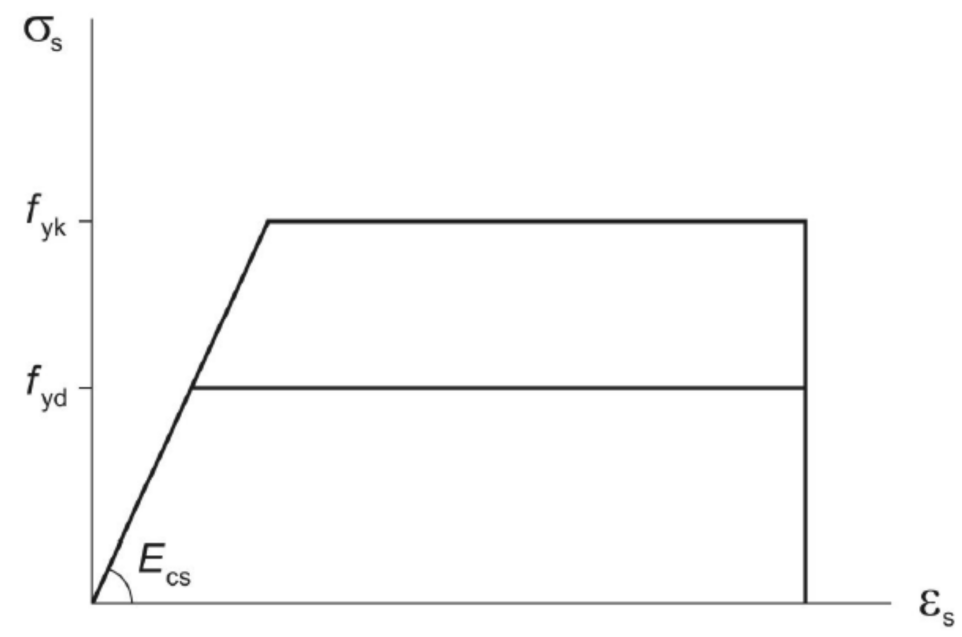

Figura 2.16 - Diagrama tensão-deformação para o aço (NBR-6118:2003)

A NBR6118 determina que os aços de armadura passiva devam ser de categoria CA-25, CA-50 e CA-60. A Tabela 2.1 mostra a resistência e a deformação de escoamento para estes aços.

Tabela 2.1 - Resistências dos aços e deformação de escoamento (NBR 6118)

\begin{tabular}{|c|c|c|c|}
\hline & $\mathrm{f}_{\mathrm{yk}}\left(\mathrm{kN} / \mathrm{cm}^{2}\right)$ & $\mathrm{f}_{\mathrm{yd}}\left(\mathrm{kN} / \mathrm{cm}^{2}\right)$ & $\varepsilon_{\mathrm{yd}}(\% \circ)$ \\
\hline CA-25 & 25 & 21,74 & 1,04 \\
\hline CA-50 & 50 & 43,48 & 2,07 \\
\hline CA-60 & 60 & 52,17 & 2,48 \\
\hline
\end{tabular}




\subsubsection{Concreto}

O CEB (1990) recomenda o uso de valores fixos para a resistência do concreto para o dimensionamento de chapas. Para os casos em que o concreto não está fissurado, sugere-se adotar $o$ valor de $f_{c d 1}$ e nos casos em que há fissuração $o$ valor de $f_{c d 2}$, sendo eles:

$$
\begin{aligned}
& f_{c d 1}=0,85 \cdot\left(1-\frac{f_{c k}}{250}\right) \cdot f_{c d} \\
& f_{c d 2}=0,60 \cdot\left(1-\frac{f_{c k}}{250}\right) \cdot f_{c d}
\end{aligned}
$$

Estes valores são normalmente utilizados quando se dimensiona chapas através do método baseado nos critério de Baumann, por isso ele será apresentado considerando-os.

Porém, para o estudo das armaduras de compressão, no item 3.1 será descrita uma forma de admitir a resistência do concreto de forma que leve em conta não apenas se o concreto está fissurado ou não, mas sim a deformação de tração que ocorre na direção perpendicular à compressão no concreto para que, desta forma, se alcance resistências intermediárias entre esses dois valores.

\subsubsection{Casos de dimensionamento}

O CEB (1990) separa o dimensionamento das chapas em quatro casos. O primeiro deles, caso I, considera as armaduras nas duas direções tracionadas, portanto tanto $\mathrm{n}_{\mathrm{sx}}$ quanto $\mathrm{n}_{\text {sy }}$ serão positivos.

Quando um dos esforços nas armaduras assumir valores negativos, ou seja, de compressão, pode-se dispensar o uso de armação. Se não houver esforço de tração na direção x, se aplica o caso II de dimensionamento. Se não houver na direção y, se aplica o caso III. 
Quando a chapa está completamente comprimida, se recai no caso IV, para o qual não é necessária armadura alguma.

Para todos os casos, este método de dimensionamento não contempla a utilização de armaduras de compressão. A Figura 2.17 mostra o resumo dos casos de dimensionamento.
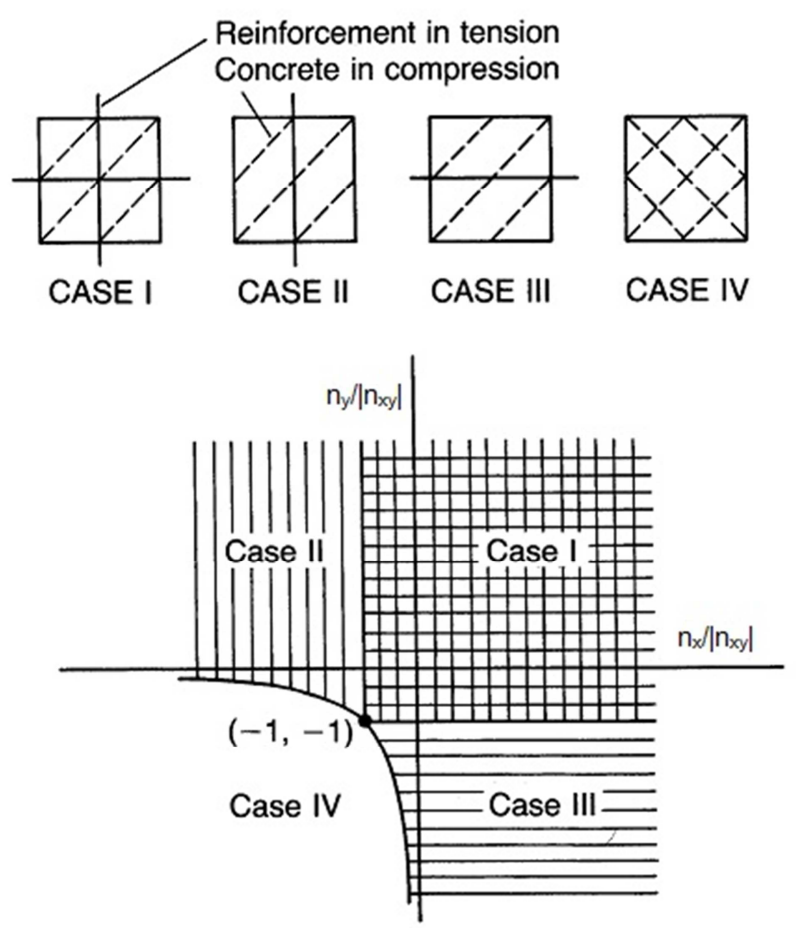

Figura 2.17 - Resumo dos casos de dimensionamento de chapas (adaptado CEB, 1990)

\subsubsection{Caso I - Armaduras nas duas direções}

Retomando as equações 2.11 e 2.12, para que sejam necessárias as armaduras nas duas direções, as seguintes condições devem ser satisfeitas.

$$
\begin{aligned}
& n_{s x}=n_{x}+\left|n_{x y}\right|>0 \\
& n_{s y}=n_{y}+\left|n_{x y}\right|>0
\end{aligned}
$$

Assim, o dimensionamento das armaduras é definido por: 
$a_{s x}=\frac{n_{s x}}{f_{y d}}=\frac{n_{x}+\left|n_{x y}\right|}{f_{y d}}$

$a_{s y}=\frac{n_{s y}}{f_{y d}}=\frac{n_{y}+\left|n_{x y}\right|}{f_{y d}}$

Para a verificação do concreto no caso I será utilizado o valor de $\mathrm{f}_{\mathrm{cd} 2}$ para a resistência à compressão porque, neste caso, o concreto está fissurado. Assim, a expressão é a seguinte.

$$
\begin{aligned}
& \sigma_{c}=\frac{n_{c}}{h} \leq f_{c d 2} \\
& \frac{2\left|n_{x y}\right|}{h} \leq 0,6\left(1-\frac{f_{c k}}{250}\right) \cdot f_{c d}
\end{aligned}
$$

\subsubsection{Caso II - Armadura apenas na direção y}

Para que se possa utilizar armadura apenas da direção y, o esforço resistente $n_{s x}$ deve ser negativo, enquanto $\mathrm{n}_{\text {sy }}$ positivo, satisfazendo as expressões seguintes.

$$
\begin{aligned}
& n_{s x}=n_{x}+\left|n_{x y}\right| \leq 0 \\
& n_{s y}=n_{y}+\left|n_{x y}\right|>0
\end{aligned}
$$

Assim, dispensando a armadura na direção $\mathrm{x}, \mathrm{n}_{\mathrm{sx}}$ será nulo, portanto, voltando nas equações 2.1 e 2.2, tem-se que:

$$
\begin{aligned}
& n_{s x}=n_{x}+n_{x y} \cdot \operatorname{tg} \theta=0 \\
& n_{s y}=n_{y}+n_{x y} \cdot \operatorname{cotg} \theta
\end{aligned}
$$

O ângulo $\theta$ não será mais $45^{\circ}$, mas será determinado pela equação 2.1 , ficando assim: 
$\operatorname{tg} \theta=-\frac{n_{x}}{n_{x y}}$

$\theta=\operatorname{arctg}\left(-\frac{n_{x}}{n_{x y}}\right)$

Continuando, substituindo a equação 2.19 na equação 2.18 tem-se que:

$n_{s y}=n_{y}-\frac{n_{x y}{ }^{2}}{n_{x}}$

Para o esforço de compressão no concreto, tem-se que:

$n_{c}=n_{x y} \cdot(\operatorname{tg} \theta+\operatorname{cotg} \theta)$

$n_{c}=n_{x y} \cdot\left(-\frac{n_{x}}{n_{x y}}-\frac{n_{x y}}{n_{x}}\right)$

$n_{c}=-n_{x}-\frac{n_{x y}{ }^{2}}{n_{x}}$

Resumindo, o dimensionamento das armaduras é determinado pelas expressões:

$$
a_{s x}=0
$$

$a_{s y}=\frac{n_{s y}}{f_{y d}}=\frac{n_{y}-\frac{n_{x y}{ }^{2}}{n_{x}}}{f_{y d}}$

$\mathrm{Na}$ verificação do concreto, se utilizará o $\mathrm{f}_{\mathrm{cd} 2}$ pelo mesmo motivo apresentado para o caso I, ficando assim:

$$
\begin{aligned}
& \sigma_{c}=\frac{n_{c}}{h} \leq f_{c d 2} \\
& \frac{-n_{x}-\frac{n_{x y}{ }^{2}}{n_{x}}}{h} \leq 0,6\left(1-\frac{f_{c k}}{250}\right) \cdot f_{c d}
\end{aligned}
$$




\subsubsection{Caso III - Armadura apenas na direção x}

O caso III é semelhante ao caso II, diferenciando apenas a direção da armadura. Então, para se ter armadura apenas na direção $x$, as seguintes inequações devem ser satisfeitas.

$$
\begin{aligned}
& n_{s x}=n_{x}+\left|n_{x y}\right|>0 \\
& n_{s y}=n_{y}+\left|n_{x y}\right| \leq 0
\end{aligned}
$$

Da mesma forma, dispensando a armadura na direção $\mathrm{y}, \mathrm{n}_{\mathrm{sy}}$ será nulo, portanto, voltando nas eq. 2.1 e 2.2 , tem-se que:

$$
\begin{aligned}
& n_{s x}=n_{x}+n_{x y} \cdot \operatorname{tg} \theta \\
& n_{s y}=n_{y}+n_{x y} \cdot \operatorname{cotg} \theta=0
\end{aligned}
$$

O ângulo $\theta$ não será mais $45^{\circ}$, mas será determinado pela equação 2.26 , ficando assim:

$$
\begin{aligned}
& \operatorname{cotg} \theta=-\frac{n_{y}}{n_{x y}} \\
& \theta=\operatorname{arctg}\left(-\frac{n_{x y}}{n_{y}}\right)
\end{aligned}
$$

Substituindo a eq. 2.27 na eq. 2.25 tem-se que:

$$
n_{s x}=n_{x}-\frac{n_{x y}{ }^{2}}{n_{y}}
$$

Para o esforço de compressão no concreto, tem-se que:

$$
\begin{aligned}
& n_{c}=n_{x y \cdot}(\operatorname{tg} \theta+\operatorname{cotg} \theta) \\
& n_{c}=n_{x y} \cdot\left(-\frac{n_{x y}}{n_{y}}-\frac{n_{y}}{n_{x y}}\right)
\end{aligned}
$$


$n_{c}=-n_{y}-\frac{n_{x y}{ }^{2}}{n_{y}}$

O dimensionamento das armaduras fica assim:

$a_{s x}=\frac{n_{s x}}{f_{y d}}=\frac{n_{x}-\frac{n_{x y}{ }^{2}}{n_{y}}}{f_{y d}}$

$a_{s y}=0$

A verificação do concreto é dada pela expressão:

$$
\begin{aligned}
& \sigma_{c}=\frac{n_{c}}{h} \leq f_{c d 2} \\
& \frac{-n_{y}-\frac{n_{x y}{ }^{2}}{n_{y}}}{h} \leq 0,6\left(1-\frac{f_{c k}}{250}\right) \cdot f_{c d}
\end{aligned}
$$

\subsubsection{Caso IV - Dispensa de armadura nas duas direções}

Para que se possam dispensar as armaduras, não pode haver tensões de tração na peça, portanto as condições de carregamento devem respeitar as seguintes inequações.

$$
\begin{aligned}
& n_{s x}=n_{x}+\left|n_{x y}\right| \leq 0 \\
& n_{s y}=n_{y}+\left|n_{x y}\right| \leq 0
\end{aligned}
$$

Desta forma, tem-se que:

$$
\begin{aligned}
& n_{x} \leq-\left|n_{x y}\right| \\
& n_{y} \leq-\left|n_{x y}\right|
\end{aligned}
$$

Multiplicando as inequações 2.33 com 2.34, tem-se que: 
$n_{x} \cdot n_{y} \geq n_{x y}^{2}$

Assim, outra forma de expressar as condições 2.31 e 2.32 está mostrada pelas equações $2.35,2.36$ e 2.37 .

$$
\begin{aligned}
& n_{x} \leq 0 \\
& n_{y} \leq 0 \\
& n_{x} \cdot n_{y} \geq n_{x y}{ }^{2}
\end{aligned}
$$

Neste caso, deve-se apenas verificar se o concreto irá resistir à compressão na direção principal. Para tanto, irar-se-á analisar o equilíbrio mostrado na Figura 2.18.

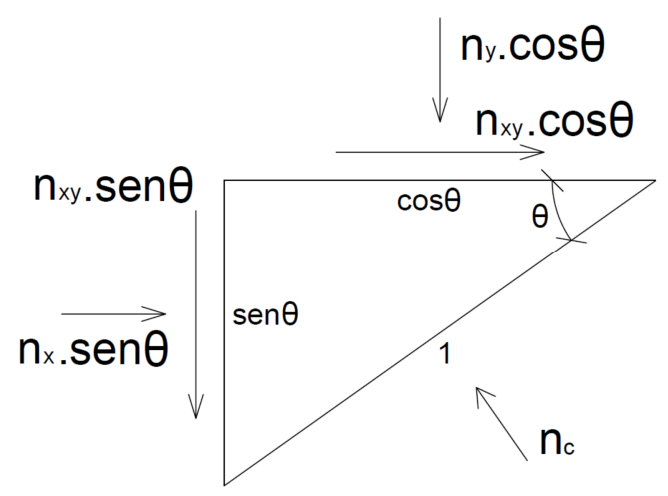

Figura 2.18 - Equilíbrio de forças na seção perpendicular a força de compressão do concreto

Fazendo a somatório das forças em x, tem-se que:

$$
\begin{aligned}
& \sum F_{x}=0 \\
& -n_{x \cdot} \operatorname{sen} \theta+n_{x y} \cdot \cos \theta-n_{c} \cdot \operatorname{sen} \theta=0 \\
& -n_{x}+n_{x y} \cdot \frac{\cos \theta}{\operatorname{sen} \theta}-n_{c}=0 \\
& -n_{x}+n_{x y} \cdot \operatorname{cotg} \theta-n_{c}=0 \\
& \operatorname{cotg} \theta=\frac{n_{c}+n_{x}}{n_{x y}}
\end{aligned}
$$

Agora, somando em y, tem-se que: 
$\sum F_{y}=0$

$-n_{y} \cdot \cos \theta+n_{x y} \cdot \operatorname{sen} \theta-n_{c} \cdot \cos \theta=0$

$n_{c}=-n_{y}+n_{x y} \cdot \operatorname{tg} \theta$

Aplicando a eq. 2.28 em eq. 2.29

$$
\begin{aligned}
& n_{c}=-n_{y}+n_{x y} \cdot\left(\frac{n_{x y}}{n_{c}+n_{x}}\right) \\
& n_{c}+n_{y}=\left(\frac{n_{x y}{ }^{2}}{n_{c}+n_{x}}\right) \\
& \left(n_{c}+n_{y}\right) \cdot\left(n_{c}+n_{x}\right)=n_{x y}{ }^{2} \\
& n_{c}{ }^{2}+\left(n_{x}+n_{y}\right) \cdot n_{c}+n_{x} \cdot n_{y}-n_{x y}{ }^{2}=0
\end{aligned}
$$

Resolvendo a equação de $2^{\circ}$ grau, tem-se que:

$$
\begin{aligned}
& \Delta=\left(n_{x}+n_{y}\right)^{2}-4 \cdot\left(n_{x} \cdot n_{y}-n_{x y}{ }^{2}\right) \\
& \Delta=n_{x}{ }^{2}+2 \cdot n_{x} \cdot n_{y}+n_{y}{ }^{2}-4 \cdot n_{x} \cdot n_{y}+4 \cdot n_{x y}{ }^{2}=0 \\
& \Delta=n_{x}{ }^{2}-2 \cdot n_{x} \cdot n_{y}+n_{y}{ }^{2}+4 \cdot n_{x y}{ }^{2}=0 \\
& \Delta=\left(n_{x}-n_{y}\right)^{2}+4 \cdot n_{x y}{ }^{2}=0
\end{aligned}
$$

Portanto:

$n_{c}=\frac{-n_{x}+n_{y} \pm \sqrt{\left(n_{x}-n_{y}\right)^{2}+4 . n_{x y}{ }^{2}}}{2}$

$n_{c}=-\frac{n_{x}+n_{y}}{2} \pm \sqrt{\frac{\left(n_{x}-n_{y}\right)^{2}}{4}+n_{x y}{ }^{2}}$

Para esta verificação, apenas importa a solução com sinal positivo da equação 2.40, pois esta resulta no maior esforço de compressão para o qual a chapa está submetida. A solução com sinal negativo é justamente o oposto, ou seja, corresponde ao valor de menor esforço de compressão. Nesta verificação, 
diferentemente dos outros casos, será utilizado como valor de referência para resistência do concreto o $f_{c d 1}$ do CEB (1990), pois neste caso não há fissuração. Desta forma, tem-se que:

$$
\begin{aligned}
& \sigma_{c}=\frac{n_{c}}{h} \leq f_{c d 1} \\
& \left(-\frac{n_{x}+n_{y}}{2}+\sqrt{\frac{\left(n_{x}-n_{y}\right)^{2}}{4}+n_{x y}^{2}}\right) \cdot \frac{1}{h} \leq f_{c d 1} \\
& \left(-\frac{n_{x}+n_{y}}{2}+\sqrt{\frac{\left(n_{x}-n_{y}\right)^{2}}{4}+n_{x y}^{2}}\right) \cdot \frac{1}{h} \leq 0,85 \cdot\left(1-\frac{f_{c k}}{250}\right) \cdot f_{c d}
\end{aligned}
$$

\subsection{Considerações sobre o estudo das armaduras de compressão}

Tendo em mente os casos de dimensionamento propostos pelo CEB (1990) apresentados, pode-se analisar em quais deles é coerente estudar armaduras de compressão.

Primeiramente, para o caso I, armaduras de compressão só poderiam ser utilizadas com eficácia se fossem dispostas na direção das fissuras, onde ajudariam a reduzir a tensão de compressão no concreto. Entretanto, como neste caso já há uma malha ortogonal de armaduras, a colocação de mais uma camada de barras em outra direção tornaria a solução construtivamente ruim, somente aconselhável em casos de peças especiais.

Outra possibilidade seria superdimensionar a chapa, ou seja, colocar maior quantidade de armadura de forma a limitar a deformação de compressão e, consequentemente, diminuir a tensão na direção principal. Entretanto, essa solução levaria a uma ruptura frágil da peça, já que o concreto romperia antes das armaduras escoarem. 
Para os casos II e III, é razoável pensar que ao se colocar armadura na direção que a priori não foi armada, ela afetará a distribuição de tensões da chapa de forma a diminuir a tensão de compressão no concreto.

Já para o caso IV, onde não há armadura alguma, é evidente que, ao se dispor barras de forma adequada nessa chapa, ela ajudará a combater os esforços de compressão.

Portanto, neste estudo somente serão abordados os casos II, III e IV de dimensionamento. 


\section{MODELO RESISTENTE E VERIFICAÇÃO DA TENSÃO DE COMPRESSÃO NO CONCRETO}

\subsection{Modelo resistente para o concreto em compressão}

No método baseado nos critério de Baumann apresentado no Capítulo 2, os valores de resistência do concreto seguiram aqueles recomendados pelo CEB (1990), apresentados no item 2.3.4.2.

Resumidamente, o CEB (1990) recomenda duas formas de considerar a tensão e a resistência do concreto para a situação em que ele se encontra uniaxialmente comprimido. A primeira forma é utilizar o diagrama parábola-retângulo, apresentado na Figura 3.1. Esta possibilidade só pode ser aplicada para casos em que o concreto está sem fissuras.

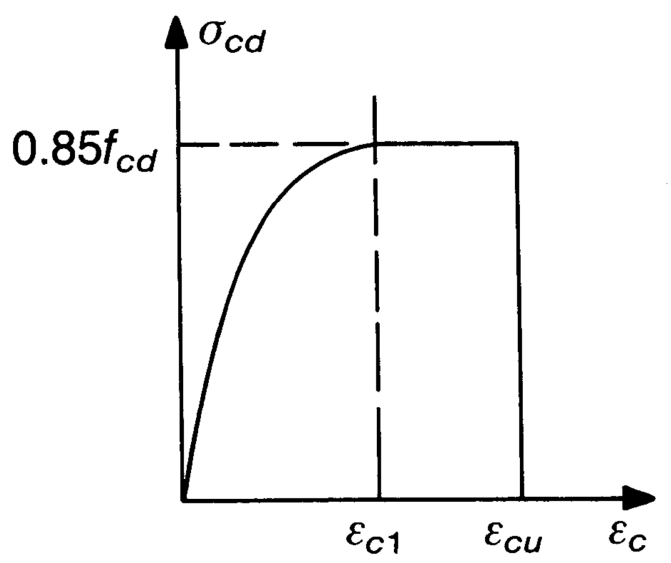

Figura 3.1 - Diagrama tensão-deformação parábola-retângulo para o concreto comprimido uniaxialmente (CEB, 1990)

Este diagrama é representado pelas seguintes equações:

$$
\begin{array}{ll}
\sigma_{c d}=0.85 . f_{c d} \cdot\left[2 \cdot\left(\frac{\varepsilon_{c}}{\varepsilon_{c 1}}\right)-\left(\frac{\varepsilon_{c}}{\varepsilon_{c 1}}\right)^{2}\right] & \text { para }\left|\varepsilon_{c}\right|<\left|\varepsilon_{c 1}\right| \\
\sigma_{c d}=0.85 . f_{c d} & \text { para }\left|\varepsilon_{c 1}\right| \leq\left|\varepsilon_{c}\right| \leq\left|\varepsilon_{c u}\right|
\end{array}
$$


$\sigma_{c d}=0$

para $\left|\varepsilon_{\mathrm{cu}}\right|<\left|\varepsilon_{\mathrm{c}}\right|$

Onde $\varepsilon_{c 1}=-2 \%$

Para casos em que a peça está sujeita a flexão é recomendado considerar:

$\varepsilon_{c u}=-3,5 \%$ para $\mathrm{f}_{c k} \leq 50 \mathrm{MPa}$

$\varepsilon_{c u}=-3,5 \%$ o. $\left(\frac{50}{f_{c k}}\right) \quad$ para $50 \mathrm{MPa}<\mathrm{f}_{\mathrm{ck}} \leq 80 \mathrm{MPa}$

Para caso em que o concreto está submetido à compressão axial:

$\varepsilon_{c u}=-2 \%$

Este equacionamento é o mesmo utilizado pela NBR 6118. A outra forma recomendada pelo CEB (1990) de considerar a resistência do concreto é utilizar um diagrama de tensão uniforme. Esta possibilidade pode ser aplicada para o caso do concreto estar intacto, assumindo assim o valor de $f_{c d 1}$, ou para quando o concreto está fissurado, assumindo o valor de $\mathrm{f}_{\mathrm{cd} 2}$. Desta forma, o CEB (1990) contempla o fenômeno do amolecimento do concreto fissurado, que consiste justamente na perda de resistência à compressão devido à fissuração.

$$
\begin{aligned}
& f_{c d 1}=0,85 \cdot\left(1-\frac{f_{c k}}{250}\right) \cdot f_{c d} \\
& f_{c d 2}=0,60 \cdot\left(1-\frac{f_{c k}}{250}\right) \cdot f_{c d}
\end{aligned}
$$

Estes valores são válidos para quando a deformação de qualquer fibra da área a ser aplicada esta tensão não ultrapasse $\varepsilon^{*}{ }_{\text {cu. }}$.

$$
\varepsilon_{c u}^{*}=-\left(4 \% 0-2 \% 0 \cdot\left(\frac{f_{c k}}{100}\right)\right)
$$


Para o caso especifico das chapas, o CEB (1990) recomenda o uso do diagrama uniforme para definição da resistência de projeto. Entretanto, o uso destes valores no dimensionamento das chapas impõe uma descontinuidade entre os casos em que o concreto está fissurado e aquele em que o concreto está intacto.

Isto posto, neste trabalho será adotado um modelo resistente para o concreto que otimize o uso do material. Para tanto, se deseja interpolar o valor máximo da resistência entre $f_{c d 1}$ e $f_{c d 2}$.

Vecchio e Collins (1986) propõe uma formulação que contempla o amolecimento do concreto devido à sua fissuração de forma diferente da do CEB (1990). Para eles a perda de resistência do concreto fissurado está relacionada com a deformação de tração imposta na região em questão. A Figura 3.2 expressa essa relação graficamente.

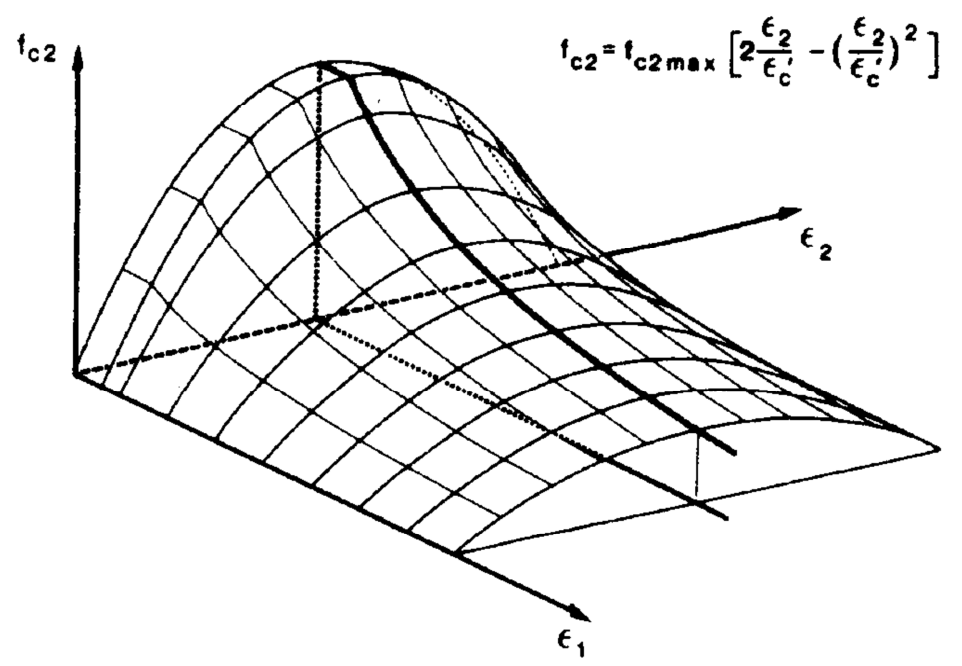

Figura 3.2 - Representação tridimensional do diagrama tensão-deformação do concreto em compressão (VECCHIO E COLLINS, 1986)

A partir da Figura 3.2, pode se ver que quanto maior a deformação de tração $\varepsilon_{1}$, menor será a resistência do concreto a compressão na direção 2, aquela submetida a compressão. A equação 3.2 é a expressão proposta por Vecchio e Collins (1986) que determina essa perda. $O$ valor $f_{c 2 m a x}$ representa resistência à compressão do concreto. Sua representação gráfica é mostrada na Figura 3.3. 


$$
f_{c 2 \max }=\frac{f_{c}^{\prime}}{0,8-0,34 \cdot \frac{\varepsilon_{1}}{\varepsilon_{c}}} \leq f_{c}^{\prime}
$$

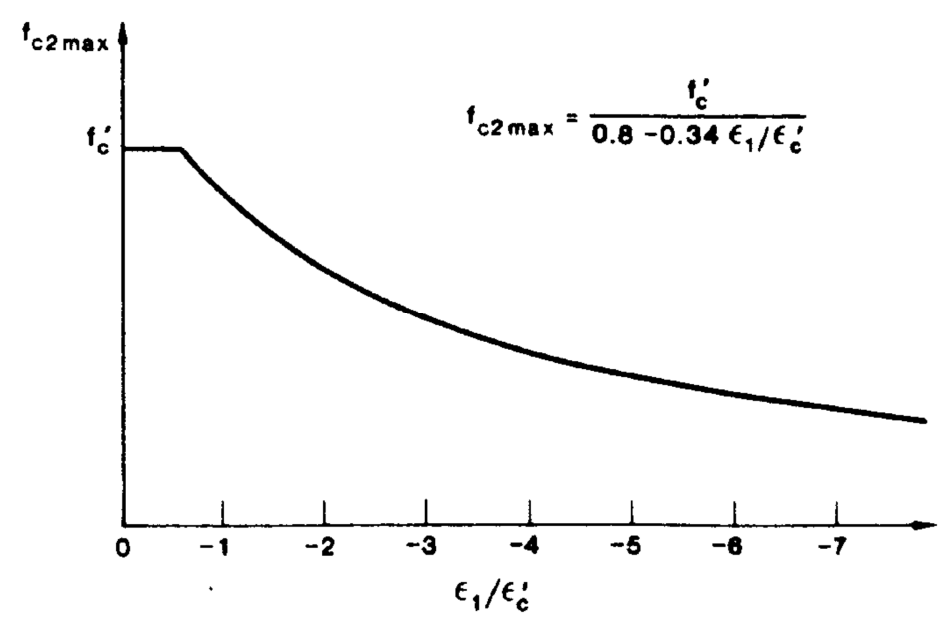

Figura 3.3 - Redução da tensão de pico à compressão do concreto (VECCHIO E COLLINS, 1986)

O CEB (1990) e Vecchio e Collins (1986) utilizam como valor de deformação correspondente a tensão máxima de compressão no concreto:

$\varepsilon_{c 1}=\varepsilon_{c}^{\prime}=-2 \%$

Assim, substituindo esse valor na eq. 3.2, tem-se que:

$f_{c 2 \max }=\frac{f_{c}^{\prime}}{0,8+170 . \varepsilon_{1}} \leq f_{c}^{\prime}$

Já o equacionamento da parábola da curva do diagrama tensão-deformação do concreto é semelhante à proposta pelo CEB (1990), tendo como diferença o pico da função. Esta função é a apresentada na eq. 3.4 e seu gráfico é representado na Figura 3.4.

$$
f_{c 2}=\sigma_{c}=f_{c 2 \max } \cdot\left[2 \cdot\left(\frac{\varepsilon_{2}}{\varepsilon_{c}^{\prime}}\right)-\left(\frac{\varepsilon_{2}}{\varepsilon_{c}^{\prime}}\right)^{2}\right]
$$




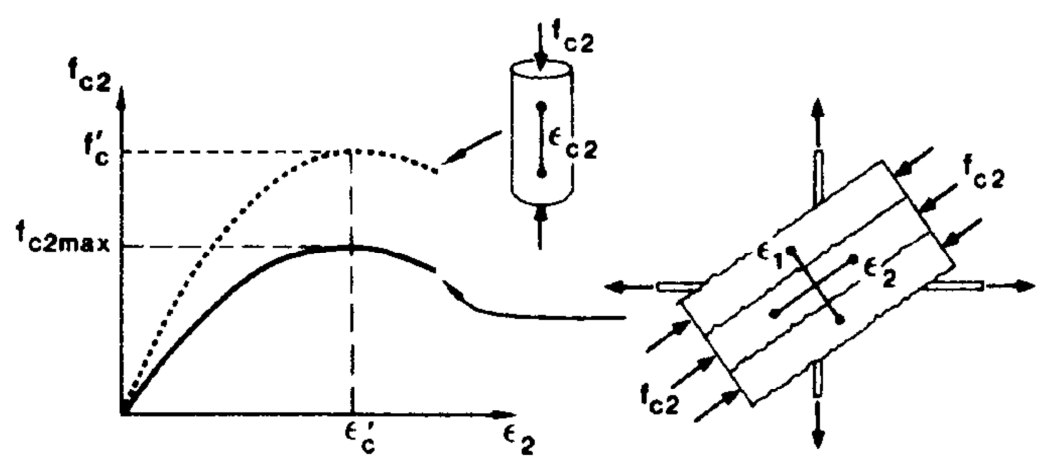

Figura 3.4 - Diagrama tensão-deformação para o concreto fissurado em compressão (VECCHIO E COLLINS, 1986)

Sendo assim, para este estudo é admitido que os valores definidos pela CEB (1990) para a resistência do concreto são seus limites máximo e mínimo e os valores intermediários serão interpolados pela eq. 3.2 e, assim, a resistência de pico do concreto fica determinada pela equação 3.5. Graficamente, a expressão é aquela mostrada na Figura 3.5.

$f_{c d 2} \leq f_{c 2 \max }=\frac{f_{c d 1}}{0,8+170 . \varepsilon_{1}} \leq f_{c d 1}$

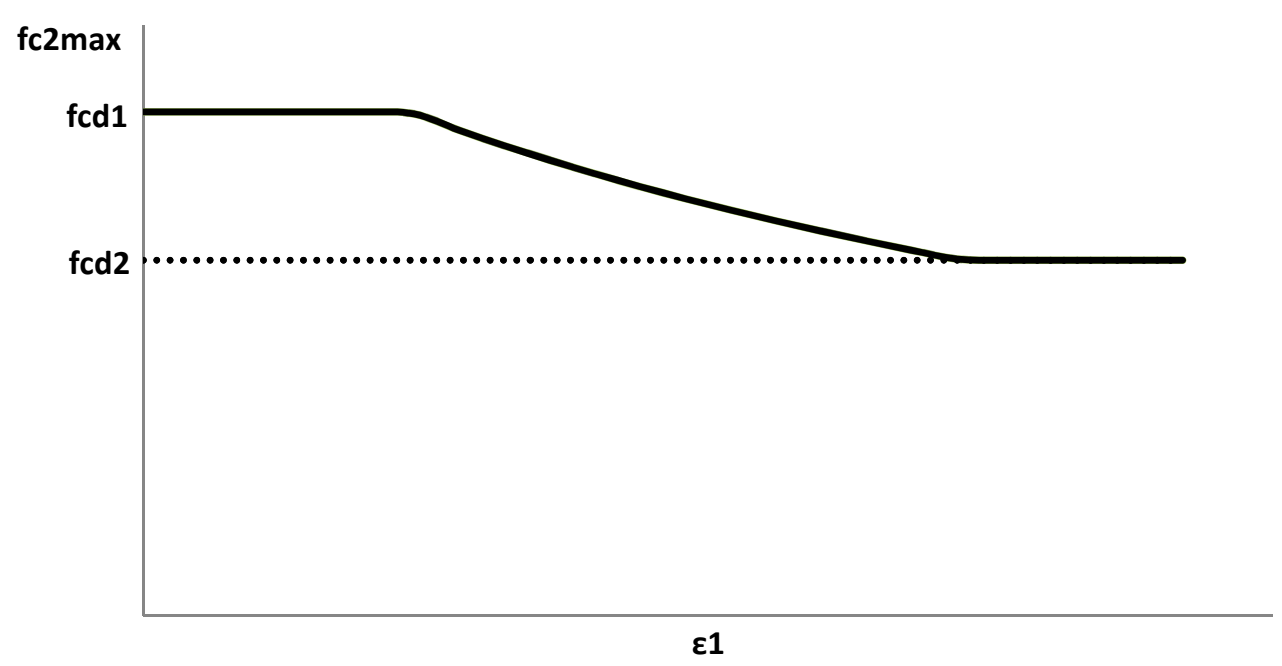

Figura 3.5 - Resistência máxima à compressão do concreto em função da deformação de tração perpendicular

Da mesma forma que no CEB (1990) e no trabalho de Vecchio e Collins (1986), a expressão do diagrama tensão-deformação adotada neste estudo será a equação 
3.4, porém se considerará a deformação de ruptura como aquela que correspondente a tensão de pico, valor que, conforme CEB (1990) é de $2 \%$, conforme a eq. 3.6 e a Figura 3.6.

$\varepsilon_{c 1}=\varepsilon_{c}^{\prime}=\varepsilon_{c u}=2 \%$

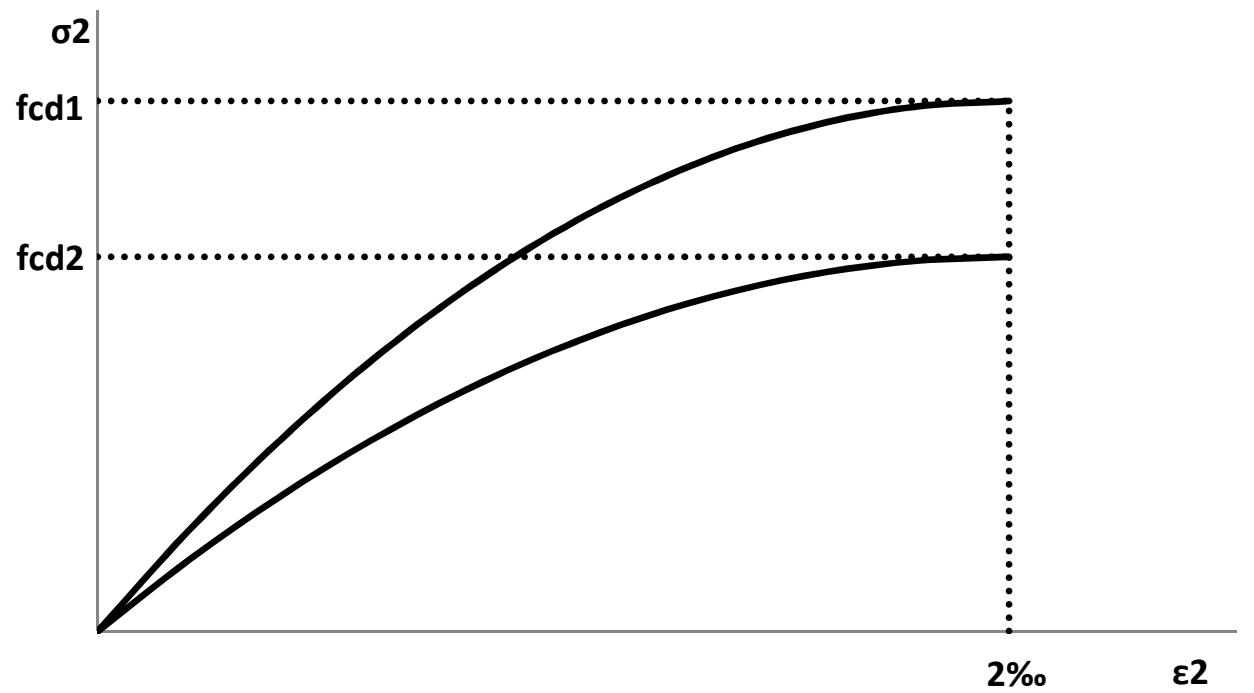

Figura 3.6 - Diagrama tensão-deformação

\subsection{Análise das deformações}

Seja um elemento de chapa com as deformações representadas na Figura 3.7, onde $\varepsilon_{1}$ e $\varepsilon_{2}$ são deformações principais e $\varepsilon_{x}$ e $\varepsilon_{y}$ são as deformações nas respectivas direções $x$ e y e $\gamma_{x y}$ é a distorção no plano xy. 


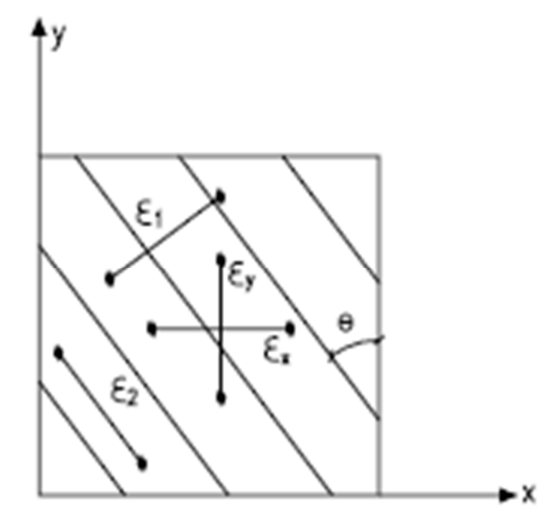

Figura 3.7 - Deformação no elemento de chapa (JAZRA, 2008)

As deformações quando de tração terão sinal positivo, consequentemente, quando de compressão terão sinal negativo. A distorção assume valores positivos quando for como apresentado na Figura 3.8.

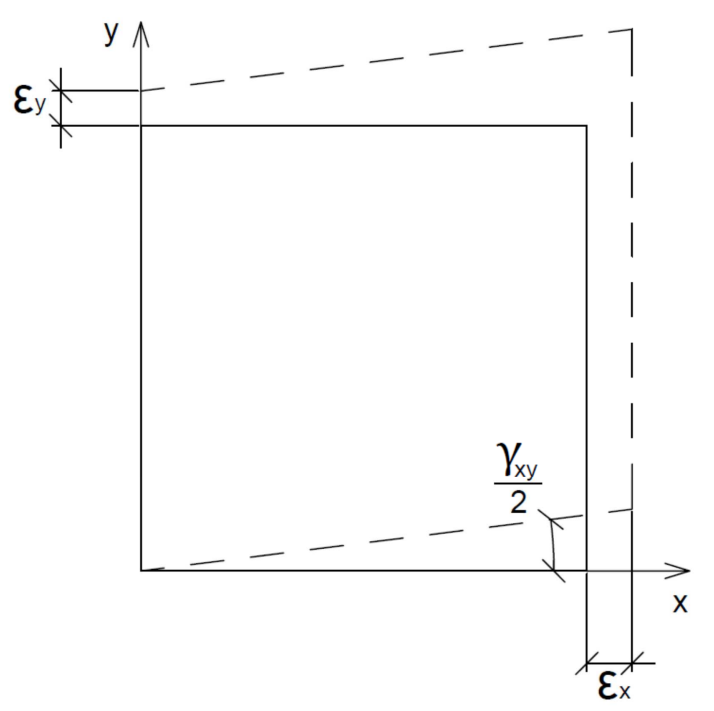

Figura 3.8 - Sentido positivo das deformações

Admitindo um conjunto de deformações qualquer, pode-se representa-lo através do círculo de Mohr, como apresentado na Figura 3.9. 


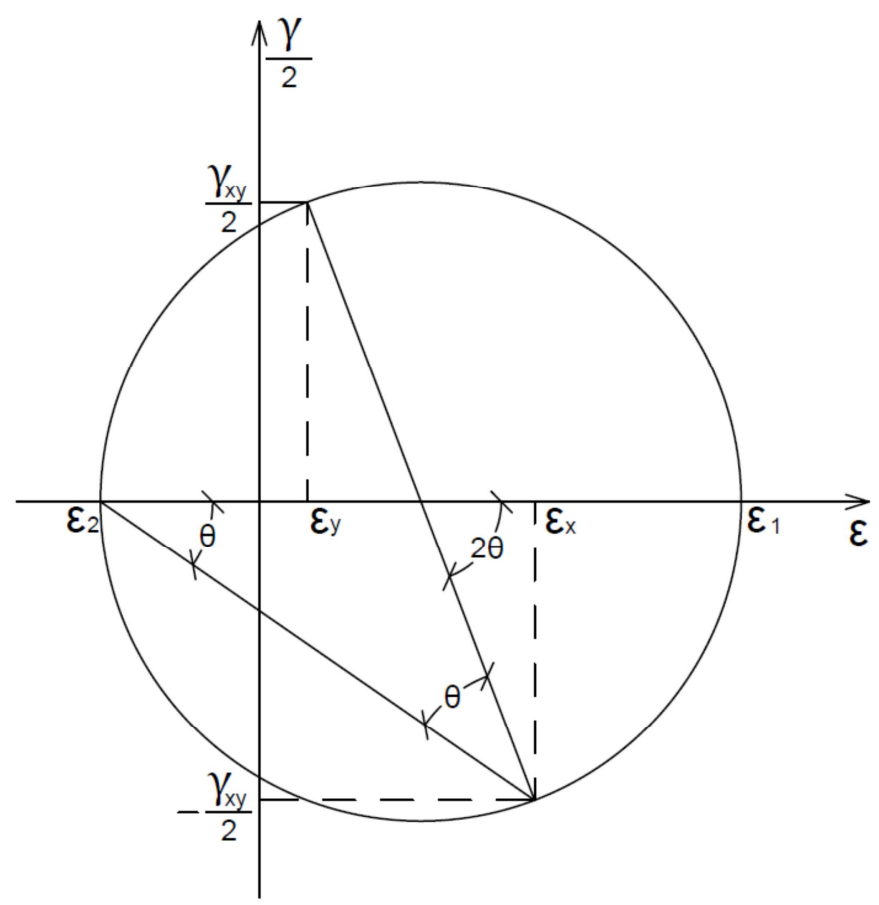

Figura 3.9 - Círculo de Mohr das deformações

Através do círculo de Mohr da Figura 3.9, podem-se inferir as seguintes relações:

Para determinar a distorção através das deformações $\varepsilon_{x}, \varepsilon_{y}$ e $\theta$ :

$\frac{\gamma_{x y}}{2}=\operatorname{tg} 2 \theta \cdot\left(\frac{\varepsilon_{x}-\varepsilon_{y}}{2}\right)$

Para determinar as deformações principais através de $\varepsilon_{x}, \varepsilon_{y}$ e $\gamma_{x y}$ :

$\varepsilon_{1}=\frac{\varepsilon_{x}+\varepsilon_{y}}{2}+\sqrt{\frac{\left(\varepsilon_{x}-\varepsilon_{y}\right)^{2}}{4}+\left(\frac{\gamma_{x y}}{2}\right)^{2}}$
$\varepsilon_{2}=\frac{\varepsilon_{x}+\varepsilon_{y}}{2}-\sqrt{\frac{\left(\varepsilon_{x}-\varepsilon_{y}\right)^{2}}{4}+\left(\frac{\gamma_{x y}}{2}\right)^{2}}$

Pode-se também determinar as deformações principais utilizando $\varepsilon_{x}, \varepsilon_{y}$ e $\theta$ :

$\varepsilon_{1}=\frac{\varepsilon_{x}+\varepsilon_{y}}{2}+\left(\frac{\varepsilon_{x}-\varepsilon_{y}}{2 \cdot \cos 2 \theta}\right)$ 
$\varepsilon_{2}=\frac{\varepsilon_{x}+\varepsilon_{y}}{2}-\left(\frac{\varepsilon_{x}-\varepsilon_{y}}{2 \cdot \cos 2 \theta}\right)$

Ao contrário, pode-se obter $\varepsilon_{\mathrm{x}}$ e $\varepsilon_{\mathrm{y}}$ através de $\varepsilon_{1}$, $\varepsilon_{2}$ e $\theta$ :

$\varepsilon_{x}=\frac{\varepsilon_{1}+\varepsilon_{2}}{2}+\left(\frac{\varepsilon_{1}-\varepsilon_{2}}{2}\right) \cdot \cos 2 \theta$

$\varepsilon_{y}=\frac{\varepsilon_{1}+\varepsilon_{2}}{2}-\left(\frac{\varepsilon_{1}-\varepsilon_{2}}{2}\right) \cdot \cos 2 \theta$

Também, do círculo de Mohr, tem-se que:

$\varepsilon_{1}+\varepsilon_{2}=\varepsilon_{x}+\varepsilon_{y}$

\subsection{Verificação da resistência à compressão do concreto}

Conforme visto no Capitulo 2, a resistência do concreto para o método baseado nos critérios de Baumann segue as recomendações do CEB (1990). Porém, neste estudo será utilizado o diagrama tensão-deformação apresentado no item 3.1. Isto altera a forma de verificar se a tensão de compressão atuante no concreto está abaixo de sua resistência.

Para o caso IV, a verificação do concreto é feita da mesma forma que apresentada no item 2.3.5.4, pois neste caso não há tração na chapa e a resistência à compressão do concreto é dada sempre por $\mathrm{f}_{\mathrm{cd} 1}$.

Já para os casos II e III, primeiramente deve-se verificar se:

$\sigma_{c}=\frac{n_{c}}{h}>f_{c d 1}$

Como se admite neste estudo que $f_{c d 1}$ é o valor máximo para a resistência do concreto à compressão em qualquer caso, se a inequação 3.15 for satisfeita concluise que a tensão de compressão no concreto não atende ao limite estabelecido, 
sendo assim, deve-se verificar a possibilidade de se utilizar armaduras que atuem a compressão.

Caso a inequação 3.15 não for atendida, verifica-se se:

$\sigma_{c}=\frac{n_{c}}{h} \leq f_{c d 2}$

Como $\mathrm{f}_{\mathrm{cd} 2}$ é o limite inferior para a resistência à compressão do concreto, se a expressão 3.16 for satisfeita conclui-se que a tensão de compressão no concreto atende ao limite de resistência imposto.

Se as inequações 3.15 e 3.16 não forem satisfeitas, por consequência tem-se que:

$f_{c d 2}<\frac{n_{c}}{h} \leq f_{c d 1}$

Neste caso, deve se analisar as deformações na chapa para determinar o limite de resistência a ser utilizado, pois ele dependerá de $\varepsilon_{1}$.

\subsubsection{Cálculo para determinação do limite de resistência a compressão do concreto}

Neste problema tem-se como objetivo encontrar o valor de $f_{c d 2 m a x}$. Porém, ele depende da deformação da chapa. Será apresentada uma forma de cálculo baseada naquela apresentada por Jazra (2008). Este cálculo é válido para os casos II e III. Como eles são análogos tendo como diferença apenas o posicionamento da armadura (eixo y para caso II e eixo x para caso III), será descrito somente aquele para o caso III, sendo que para o caso II será necessário substituir a equação 3.12 pela equação 3.13 e repetir a mesmo processo.

Sendo assim, dado que:

$\varepsilon_{x}=\frac{\varepsilon_{1}+\varepsilon_{2}}{2}+\left(\frac{\varepsilon_{1}-\varepsilon_{2}}{2}\right) \cdot \cos 2 \theta$

Então: 
2. $\varepsilon_{x}=\varepsilon_{1}+\varepsilon_{2}+\left(\varepsilon_{1}-\varepsilon_{2}\right) \cdot \cos 2 \theta$

2. $\varepsilon_{x}=\varepsilon_{1}+\varepsilon_{2}+\varepsilon_{1} \cdot \cos 2 \theta-\varepsilon_{2} \cdot \cos 2 \theta$

2. $\varepsilon_{x}=\varepsilon_{1} \cdot(1+\cos 2 \theta)+\varepsilon_{2} \cdot(1-\cos 2 \theta)$

$\varepsilon_{1} \cdot(1+\cos 2 \theta)=2 \cdot \varepsilon_{x}-\varepsilon_{2} \cdot(1-\cos 2 \theta)$

$\varepsilon_{1}=\frac{2 \cdot \varepsilon_{x}-\varepsilon_{2} \cdot(1-\cos 2 \theta)}{(1+\cos 2 \theta)}$

Retomando a expressão 3.5 .

$f_{c d 2} \leq f_{c 2 \max }=\frac{f_{c d 1}}{0,8+170 . \varepsilon_{1}} \leq f_{c d 1}$

$f_{c 2 \max }=\frac{f_{c d 1}}{0,8+170 \cdot \varepsilon_{1}}$

Substituindo 3.18 em 3.5, tem-se que:

$f_{c 2 \max }=\frac{f_{c d 1}}{0,8+170 \cdot\left[\frac{2 \cdot \varepsilon_{x}-\varepsilon_{2} \cdot(1-\cos 2 \theta)}{(1+\cos 2 \theta)}\right]}$

Retomando a expressão 3.4

$\sigma_{c}=f_{c 2 \max } \cdot\left[2 \cdot\left(\frac{\varepsilon_{2}}{\varepsilon_{c}^{\prime}}\right)-\left(\frac{\varepsilon_{2}}{\varepsilon_{c}^{\prime}}\right)^{2}\right]$

As raízes da equação 3.4 são:

$\varepsilon_{2}=\varepsilon_{c}^{\prime} \cdot\left(1 \pm \sqrt{1-\frac{\sigma_{c}}{f_{c 2 \max }}}\right)$

Somente a solução com sinal negativo faz parte do domínio do problema, pois aquela com sinal positivo resulta em deformações maiores que $\varepsilon^{\prime}$. Então, substituindo 3.17 em 3.18 e lembrando que neste estudo $\varepsilon^{\prime}{ }_{c}=-2 \%$, tem-se que: 
$\varepsilon_{2}=-2 \% 0 \cdot\left(1-\sqrt{1-\frac{\sigma_{c}}{\frac{f_{c d 1}}{0,8+170 \cdot\left[\frac{2 \cdot \varepsilon_{x}-\varepsilon_{2} \cdot(1-\cos 2 \theta)}{(1+\cos 2 \theta)}\right]}}}\right)$

Dado que os cálculos das armaduras apresentados no Capítulo 2 são feitos admitindo que no ELU elas estejam em escoamento, então, a partir do diagrama tensão-deformação do aço mostrado no item 2.3.4.1 tem-se que:

$\varepsilon_{y d} \leq \varepsilon_{x} \leq 10 \%$

Neste estudo, a deformação para as barras em escoamento será sempre igual a $\varepsilon_{y d}$, pois assim será obtida a menor deformação na chapa possível no ELU. Desta forma, a resistência limite do concreto será consequentemente otimizada.

Desta forma, a expressão 3.19 fica assim:

$\varepsilon_{2}=-2 \% 0 \cdot\left(1-\sqrt{1-\frac{\sigma_{c}}{\frac{f_{c d 1}}{0,8+170 \cdot\left[\frac{2 \cdot \varepsilon_{y d}-\varepsilon_{2} \cdot(1-\cos 2 \theta)}{(1+\cos 2 \theta)}\right]}}}\right)$

Como a partir do cálculo apresentado no item 2.3.5.3 obtém-se o valor de $\theta$ e de $\sigma_{\mathrm{c}}$, o valor de $\varepsilon_{2}$ fica determinado. A expressão para o caso II é:

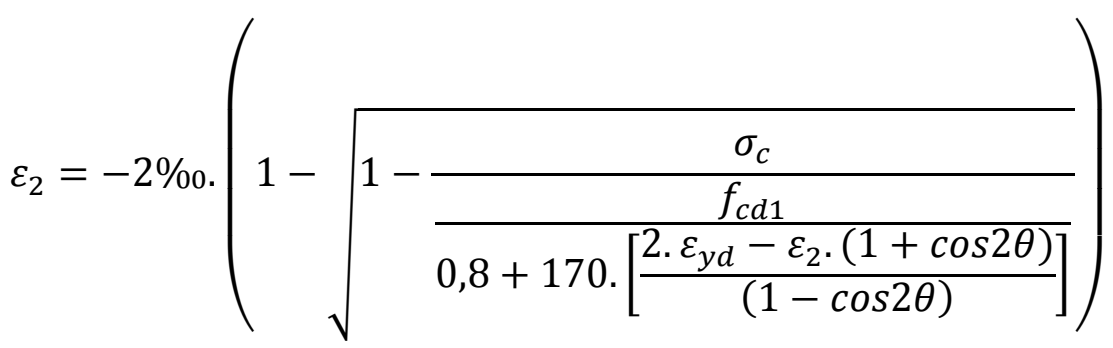


Tanto 3.20 como 3.21 são solucionáveis por métodos iterativos. Arbitrando-se um valor inicial para $\varepsilon_{2}$ para o qual a função exista, ou seja, de forma que o radicando não seja negativo, ela irá convergir para a solução do problema. Se em uma iteração o radicando assumir valor negativo, então a problema não tem solução e, portanto, a tensão no concreto está superior ao limite máximo. A inequação 3.22 mostra esse caso.

$\frac{\sigma_{c}}{\frac{f_{c d 1}}{0,8+170 \cdot\left[\frac{2 \cdot \varepsilon_{y d}-\varepsilon_{2} \cdot(1-\cos 2 \theta)}{(1+\cos 2 \theta)}\right]}}>1$

\subsubsection{Exemplos de dimensionamento}

Serão feitos quatro exemplos aplicando os conceitos até aqui expostos. Todos eles recaem no caso III de dimensionamento do CEB (1990). A resolução para o caso II é análoga. Em todos os exemplos deste estudo serão adotados os coeficientes de segurança recomendados pela NBR 6118. Todos os esforços solicitantes são de cálculo.

\subsubsection{Exemplo 3.1 - Tensão de compressão menor que $f_{c d 2}$}

Dado uma chapa com espessura de $12 \mathrm{~cm}$, $\mathrm{f}_{\mathrm{ck}}$ igual a $25 \mathrm{MPa}, \mathrm{CA}-50$ e submetida a esforços por unidade de comprimento como mostrados na Figura 3.10. 


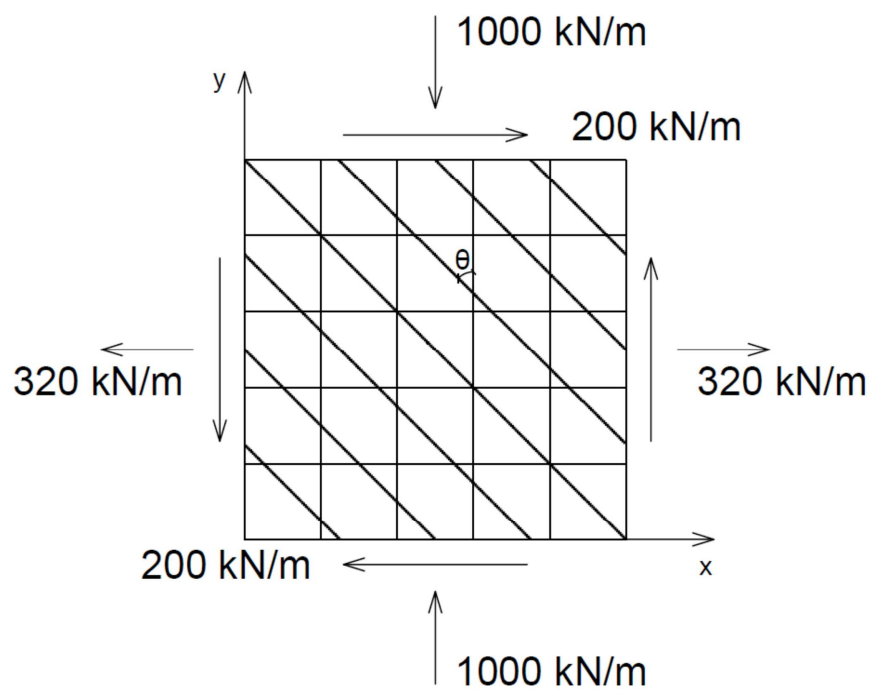

Figura 3.10 - Exemplo 3.1 - Tensão de compressão menor que $f_{c d 2}$

$h=12 \mathrm{~cm}$

$f_{c k}=25 \mathrm{MPa}$

$n_{x}=320 \mathrm{kN} / \mathrm{m}$

$n_{y}=-1000 \mathrm{kN} / \mathrm{m}$

$n_{x y}=200 \mathrm{kN} / \mathrm{m}$

Primeiramente, verifica-se que caso de dimensionamento se aplica para esta chapa calculando os esforços $n_{s x}$ e $n_{\text {sy }}$ através das expressões 2.11 e 2.12

$n_{s x}=n_{x}+\left|n_{x y}\right|$

$n_{s y}=n_{y}+\left|n_{x y}\right|$

Então, tem-se que:

$$
\begin{aligned}
& n_{s x}=n_{x}+\left|n_{x y}\right|=320+|200|=500 \mathrm{kN} / \mathrm{m} \\
& n_{s y}=n_{y}+\left|n_{x y}\right|=-1000+|200|=-800 \mathrm{kN} / \mathrm{m}
\end{aligned}
$$

Como $n_{s x}>0$ e $n_{s y} \leq 0$, pode-se dispensar o uso de armaduras de tração na direção y. Isto caracteriza o caso III de dimensionamento. Assim, primeiramente verifica-se o concreto: 


$$
\begin{aligned}
& \theta=\operatorname{arctg}\left(-\frac{n_{x y}}{n_{y}}\right)=\operatorname{arctg}\left(-\frac{200}{-1000}\right)=11,31^{\circ} \\
& n_{c}=n_{x y} \cdot(\operatorname{tg} \theta+\operatorname{cotg} \theta) \\
& n_{c}=200 \cdot\left(\operatorname{tg} 13,31^{\circ}+\operatorname{cotg} 13 \cdot 31^{\circ}\right) \\
& n_{c}=1040 \mathrm{kN} / \mathrm{m} \\
& \sigma_{c}=\frac{n_{c}}{h}=\frac{1040}{0,12}=8666,67 \frac{k N}{m^{2}}=8,67 \mathrm{MPa} \\
& f_{c d 1}=0,85 \cdot\left(1-\frac{f_{c k}}{250}\right) \cdot f_{c d}=0,85 \cdot\left(1-\frac{25}{250}\right) \cdot \frac{25}{1,4}=13,66 \mathrm{MPa} \\
& f_{c d 2}=0,60 \cdot\left(1-\frac{f_{c k}}{250}\right) \cdot f_{c d}=0,60 \cdot\left(1-\frac{f_{c k}}{250}\right) \cdot \frac{25}{1,4}=9,64 \mathrm{MPa}
\end{aligned}
$$

Como neste caso $\sigma_{c}<f_{c d 2}$, a tensão de compressão no concreto está abaixo do limite mínimo para a resistência do concreto e, portanto, satisfaz a condição de segurança. Sendo assim, pode-se calcular as armaduras de tração.

$$
\begin{aligned}
& n_{s x}=n_{x}-\frac{n_{x y}{ }^{2}}{n_{y}}=320-\frac{200^{2}}{-1000}=360 \mathrm{kN} / \mathrm{m} \\
& a_{s x}=\frac{n_{s x}}{f_{y d}}=\frac{360}{\frac{50}{1,15}}=8,28 \mathrm{~cm}^{2} / \mathrm{m} \\
& a_{s y}=0
\end{aligned}
$$

\subsubsection{Exemplo 3.2 - Tensão de compressão entre $f_{c d 1}$ e $f_{c d 2}$ e abaixo de $f_{c 2 \max }$}

Este exemplo é semelhante ao exemplo 3.1, porém o esforço de cisalhamento desta vez é um pouco maior, sendo que a tensão no concreto ficará entre $f_{c d 1}$ e $f_{c d 2}$. Dada a chapa apresentada na Figura 3.11. 


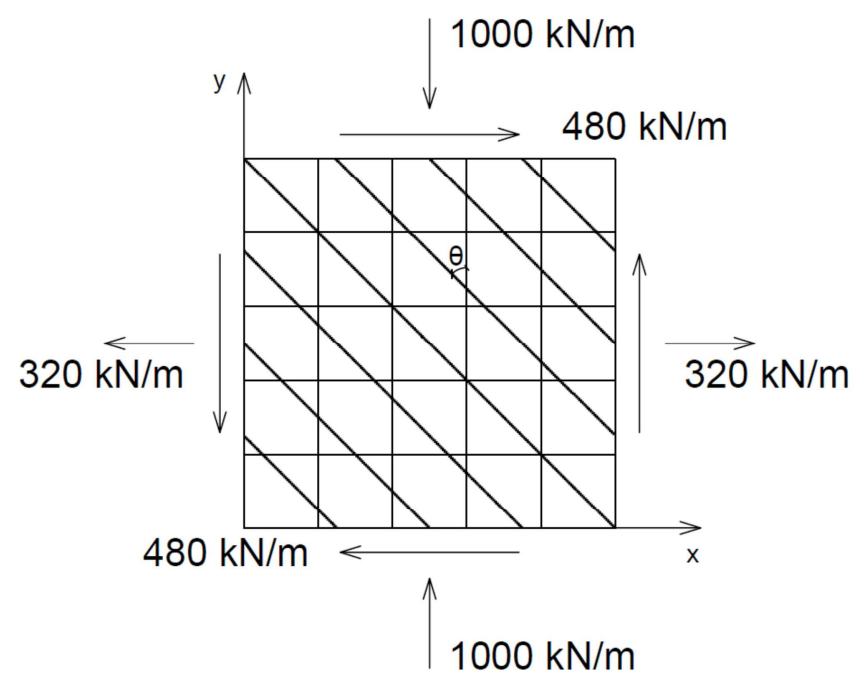

Figura 3.11 - Exemplo 3.2 - Tensão de compressão entre $f_{c d 1}$ e $f_{c d 2}$ e abaixo de $f_{c 2 m a x}$

$h=12 \mathrm{~cm}$

$f_{c k}=25 M P a$

$n_{x}=320 \mathrm{kN} / \mathrm{m}$

$n_{y}=-1000 \mathrm{kN} / \mathrm{m}$

$n_{x y}=480 \mathrm{kN} / \mathrm{m}$

Da mesma forma que no exemplo 3.1, primeiramente verifica-se em qual caso de dimensionamento essa chapa se encaixa.

$n_{s x}=n_{x}+\left|n_{x y}\right|=320+|480|=800 \mathrm{kN} / \mathrm{m}$

$n_{s y}=n_{y}+\left|n_{x y}\right|=-1000+|480|=-520 \mathrm{kN} / \mathrm{m}$

Assim como no exemplo 3.1, $\mathrm{n}_{\mathrm{sx}}>0$ e $\mathrm{n}_{\mathrm{sy}} \leq 0$, então pode-se dispensar o uso de armaduras de tração na direção y, o que caracteriza o caso III de dimensionamento. Assim, primeiramente verifica-se o concreto:

$$
\begin{aligned}
& \theta=\operatorname{arctg}\left(-\frac{n_{x y}}{n_{y}}\right)=\operatorname{arctg}\left(-\frac{480}{-1000}\right)=25,64^{\circ} \\
& n_{c}=n_{x y} \cdot(\operatorname{tg} \theta+\operatorname{cotg} \theta) \\
& n_{c}=480 \cdot\left(\operatorname{tg} 25,64^{\circ}+\operatorname{cotg} 25,64^{\circ}\right) \\
& n_{c}=1230,4 \mathrm{kN} / \mathrm{m}
\end{aligned}
$$


$\sigma_{c}=\frac{n_{c}}{h}=\frac{1230,4}{0,12}=10253,33 \frac{k N}{m^{2}}=10,25 \mathrm{MPa}$

$f_{c d 1}=0,85 \cdot\left(1-\frac{f_{c k}}{250}\right) \cdot f_{c d}=0,85 \cdot\left(1-\frac{25}{250}\right) \cdot \frac{25}{1,4}=13,66 \mathrm{MPa}$

$f_{c d 2}=0,60 \cdot\left(1-\frac{f_{c k}}{250}\right) \cdot f_{c d}=0,60 \cdot\left(1-\frac{f_{c k}}{250}\right) \cdot \frac{25}{1,4}=9,64 \mathrm{MPa}$

Neste caso $\mathrm{f}_{\mathrm{cd} 2}<\sigma_{\mathrm{c}}<\mathrm{f}_{\mathrm{cd} 1}$, então se deve achar o valor de $\mathrm{f}_{\mathrm{c} 2 \mathrm{max}}$ utilizando $\mathrm{o}$ procedimento apresentado no item 3.3.1. Assim, dada a expressão 3.20 tem-se que:

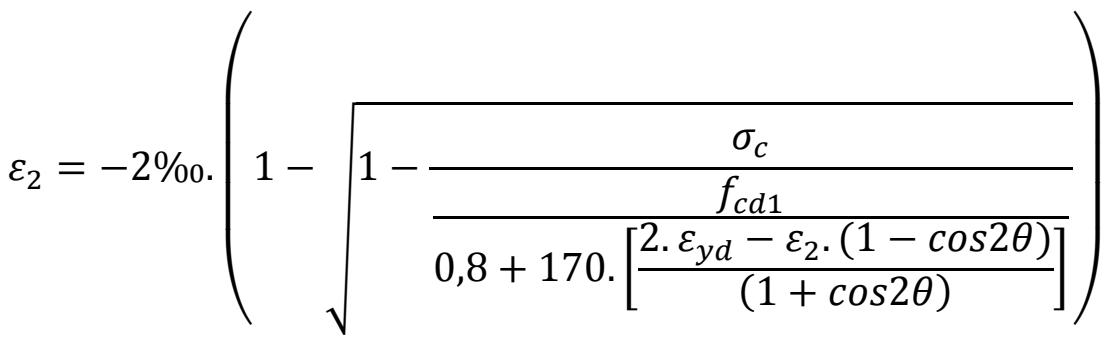

$\varepsilon_{y d}=\frac{f_{y d}}{E_{c s}}=\frac{\frac{500}{1,15}}{210000}=2,07 \% 0$

Substituindo os valores já calculados em 3.20, tem-que:

$\varepsilon_{2}=-2 \% \cdot\left(1-\sqrt{\left.1-\frac{10,25}{\frac{13,66}{0,8+170 \cdot\left[\frac{2.2,07 \% 0-\varepsilon_{2} \cdot\left(1-\cos \left(2.25 .64^{\circ}\right)\right)}{\left(1+\cos \left(2.25 .64^{\circ}\right)\right)}\right]}}\right)}\right)$

Então, arbitrando um $\varepsilon_{2}$ para o qual a função exista, iterativamente é possível achar o valor de $\varepsilon_{2}$ para este caso, conforme mostrado na Tabela 3.1.

Tabela 3.1 - Cálculo iterativo de $\mathrm{f}_{\mathrm{c} 2 \max }$ para o exemplo 3.2

\begin{tabular}{|c|c|c|c|c|c|}
\hline $\mathrm{i}$ & $\varepsilon_{2 \mathrm{i}}(\%)$ & $\varepsilon_{1}(\%)$ & $\mathrm{f}_{\mathrm{c} 2 \max }(\mathrm{MPa})$ & Radicando & $\varepsilon_{2 \mathrm{f}}(\%)$ \\
\hline 1 & 0,000 & 2,547 & 11,079 & 0,075 & $-1,454$ \\
\hline 2 & $-1,454$ & 2,882 & 10,590 & 0,032 & $-1,644$ \\
\hline 3 & $-1,644$ & 2,926 & 10,529 & 0,026 & $-1,676$ \\
\hline 4 & $-1,676$ & 2,934 & 10,519 & 0,025 & $-1,682$ \\
\hline 5 & $-1,682$ & 2,935 & 10,517 & 0,025 & $-1,684$ \\
\hline
\end{tabular}




\begin{tabular}{|l|l|l|l|l|l|}
6 & $-1,684$ & 2,935 & 10,516 & 0,025 & $-1,684$ \\
\hline 7 & $-1,684$ & 2,935 & 10,516 & 0,025 & $-1,684$ \\
\hline 8 & $-1,684$ & 2,935 & 10,516 & 0,025 & $-1,684$ \\
\hline
\end{tabular}

Portanto, tem-se que:

$\varepsilon_{2}=-1,684 \%$

$\varepsilon_{1}=2,935 \%$

$f_{c 2 \max }=10,516 \mathrm{MPa}>\sigma_{c}=10,25 \mathrm{MPa}$

Assim, o concreto esta submetido a uma tensão de compressão abaixo do limite. Então, calculam-se as armaduras de tração.

$$
\begin{aligned}
& n_{s x}=n_{x}-\frac{n_{x y}^{2}}{n_{y}}=320-\frac{480^{2}}{-1000}=550,4 \mathrm{kN} / \mathrm{m} \\
& a_{s x}=\frac{n_{s x}}{f_{y d}}=\frac{550,4}{\frac{50}{1,15}}=12,66 \mathrm{~cm}^{2} / \mathrm{m} \\
& a_{s y}=0
\end{aligned}
$$

\subsubsection{Exemplo 3.3 - Tensão de compressão entre $f_{c d 1}$ e $f_{c d 2}$ e acima de $f_{c 2 \max }$}

Neste exemplo o cisalhamento será maior que no exemplo 3.2, porém a tensão no concreto continuará entre $f_{c d 1}$ e $f_{c d 2}$. Dada a chapa apresentada na Figura 3.12. 


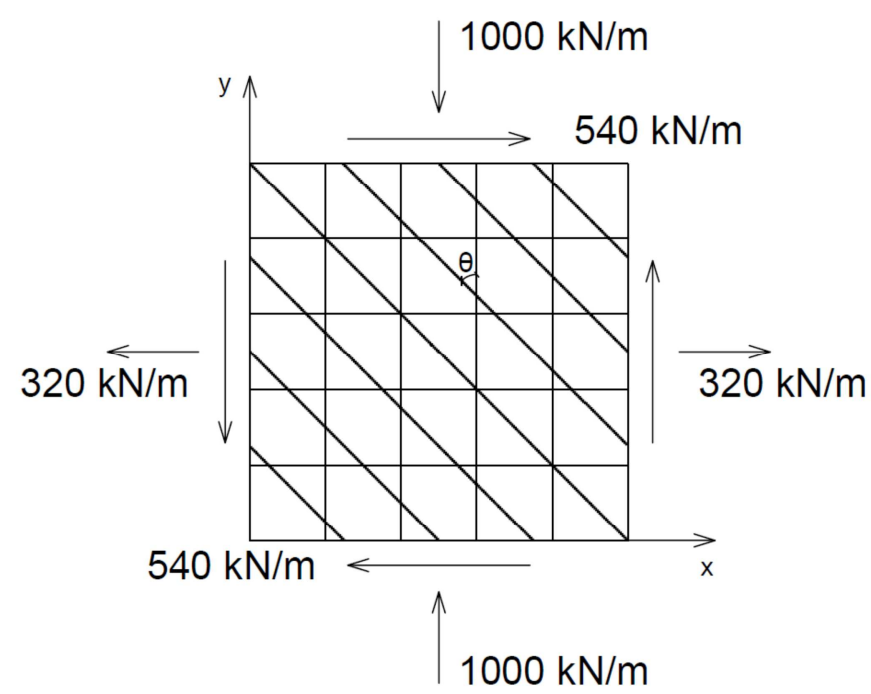

Figura 3.12 - Exemplo 3.3 - Tensão de compressão entre $f_{c d 1}$ e $f_{c d 2}$ e acima de $f_{c 2 m a x}$

$$
\begin{aligned}
& h=12 \mathrm{~cm} \\
& f_{c k}=25 \mathrm{MPa} \\
& n_{x}=320 \mathrm{kN} / \mathrm{m} \\
& n_{y}=-1000 \mathrm{kN} / \mathrm{m} \\
& n_{x y}=540 \mathrm{kN} / \mathrm{m}
\end{aligned}
$$

Da mesma forma que nos exemplos anteriores, primeiramente verifica-se em qual caso de dimensionamento essa chapa se encaixa.

$$
\begin{aligned}
& n_{s x}=n_{x}+\left|n_{x y}\right|=320+|540|=860 \mathrm{kN} / \mathrm{m} \\
& n_{s y}=n_{y}+\left|n_{x y}\right|=-1000+|540|=-460 \mathrm{kN} / \mathrm{m}
\end{aligned}
$$

Como nos outros exemplos, $\mathrm{n}_{\mathrm{sx}}>0$ e $\mathrm{n}_{\mathrm{sy}} \leq 0$, então pode-se dispensar o uso de armaduras de tração na direção y, caracterizando o caso III de dimensionamento. Então, seguindo o roteiro de cálculo, verifica-se o concreto:

$$
\begin{aligned}
& \theta=\operatorname{arctg}\left(-\frac{n_{x y}}{n_{y}}\right)=\operatorname{arctg}\left(-\frac{540}{-1000}\right)=28,37^{\circ} \\
& n_{c}=n_{x y} \cdot(\operatorname{tg} \theta+\operatorname{cotg} \theta) \\
& n_{c}=540 \cdot\left(\operatorname{tg} 28,37^{\circ}+\operatorname{cotg} 28,37^{\circ}\right) \\
& n_{c}=1291,6 \mathrm{kN} / \mathrm{m}
\end{aligned}
$$


$\sigma_{c}=\frac{n_{c}}{h}=\frac{1291,6}{0,12}=10763,33 \frac{\mathrm{kN}}{\mathrm{m}^{2}}=10,76 \mathrm{MPa}$

$f_{c d 1}=0,85 \cdot\left(1-\frac{f_{c k}}{250}\right) \cdot f_{c d}=0,85 \cdot\left(1-\frac{25}{250}\right) \cdot \frac{25}{1,4}=13,66 \mathrm{MPa}$

$f_{c d 2}=0,60 \cdot\left(1-\frac{f_{c k}}{250}\right) \cdot f_{c d}=0,60 \cdot\left(1-\frac{f_{c k}}{250}\right) \cdot \frac{25}{1,4}=9,64 \mathrm{MPa}$

Como no exemplo 2, $\mathrm{f}_{\mathrm{cd} 2}<\sigma_{\mathrm{c}}<\mathrm{f}_{\mathrm{cd} 1}$, então se deve achar o valor de $\mathrm{f}_{\mathrm{c} 2 \mathrm{max}}$ utilizando $\mathrm{o}$ procedimento apresentado no item 3.3.1. Assim, dada a expressão $3.20 \mathrm{e}$ substituindo os valores já calculados, tem-se que:

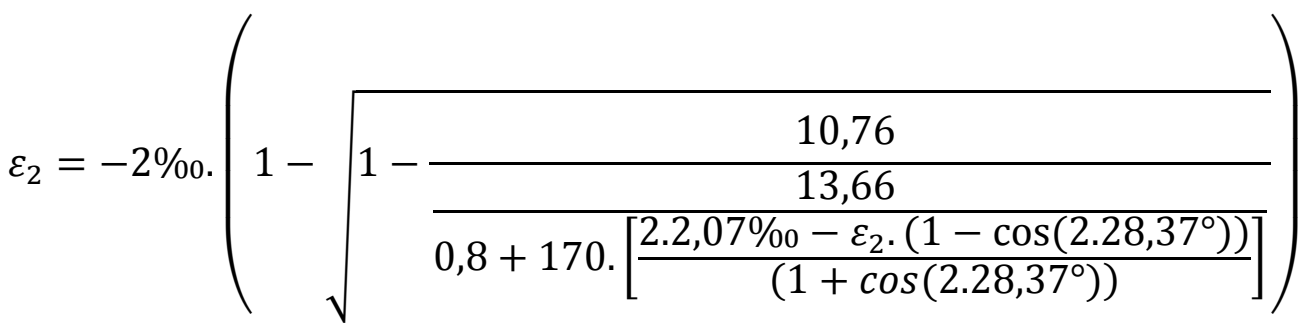

Então, arbitrando um $\varepsilon_{2}$ para o qual a função exista, iterativamente é possível achar $\circ$ valor de $\varepsilon_{2}$ para este caso. Neste caso, como o radicando dessa função acaba resultando negativo durante o processo iterativo, conforme mostrado na Tabela 3.2, a tensão no concreto está acima do limite imposto para um determinado $\varepsilon_{2}$.

Tabela 3.2 - Cálculo iterativo de $\mathrm{f}_{\mathrm{c} 2 \mathrm{max}}$ para o exemplo 3.3

\begin{tabular}{|c|c|c|c|c|c|}
\hline $\mathrm{i}$ & $\varepsilon_{2 \mathrm{i}}(\% \mathrm{o})$ & $\varepsilon_{1}(\%)$ & $\mathrm{f}_{\mathrm{c} 2 \max }(\mathrm{MPa})$ & Radicando & $\varepsilon_{2 \mathrm{f}}(\% \mathrm{o})$ \\
\hline 1 & 0,000 & 2,674 & 10,888 & 0,011 & $-1,786$ \\
\hline 2 & $-1,786$ & 3,195 & 10,171 & $-0,058$ & NÃO EXISTE \\
\hline
\end{tabular}

\subsubsection{Exemplo 3.4 - Tensão de compressão maior que $f_{c d 1}$}

Aumentando ainda mais a tensão de cisalhamento, a tensão no concreto será maior que $f_{c d 1}$. Dada a chapa apresentada na Figura 3.13. 


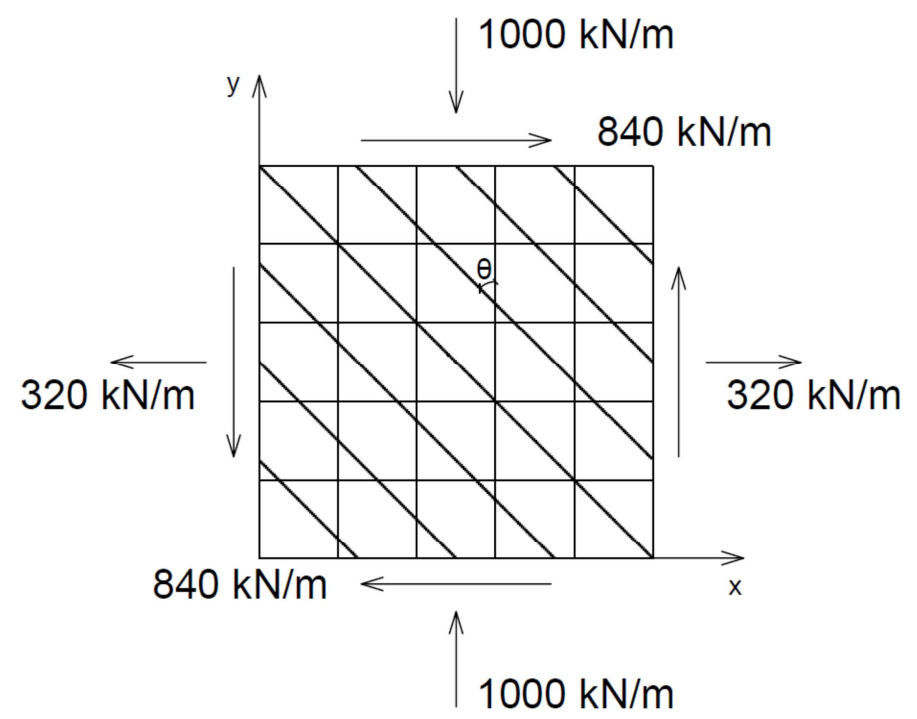

Figura 3.13 - Exemplo 3.4 - Tensão de compressão maior que $\mathrm{f}_{\mathrm{cd} 1}$

$h=12 \mathrm{~cm}$

$f_{c k}=25 \mathrm{MPa}$

$n_{x}=320 \mathrm{kN} / \mathrm{m}$

$n_{y}=-1000 \mathrm{kN} / \mathrm{m}$

$n_{x y}=840 \mathrm{kN} / \mathrm{m}$

Da mesma forma que nos exemplos anteriores, primeiramente verifica-se em qual caso de dimensionamento essa chapa se encaixa.

$n_{s x}=n_{x}+\left|n_{x y}\right|=320+|840|=1160 \mathrm{kN} / \mathrm{m}$
$n_{s y}=n_{y}+\left|n_{x y}\right|=-1000+|840|=-160 \mathrm{kN} / \mathrm{m}$

Como $n_{s x}>0$ e $n_{s y} \leq 0$, pode-se dispensar o uso de armaduras de tração na direção y, caracterizando o caso III de dimensionamento. Assim, verifica-se o concreto:

$$
\begin{aligned}
& \theta=\operatorname{arctg}\left(-\frac{n_{x y}}{n_{y}}\right)=\operatorname{arctg}\left(-\frac{840}{-1000}\right)=40,03^{\circ} \\
& n_{c}=n_{x y \cdot} \cdot(\operatorname{tg} \theta+\operatorname{cotg} \theta) \\
& n_{c}=840 \cdot\left(\operatorname{tg} 40,03^{\circ}+\operatorname{cotg} 40,03^{\circ}\right) \\
& n_{c}=1705,60 \mathrm{kN} / \mathrm{m}
\end{aligned}
$$




$$
\begin{aligned}
& \sigma_{c}=\frac{n_{c}}{h}=\frac{1040}{0,12}=8666,67 \frac{k N}{m^{2}}=14,21 \mathrm{MPa} \\
& f_{c d 1}=0,85 \cdot\left(1-\frac{f_{c k}}{250}\right) \cdot f_{c d}=0,85 \cdot\left(1-\frac{25}{250}\right) \cdot \frac{25}{1,4}=13,66 \mathrm{MPa} \\
& f_{c d 2}=0,60 \cdot\left(1-\frac{f_{c k}}{250}\right) \cdot f_{c d}=0,60 \cdot\left(1-\frac{f_{c k}}{250}\right) \cdot \frac{25}{1,4}=9,64 \mathrm{MPa}
\end{aligned}
$$

Como neste caso $\sigma_{c}>f_{c d 1}$, a tensão de compressão no concreto está acima do limite máximo, portanto não passa no critério de dimensionamento imposto. 


\section{DIMENSIONAMENTO DAS ARMADURAS DE COMPRESSÃO PARA OS CASOS II E III}

Assim como no Capítulo 3, todas as demonstrações deste capítulo serão feitas apenas para o caso III de dimensionamento. O caso II é análogo e somente será apresentada a sua formulação final.

Retomando o objetivo do trabalho, deseja-se obter critérios de dimensionamento para adoção de armaduras para quando a tensão de compressão no concreto for maior que sua resistência. A forma de avaliar este critério foi apresentada no Capítulo 3.

Primeiramente, as hipóteses do problema serão apresentadas. Na sequência serão apresentados os limites desse problema, ou seja, mostrar para quais casos é possível adotar armaduras de compressão.

\subsection{Hipóteses do problema}

Serão pressupostas para este problema as mesmas hipóteses dadas pelo método baseado nos critérios de Baumann apresentadas no item 2.3.

Além disso, algumas considerações sobre as deformações devem ser feitas. Primeiramente, assim como apresentado no item 3.3.1, será admitido que a deformação na direção $x$ é igual à deformação de escoamento da armadura de tração. Esta hipótese restringe a deformação na chapa, otimizando a resistência à compressão do concreto, além de resultar em uma área de armadura para a qual a chapa no ELU sofre ruptura dúctil. Em outras palavras, mesmo que para solucionar o problema seja necessário superarmar a chapa, este resultado será descartado, pois desta forma a chapa romperia de forma frágil.

Além disso, será considerado que a deformação $\varepsilon_{2}$ é sempre igual a $\varepsilon_{c}^{\prime}$, levando 0 concreto ao seu limite de resistência e, por consequência, reduzindo o consumo de armaduras. Resumindo as hipóteses, tem-se que: 
1. As fissuras apresentadas pelo elemento são aproximadamente paralelas e retilíneas.

2. A resistência à tração do concreto é desprezada.

3. O efeito de pino das armaduras é desprezado.

4. O efeito de engrenamento dos agregados é desprezado.

5. É considerada perfeita aderência entre a armadura e o concreto.

6. É desconsiderado o efeito devido ao "tension-stiffening".

7. A direção das deformações principais coincide com a direção das tensões principais.

8. A deformação na direção x é igual à deformação de escoamento da armadura de tração $\left(\varepsilon_{\mathrm{x}}=\varepsilon_{\mathrm{yd}}\right)$.

9. A deformação na direção principal de compressão é igual à deformação que resulta na tensão de pico do concreto $\left(\varepsilon_{2}=\varepsilon_{\mathrm{c}}^{\prime}\right)$.

\subsection{Limites de dimensionamento}

Dada as hipóteses apresentadas, pretende-se determinar os casos em que é possível dimensionar as armaduras de compressão. Assim, primeiramente, dada uma chapa que está submetida a esforços tais que pode-se dispensar o uso de armaduras de tração na direção y, portanto está no caso de dimensionamento III, e que a tensão de compressão no concreto é maior que 0 a resistência $f_{c 2 m a x}$, conforme apresentado no Capítulo 3, tem-se que:

$R_{d} \geq S_{d}$

A resistência é dada pela equação 3.17 .

$$
R_{d}=f_{c 2 \max }=\frac{f_{c d 1}}{0,8+170 \cdot\left[\frac{2 \cdot \varepsilon_{x}-\varepsilon_{2} \cdot(1-\cos 2 \theta)}{(1+\cos 2 \theta)}\right]}
$$


Dado que por hipótese $\varepsilon_{\mathrm{x}}=\varepsilon_{\mathrm{yd}}$ e $\varepsilon_{2}=\varepsilon_{\mathrm{c}}^{\prime}=-2 \%$ tem-se que:

$$
f_{c 2 \max }=\frac{f_{c d 1}}{0,8+170 \cdot\left[\frac{2 \cdot \varepsilon_{y d}+2 \% 0 .(1-\cos 2 \theta)}{(1+\cos 2 \theta)}\right]}
$$

Sendo que:

$f_{c d 2} \leq f_{c 2 \max } \leq f_{c d 1}$

O gráfico que descreve a resistência em função de $\theta$ é apresentado na Figura 4.1. Para o caso III, todas as funções de $\theta$ tem domínio de $0 \leq \theta \leq\left|45^{\circ}\right|$. Para o caso II de dimensionamento $|45 \%| \leq \theta \leq \mid 90^{\circ} \circ$.

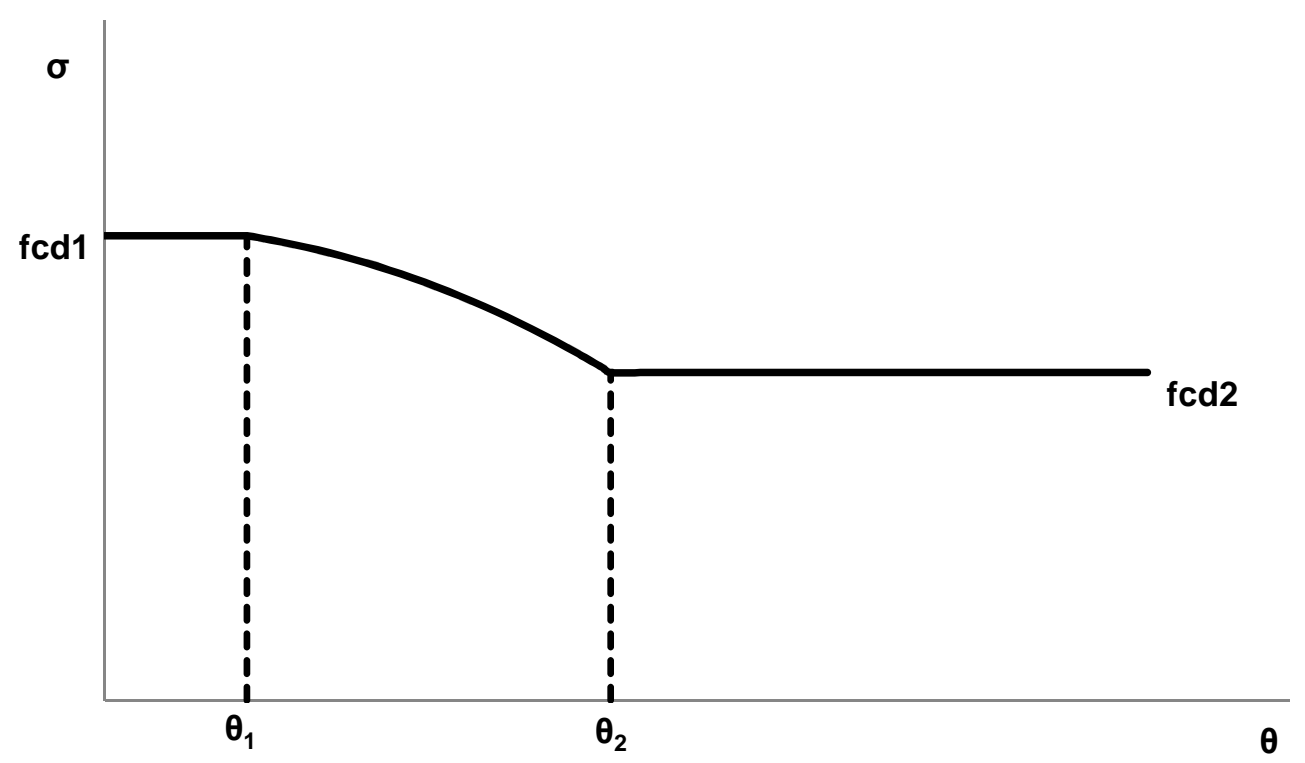

Figura 4.1 - Resistência à compressão do concreto em função de $\theta$ para o caso III

Pode-se determinar os valores de $\theta_{1}$ e $\theta_{2}$ apresentados na Figura 4.1 através das equações a seguir. $O$ ângulo $\theta_{1}$ é aquele que iguala $f_{c 2 m a x} a f_{c d 1}$. Desta forma tem-se que:

$\frac{f_{c d 1}}{0,8+170 \cdot\left[\frac{2 \cdot \varepsilon_{y d}+2 \% 0 \cdot\left(1-\cos 2 \theta_{1}\right)}{\left(1+\cos 2 \theta_{1}\right)}\right]}=f_{c d 1}$ 
$\frac{1}{0,8+170 \cdot\left[\frac{2 \cdot \varepsilon_{y d}+2 \% 0 \cdot\left(1-\cos 2 \theta_{1}\right)}{\left(1+\cos 2 \theta_{1}\right)}\right]}=1$

$0,8+170 \cdot\left[\frac{2 \cdot \varepsilon_{y d}+2 \% 0 \cdot\left(1-\cos 2 \theta_{1}\right)}{\left(1+\cos 2 \theta_{1}\right)}\right]=1$

$\frac{2 . \varepsilon_{y d}+2 \% 0 \cdot\left(1-\cos 2 \theta_{1}\right)}{\left(1+\cos 2 \theta_{1}\right)}=\frac{1-0,8}{170}$

2. $\varepsilon_{y d}+2 \%$ o. $\left(1-\cos 2 \theta_{1}\right)=0,00118 .\left(1+\cos 2 \theta_{1}\right)$

2. $\varepsilon_{y d}+2 \%-2 \% \cdot \cos 2 \theta_{1}=0,00118+0,00118 \cdot \cos 2 \theta_{1}$

$(-2 \%-0,00118) \cdot \cos 2 \theta_{1}=0,00118-2 \cdot \varepsilon_{y d}-2 \%$

$\cos 2 \theta_{1}=\frac{0,00118-2 \cdot \varepsilon_{y d}-2 \% 0}{(-2 \% 0-0,00118)}$

$\theta_{1}=\frac{\arccos \left(\frac{-0,82 \% 0-2 . \varepsilon_{y d}}{(-3,18 \% 0)}\right)}{2}$

Como a função cosseno produz o mesmo resultado não importa o sinal do ângulo, tanto $\theta_{1}$ positivo como negativo são soluções. De forma análoga, $\theta_{2}$ é o valor que iguala $\mathrm{f}_{\mathrm{c} 2 \mathrm{max}}$ com $\mathrm{f}_{\mathrm{cd} 2}$. Assim, tem-se que:

$\frac{f_{c d 1}}{0,8+170 \cdot\left[\frac{2 \cdot \varepsilon_{y d}+2 \% 0 \cdot\left(1-\cos 2 \theta_{2}\right)}{\left(1+\cos 2 \theta_{2}\right)}\right]}=f_{c d 2}$

$0,8+170 \cdot\left[\frac{2 \cdot \varepsilon_{y d}+2 \% 0 \cdot\left(1-\cos 2 \theta_{2}\right)}{\left(1+\cos 2 \theta_{2}\right)}\right]=\frac{f_{c d 1}}{f_{c d 2}}$

$\frac{2 . \varepsilon_{y d}+2 \% \mathrm{o}\left(1-\cos 2 \theta_{2}\right)}{\left(1+\cos 2 \theta_{2}\right)}=\frac{\frac{f_{c d 1}}{f_{c d 2}}-0,8}{170}$

Como:

$\frac{f_{c d 1}}{f_{c d 2}}=\frac{0,85 \cdot\left(1-\frac{f_{c k}}{250}\right) \cdot f_{c d}}{0,6 \cdot\left(1-\frac{f_{c k}}{250}\right) \cdot f_{c d}}=\frac{0,85}{0,60}=1,4167$

Então: 
$\frac{2 . \varepsilon_{y d}+2 \% 0 \cdot\left(1-\cos 2 \theta_{2}\right)}{\left(1+\cos 2 \theta_{2}\right)}=\frac{1,4167-0,8}{170}$

$\frac{2 . \varepsilon_{y d}+2 \% \cdot\left(1-\cos 2 \theta_{2}\right)}{\left(1+\cos 2 \theta_{2}\right)}=0,003627$

2. $\varepsilon_{y d}+2 \%$ o. $\left(1-\cos 2 \theta_{2}\right)=0,003627 \cdot\left(1+\cos 2 \theta_{2}\right)$

2. $\varepsilon_{y d}+2 \% 0-2 \%$ o. $\cos 2 \theta_{2}=0,003627+0,003627 \cdot \cos 2 \theta_{2}$

$(-2 \% 0-0,003627) \cdot \cos 2 \theta_{2}=0,003627-2 \cdot \varepsilon_{y d}-2 \% 0$

$\cos 2 \theta_{2}=\frac{0,003627-2 . \varepsilon_{y d}-2 \% 0}{(-2 \%-0,003627)}$

$\theta_{2}=\frac{\arccos \left(\frac{1,627 \% 0-2 . \varepsilon_{y d}}{(-5,627 \%)}\right)}{2}$

Também para $\theta_{2}$, tanto a solução positiva quanto negativa satisfazem a equação 4.3. Porém, se $\theta$ ultrapassar certo limite, a deformação $\varepsilon_{y}$ assume valores positivos. Desta forma, a área de armadura em y resulta em valores negativos, o que não é fisicamente possível. O exemplo do item 4.4.3 mostra esta situação. Dado que por hipótese $\varepsilon_{\mathrm{x}}=\varepsilon_{\mathrm{yd}}$ e que $\varepsilon_{2}=\varepsilon_{\mathrm{c}}^{\prime}$, pode-se calcular para quais valores de $\theta \varepsilon_{\mathrm{y}}$ é menor que 0 . Deseja-se então encontrar $\theta^{*}$ para o qual $\varepsilon_{y}=0$. Retomando a equação 3.14, tem-se que:

$\varepsilon_{1}+\varepsilon_{2}=\varepsilon_{x}+\varepsilon_{y}$

$\varepsilon_{1}=\varepsilon_{x}+\varepsilon_{y}-\varepsilon_{2}$

$\varepsilon_{1}=\varepsilon_{y d}+\varepsilon_{y}-\varepsilon_{c}^{\prime}$

Quando $\varepsilon_{y}=0$, tem-se que:

$\varepsilon_{1}=\varepsilon_{y d}-\varepsilon^{\prime}{ }_{c}$

Agora, retomando a equação 3.12, tem-se que:

$\varepsilon_{x}=\frac{\varepsilon_{1}+\varepsilon_{2}}{2}+\left(\frac{\varepsilon_{1}-\varepsilon_{2}}{2}\right) \cdot \cos 2 \theta^{*}$ 
$\cos 2 \theta^{*}=\frac{\varepsilon_{x}-\frac{\varepsilon_{1}+\varepsilon_{2}}{2}}{\left(\frac{\varepsilon_{1}-\varepsilon_{2}}{2}\right)}$

$\cos 2 \theta^{*}=\frac{\varepsilon_{y d}-\frac{\left(\varepsilon_{y d}+2 \% 0\right)-2 \% 0}{2}}{\left(\frac{\left(\varepsilon_{y d}+2 \% 0\right)+2 \% 0}{2}\right)}$

$\cos 2 \theta^{*}=\frac{\varepsilon_{y d}-\frac{\varepsilon_{y d}}{2}}{\left(\frac{\left(\varepsilon_{y d}+4 \% 0\right)}{2}\right)}$

$\cos 2 \theta^{*}=\frac{\frac{\varepsilon_{y d}}{2}}{\left(\frac{\left(\varepsilon_{y d}+4 \% 0\right)}{2}\right)}$

$\cos 2 \theta^{*}=\frac{\varepsilon_{y d}}{\varepsilon_{y d}+4 \% 0}$

Portanto:

$\theta^{*}=\frac{\arccos \left(\frac{\varepsilon_{y d}}{\varepsilon_{y d}+4 \% 0}\right)}{2}$

A Tabela 4.1 apresenta os valores de $\theta_{1}, \theta_{2}$ e $\theta^{*}$ para os aços determinados pela NBR 6118. Pode-se observar que para os aços CA-50 e CA-60 não existem valores de $\theta_{1}$. Isto acontece porque para os valores de deformação assumidos por hipótese para este problema, a resistência do concreto nunca atinge o valor de $f_{c d 1}$ para estes aços. Assim, a resistência do concreto atinge seu máximo para quando $\theta==0$.

Tabela 4.1 - Valores de $\theta_{1}, \theta_{2}$ e $\theta^{*}$ para os aços prescritos pela NBR 6118

\begin{tabular}{|c|c|c|c|c|}
\hline & $\varepsilon_{\text {yd }}(\% \circ)$ & $\left|\theta_{1}\right|\left(^{\circ}\right)$ & $\left|\theta_{2}\right|\left(^{(}\right)$ & $\left|\theta^{*}\right|\left(^{\circ}\right)$ \\
\hline CA-25 & 1,04 & 12,17 & 42,74 & 39,07 \\
\hline CA-50 & 2,07 & NÃO EXISTE & 31,74 & 35,03 \\
\hline CA-60 & 2,48 & NÃO EXISTE & 26,79 & 33,74 \\
\hline
\end{tabular}

Quanto à solicitação, retomando a equação 2.4, tem-se que: 
$S_{d}=\sigma_{c}=\frac{n_{c}}{h}=\frac{n_{x y}}{h} \cdot(\operatorname{tg} \theta+\operatorname{cotg} \theta)=\frac{2 \cdot n_{x y}}{h \cdot \operatorname{sen}(2 \theta)}$

Como:

$R_{d} \geq S_{d}$

Então, se $\theta_{1}$ existe e $\theta=0$, então $n_{x y}=0$ e:

$f_{c d 1}=\frac{n_{c}}{h}$

Se $\theta_{1}$ existe e $0<\theta \leq\left|\theta_{1}\right|$, então:

$f_{c d 1}=\frac{2 \cdot n_{x y}}{h \cdot \operatorname{sen}(2 \theta)}$

Se $\theta_{1}$ não existe e $\theta=0$, então $n_{x y}=0$ e:

$\frac{f_{c d 1}}{0,8+170 \cdot\left[\frac{2 \cdot \varepsilon_{y d}+2 \% 0 \cdot(1-\cos 2 \theta)}{(1+\cos 2 \theta)}\right]}=\frac{n_{c}}{h}$

Se $\theta_{1}$ existe e $\left|\theta_{1}\right|<\theta<\left|\theta_{2}\right|$ ou se $\theta_{1}$ não existe e $0<\theta<\left|\theta_{2}\right|$, então:

$\frac{f_{c d 1}}{0,8+170 \cdot\left[\frac{2 \cdot \varepsilon_{y d}+2 \% 0 \cdot(1-\cos 2 \theta)}{(1+\cos 2 \theta)}\right]}=\frac{2 \cdot n_{x y}}{h \cdot \operatorname{sen}(2 \theta)}$

$E$, finalmente, se $\left|\theta_{2}\right| \leq \theta<\left|\theta^{*}\right|$, então:

$f_{c d 2}=\frac{2 \cdot n_{x y}}{h \cdot \operatorname{sen}(2 \theta)}$

Dado este quadro, deseja-se saber para quais valores de esforços é possível dimensionar as armaduras de compressão. Para os esforços normais, não há limite 
matemático, havendo somente limite para a taxa geométrica de armadura prescrita pela NBR 6118. Já para o esforço de cisalhamento existe um limite cuja formulação varia de acordo com o tipo de aço adotado. Isto acontece porque os limites $\theta_{1}, \theta_{2}$ e $\theta^{*}$ são diferentes para cada aço, como mostrado na Tabela 4.1.

Para CA-25, como $\left|\theta^{*}\right|<\left|\theta_{2}\right|$, a equação 4.10 nunca será válida. Então, retomando a equação 4.9, deseja-se saber qual o máximo valor que $n_{x y}$ pode assumir.

$\frac{f_{c d 1}}{0,8+170 \cdot\left[\frac{2 \cdot \varepsilon_{y d}+2 \% 0 \cdot(1-\cos 2 \theta)}{(1+\cos 2 \theta)}\right]}=\frac{2 \cdot n_{x y}}{h \cdot \operatorname{sen}(2 \theta)}$

$n_{x y}=\frac{f_{c d 1} \cdot h}{2} \cdot\left(\frac{\operatorname{sen}(2 \theta)}{0,8+170 \cdot\left[\frac{2 \cdot \varepsilon_{y d}+2 \% 0 \cdot(1-\cos 2 \theta)}{(1+\cos 2 \theta)}\right]}\right)$

Para se obter o valor de $\theta$ que leva ao máximo da função de forma analítica é necessário deriva-la. Como o termo constante não se altera devido à derivação, pode-se estudar a função sem este termo.

$f(\theta)=\left(\frac{\operatorname{sen}(2 \theta)}{0,8+170 \cdot\left[\frac{2 \cdot \varepsilon_{y d}+2 \% 0 \cdot(1-\cos 2 \theta)}{(1+\cos 2 \theta)}\right]}\right)$

Assim, o gráfico dessa função é apresentado na Figura 4.2. Pode-se ver que para dado $f(\theta)$, podem existir dois valores de $\theta$ correspondentes. De forma a se evitar isso, o domínio da função será restrito entre $0 \leq\left|\theta_{x y}\right|$. As soluções maiores que $\left|\theta_{x y}\right|$ levam a um dimensionamento antieconômico, pois a deformação $\varepsilon_{y}$ diminui e o consumo de armadura aumenta. 


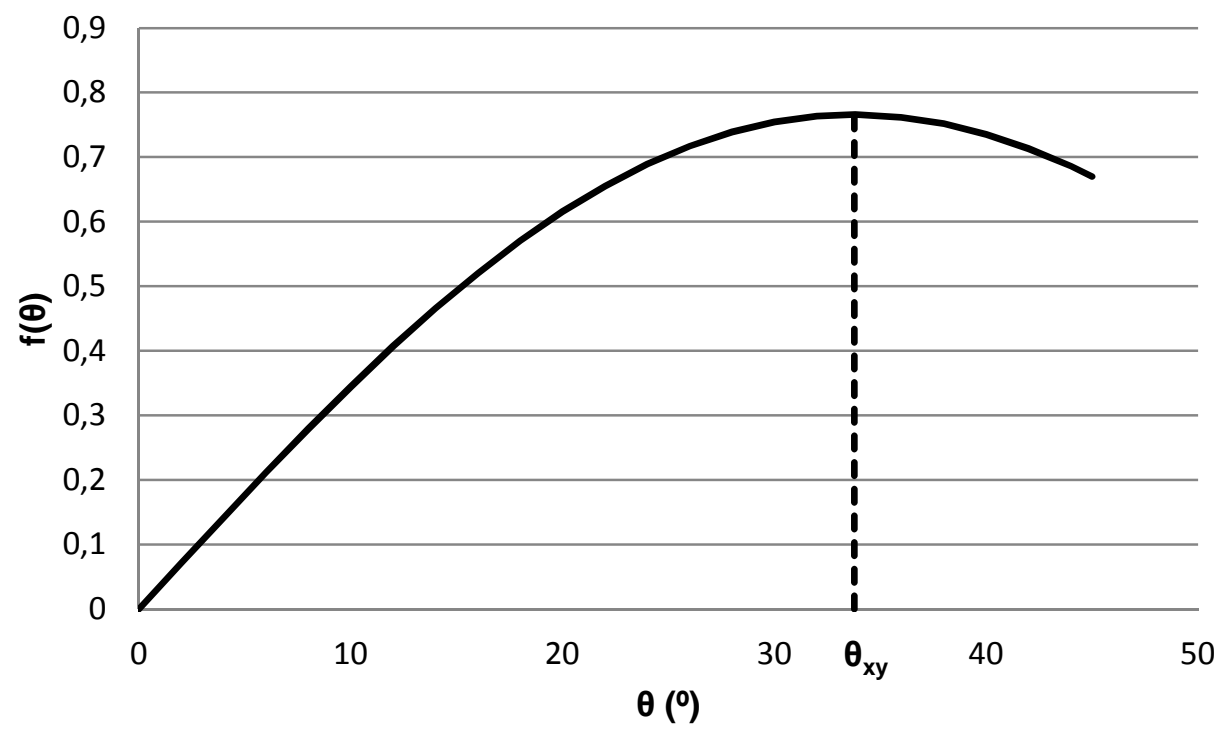

Figura 4.2 - Função $f(\theta)$

Iterativamente é possível encontrar o valor de $\theta_{x y}$. Assim, tem-que:

$\left|\theta_{x y}\right|=33,76^{\circ}$

Portanto:

$\left|n_{x y}\right| \leq \frac{f_{c d 1} \cdot h}{2} \cdot\left(\frac{\operatorname{sen}\left(2 \cdot\left|\theta_{x y}\right|\right)}{0,8+170 \cdot\left[\frac{2 \cdot \varepsilon_{y d}+2 \% 0 \cdot\left(1-\cos \left(2 \cdot\left|\theta_{x y}\right|\right)\right)}{\left(1+\cos \left(2 \cdot\left|\theta_{x y}\right|\right)\right)}\right]}\right)$

Para CA-50 e CA-60, como $\left|\theta^{*}\right|>\left|\theta_{2}\right|$, então a equação 4.10 é válida.

$$
\begin{aligned}
f_{c d 2} & =\frac{2 \cdot n_{x y}}{h \cdot \operatorname{sen}(2 \theta)} \\
n_{x y} & =\frac{f_{c d 2} \cdot h \cdot \operatorname{sen}(2 \theta)}{2}
\end{aligned}
$$

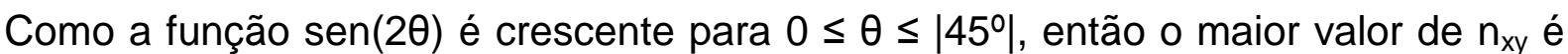
obtido para $\theta^{*}$, pois este será o maior valor de $\theta$ possível para esta situação. Assim, tem-se que: 
$\left|n_{x y}\right| \leq \frac{f_{c d 2} \cdot h \cdot \operatorname{sen}\left(2\left|\theta^{*}\right|\right)}{2}$

Portanto, se $n_{x y}$ respeitar a condição imposta por 4.11 ou 4.12, o problema sempre tem solução, ou seja, existe uma armadura que fará com que a tensão no concreto diminua até a sua resistência máxima. A Tabela 4.2 apresenta os valores de $\theta$ máximo para cada tipo de aço.

Tabela 4.2 - Valores máximos para $\theta$ para o caso III

\begin{tabular}{|c|c|c|}
\hline & $\varepsilon_{\text {yd }}(\% \circ)$ & $\theta_{\max }(\stackrel{\circ}{)})$ \\
\hline CA-25 & 1,04 & 33,76 \\
\hline CA-50 & 2,07 & 35,03 \\
\hline CA-60 & 2,48 & 33,74 \\
\hline
\end{tabular}

\subsection{Dimensionamento das armaduras}

Dada uma chapa que está submetida a esforços tais que respeitem as condições impostas no pelas equações 4.11 ou 4.12 , pretende-se calcular a quantidade de armadura necessária a ser posicionada na direção y de forma que a tensão de compressão no concreto seja igual à resistência máxima $f_{c 2 m a x}$. Este método de cálculo foi baseado naquele apresentado por Jazra (2008). Sendo assim, retomando as equações 4.6, 4.7, 4.8, 4.9 e 4.10, deseja-se encontrar o valor de $\theta$ que é solução do problema. Primeiramente, se $\mathrm{n}_{\mathrm{xy}}=0$, então $\theta=0$.

Se $\theta_{1}$ existe e $0<\theta \leq\left|\theta_{1}\right|$, então:

$$
\begin{aligned}
& f_{c d 1}=\frac{n_{x y}(\operatorname{tg} \theta+\operatorname{cotg} \theta)}{h} \\
& f_{c d 1} \cdot h=\frac{2 \cdot n_{x y}}{\operatorname{sen}(2 \theta)} \\
& \operatorname{sen}(2 \theta)=\frac{2 \cdot n_{x y}}{f_{c d 1} \cdot h} \\
& \theta=\frac{\operatorname{arcsen}\left(\frac{2 \cdot n_{x y}}{f_{c d 1} \cdot h}\right)}{2}
\end{aligned}
$$


Se $\theta_{1}$ existe e $\left|\theta_{1}\right|<\theta<\left|\theta_{2}\right|$ ou se $\theta_{1}$ não existe e $0^{\circ}<\theta<\left|\theta_{2}\right|$, então:

$\frac{f_{c d 1}}{0,8+170 \cdot\left[\frac{2 \cdot \varepsilon_{y d}+2 \% 0 \cdot(1-\cos 2 \theta)}{(1+\cos 2 \theta)}\right]}=\frac{n_{x y}(\operatorname{tg} \theta+\operatorname{cotg} \theta)}{h}$

$\frac{f_{c d 1} \cdot h}{0,8+170 \cdot\left[\frac{2 \cdot \varepsilon_{y d}+2 \% 0 \cdot(1-\cos 2 \theta)}{(1+\cos 2 \theta)}\right]}=\frac{2 \cdot n_{x y}}{\operatorname{sen}(2 \theta)}$

$\operatorname{sen}(2 \theta)=\frac{2 \cdot n_{x y}}{\frac{f_{c d 1} \cdot h}{0,8+170 \cdot\left[\frac{2 \cdot \varepsilon_{y d}+2 \% 0 \cdot(1-\cos 2 \theta)}{(1+\cos 2 \theta)}\right]}}$

$\theta=\frac{\operatorname{arcsen}\left(\frac{2 \cdot n_{x y}}{\frac{f_{c d 1} \cdot h}{0,8+170 \cdot\left[\frac{2 \cdot \varepsilon_{y d}+2 \% 0 \cdot(1-\cos 2 \theta)}{(1+\cos 2 \theta)}\right]}}\right)}{2}$

$E$, finalmente, se $\left|\theta_{2}\right| \leq \theta<\left|\theta^{*}\right|$, então:

$f_{c d 2}=\frac{n_{x y}(\operatorname{tg} \theta+\operatorname{cotg} \theta)}{h}$

$\theta=\frac{\operatorname{arcsen}\left(\frac{2 \cdot n_{x y}}{f_{c d 2} \cdot h}\right)}{2}$

Formado este quadro, para encontrar $\theta$ solução, deve-se seguir o roteiro abaixo.

1. Se $n_{x y}=0, \theta=0$.

2. Se $n_{x y} \neq 0$, utilizar método iterativo para achar $\theta$ através da equação 4.14.

3. Se convergir, para aço CA-25, pode ser encontradas duas soluções, porém só é válida aquela em que $\theta<\theta_{\max }$.

4. Se convergir, para aço CA-25, verificar se $\theta \leq \theta_{1}$. Como a equação 4.14 não é válida para este domínio, em caso positivo, deve-se encontrar $\theta$ através da equação 4.13.

5. Se convergir, para CA-50 ou CA-60, $\theta$ encontrado é solução.

6. Se não convergir, encontrar a solução através da equação 4.15. 
Como os valores de $\varepsilon_{x}=\varepsilon_{y d}, \varepsilon_{2}=\varepsilon_{c}^{\prime}=-2 \%$ e $\theta$, pode-se então obter o valor de $\varepsilon_{1} \mathrm{e}$ $\varepsilon_{y}$. Retomando a equação 3.18 e 3.14 , tem-se que:

$\varepsilon_{1}=\frac{2 \cdot \varepsilon_{x}-\varepsilon_{2} \cdot(1-\cos 2 \theta)}{(1+\cos 2 \theta)}$
$\varepsilon_{1}=\frac{2 \cdot \varepsilon_{y d}+2 \% 0 \cdot(1-\cos 2 \theta)}{(1+\cos 2 \theta)}$

$E$ :

$\varepsilon_{1}+\varepsilon_{2}=\varepsilon_{x}+\varepsilon_{y}$

$\varepsilon_{y}=\varepsilon_{1}+\varepsilon_{2}-\varepsilon_{x}$

Então:

$\varepsilon_{y}=\frac{2 \cdot \varepsilon_{y d}+2 \% 0 \cdot(1-\cos 2 \theta)}{(1+\cos 2 \theta)}-2 \% 0-\varepsilon_{y d}$

Calculam-se as forças nas armaduras através das equações 2.1 e 2.2.

$$
\begin{aligned}
& n_{s x}=n_{x}+n_{x y} \cdot \operatorname{tg} \theta \\
& n_{s y}=n_{y}+n_{x y} \cdot \operatorname{cotg} \theta
\end{aligned}
$$

As armaduras são dadas por:

$$
\begin{aligned}
& a_{s x}=\frac{n_{s x}}{\sigma_{x}}=\frac{n_{s x}}{E_{c s} \cdot \varepsilon_{x}}=\frac{n_{s x}}{E \cdot \varepsilon_{y d}}=\frac{n_{s x}}{f_{y d}} \\
& a_{s y}=\frac{n_{s y}}{\sigma_{y}}=\frac{n_{s y}}{E_{c s} \cdot \varepsilon_{y}}
\end{aligned}
$$




\subsection{Exemplos de dimensionamento}

Da mesma forma que no Capítulo 3, serão feitos alguns exemplos aplicando os conceitos expostos. Todos eles recaem no caso III de dimensionamento do CEB (1990). A resolução para o caso II é análoga. Em todos os exemplos deste estudo serão adotados os coeficientes de segurança recomendados pela NBR 6118. Todos os esforços solicitantes são de cálculo.

\subsubsection{Exemplo 4.1 - Tensão de compressão maior que $f_{c d 2}$}

Dado uma chapa com espessura de $12 \mathrm{~cm}, \mathrm{f}_{\mathrm{ck}}$ igual a $25 \mathrm{Mpa}, \mathrm{CA}-50$ e submetida a esforços por unidade de comprimento como mostrados na Figura 4.3.

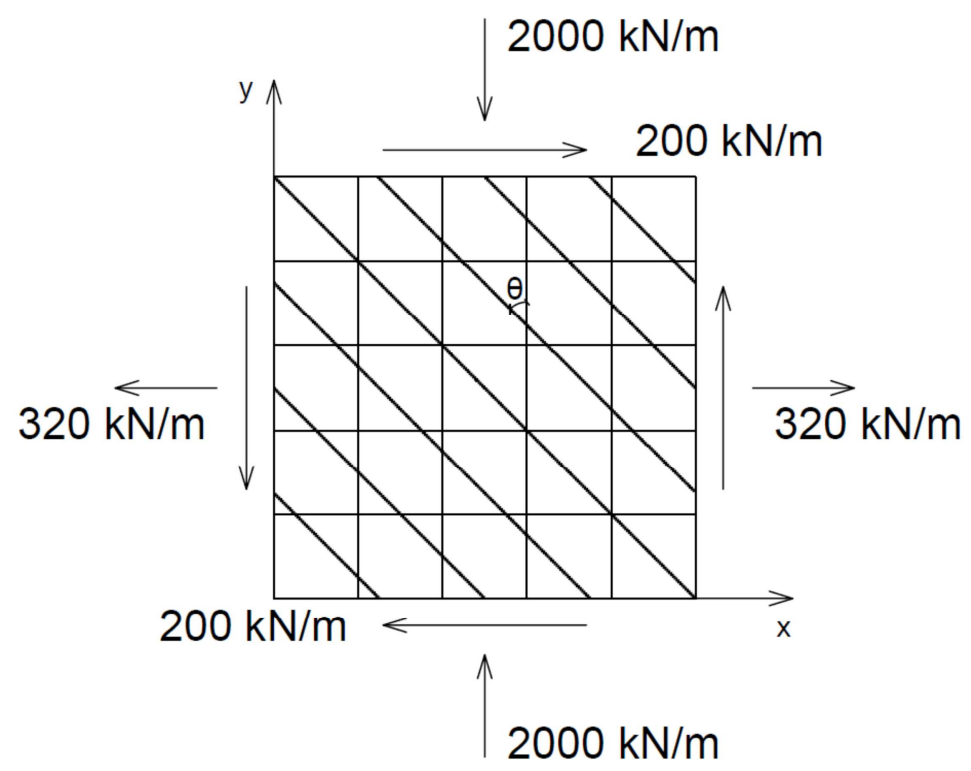

Figura 4.3 - Exemplo 4.1 - Tensão de compressão maior que fcd2

$$
\begin{aligned}
& h=12 \mathrm{~cm} \\
& f_{c k}=25 \mathrm{MPa} \\
& n_{x}=320 \mathrm{kN} / \mathrm{m} \\
& n_{y}=-2000 \mathrm{kN} / \mathrm{m} \\
& n_{x y}=200 \mathrm{kN} / \mathrm{m}
\end{aligned}
$$


Primeiramente, da mesma forma que no item 0 , verifica-se que caso de dimensionamento se aplica para esta chapa calculando os esforços $n_{s x}$ e $n_{s y}$ através das expressões 2.11 e 2.12 .

$n_{s x}=n_{x}+\left|n_{x y}\right|$

$n_{s y}=n_{y}+\left|n_{x y}\right|$

Então, tem-se que:

$n_{s x}=n_{x}+\left|n_{x y}\right|=320+|200|=500 \mathrm{kN} / \mathrm{m}$

$n_{s y}=n_{y}+\left|n_{x y}\right|=-2000+|200|=-1800 \mathrm{kN} / \mathrm{m}$

Como $\mathrm{n}_{\mathrm{sx}}>0$ e $\mathrm{n}_{\mathrm{sy}} \leq 0$, pode-se dispensar o uso de armaduras de tração na direção y, o que caracteriza o caso III de dimensionamento. Assim, primeiramente verifica-se o concreto:

$$
\begin{aligned}
& \theta=\operatorname{arctg}\left(-\frac{n_{x y}}{n_{y}}\right)=\operatorname{arctg}\left(-\frac{200}{-2000}\right)=5,71^{\circ} \\
& n_{c}=n_{x y \cdot} \cdot(\operatorname{tg} \theta+\operatorname{cotg} \theta) \\
& n_{c}=200 \cdot\left(\operatorname{tg} 5,71^{\circ}+\operatorname{cotg} 5,71^{\circ}\right) \\
& n_{c}=2020 \mathrm{kN} / \mathrm{m} \\
& \sigma_{c}=\frac{n_{c}}{h}=\frac{2020}{0,12}=16833,33 \frac{\mathrm{kN}}{\mathrm{m}^{2}}=16,83 \mathrm{MPa} \\
& f_{c d 1}=0,85 \cdot\left(1-\frac{f_{c k}}{250}\right) \cdot f_{c d}=0,85 \cdot\left(1-\frac{25}{250}\right) \cdot \frac{25}{1,4}=13,66 \mathrm{MPa} \\
& f_{c d 2}=0,60 \cdot\left(1-\frac{f_{c k}}{250}\right) \cdot f_{c d}=0,60 \cdot\left(1-\frac{f_{c k}}{250}\right) \cdot \frac{25}{1,4}=9,64 \mathrm{MPa}
\end{aligned}
$$

Como neste caso $\sigma_{c}>f_{c d 1}$, a tensão de compressão no concreto está acima do limite máximo de resistência. Então, verifica-se a possibilidade de adotar armaduras de compressão na direção y para auxiliar o equilíbrio do esforço de compressão no concreto.

Primeiramente, como foi adotado aço CA-50, deve-se avaliar o critério exposto pela equação 4.12 . 
$\left|n_{x y}\right| \leq \frac{f_{c d 2} \cdot h \cdot \operatorname{sen}\left(2\left|\theta^{*}\right|\right)}{2}$

Calcula-se $\theta^{*}$ através da equação 4.5 .

$\theta^{*}=\frac{\arccos \left(\frac{\varepsilon_{y d}}{\varepsilon_{y d}+2.2}\right)}{2}$

$\theta^{*}=\frac{\arccos \left(\frac{2,07}{2,07+2.2}\right)}{2}=\frac{\arccos \left(\frac{2,07}{6,07}\right)}{2}=35,03^{\circ}$

Agora, encontra-se o valor de $\mathrm{n}_{\mathrm{xy}}$ limite:

$\left|n_{x y}\right| \leq \frac{9642,9.0,12 \cdot|\operatorname{sen}(2.35,03)|}{2}=543,7 \mathrm{kN} / \mathrm{m}$

Portanto, como $\mathrm{n}_{\mathrm{xy}}$ do problema é menor que $\mathrm{n}_{\mathrm{xy}}$ limite, é possível calcular armaduras de compressão para este caso. Assim, através da equação 4.14, tem-se que:

$\theta=\frac{\operatorname{arcsen}\left(\frac{2 \cdot n_{x y}}{\frac{f_{c d 1} \cdot h}{0,8+170 \cdot\left[\frac{2 \cdot \varepsilon_{y d}+2 \% 0 \cdot(1-\cos 2 \theta)}{(1+\cos 2 \theta)}\right]}}\right)}{2}$

$\theta=\frac{\operatorname{arcsen}\left(\frac{2.200}{\frac{13660,7 \cdot 0,12}{0,8+170 \cdot\left[\frac{2.2,07 \% 0+2 \% \cdot(1-\cos 2 \theta)}{(1+\cos 2 \theta)}\right]}}\right)}{2}$

Aplicando o roteiro apresentado no item 4.3, iterativamente verifica-se se é possível obter o valor de $\theta$. Pode se ver pela Tabela 4.3 que neste caso o valor de $\theta$ converge. 
Tabela 4.3 - Cálculo iterativo $\theta$ para o exemplo 4.1

\begin{tabular}{|c|c|c|c|c|c|}
\hline $\mathrm{i}$ & $\theta_{\mathrm{i}}(\stackrel{\circ}{)})$ & $\varepsilon_{1}(\% \mathrm{o})$ & $\mathrm{f}_{\mathrm{c} 2 \max }(\mathrm{MPa})$ & $\sigma_{\mathrm{c}}(\mathrm{MPa})$ & $\theta_{\mathrm{f}}(\stackrel{\circ}{)})$ \\
\hline 1 & 1,000 & 2,072 & 11,856 & 95,512 & 8,164 \\
\hline 2 & 8,164 & 2,154 & 11,714 & 11,856 & 8,266 \\
\hline 3 & 8,266 & 2,156 & 11,710 & 11,714 & 8,269 \\
\hline 4 & 8,269 & 2,156 & 11,710 & 11,710 & 8,269 \\
\hline 5 & 8,269 & 2,156 & 11,710 & 11,710 & 8,269 \\
\hline 6 & 8,269 & 2,156 & 11,710 & 11,710 & 8,269 \\
\hline
\end{tabular}

Como neste exemplo está sendo admitido que o aço é CA-50, não há necessidade de se verificar se $\theta \leq \theta_{1}$, pois $\theta_{1}$ não existe.

A Figura 4.4 apresenta os gráficos de $\mathrm{f}_{\mathrm{c} 2 \mathrm{max}}$ e de $\sigma_{\mathrm{c}}$, em outras palavras, da tensão resistente e da tensão solicitante na chapa. Pode-se observar que como as curvas se cruzam, existe solução para o problema e ela foi calculada pela Tabela 4.3. Também se pode ver que quando $\theta=0^{\circ}, f_{c 2 m a x}$ assume seu valor máximo, porém não é igual a $f_{c d 1}$, pois está sendo considerado aço CA-50, conforme mostrado no item 4.3.

Como, por hipótese $\varepsilon_{x}=\varepsilon_{y d}$ e para CA50 $\varepsilon_{y d}=2,07 \%$, quando $\theta=0^{\circ}$ as direções $x$ e y são direções principais de deformação e, portanto, $\varepsilon_{x}=\varepsilon_{y d}=\varepsilon_{1}$. Assim, o valor máximo da resistência é:

$f_{c 2 \max }=\frac{f_{c d 1}}{0,8+170 . \varepsilon_{1}}$

$f_{c 2 \max }=\frac{13,66}{0,8+170.2,07 \% 0}=11,86 \mathrm{MPa}$ 


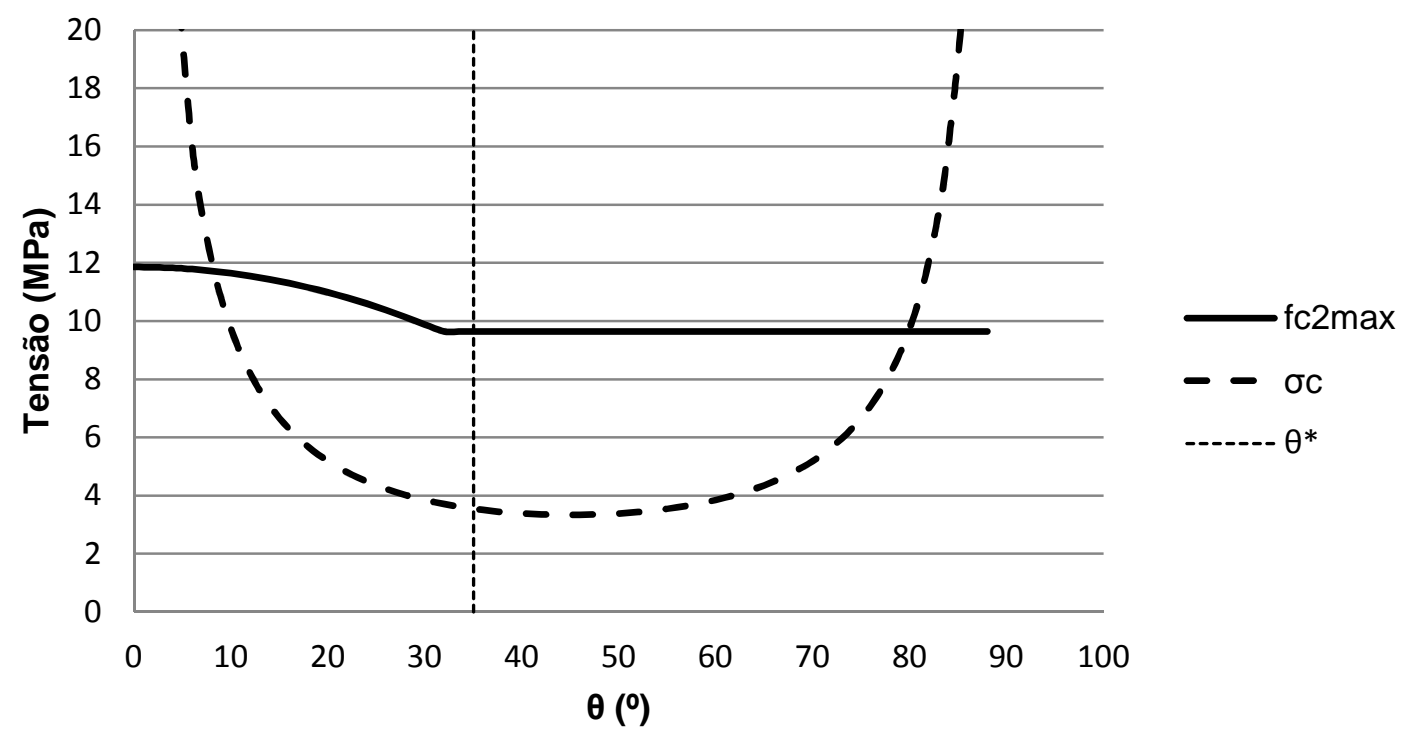

Figura 4.4 - Gráfico Tensão x $\theta$ para exemplo 4.1

Prosseguindo, agora é possível calcular $\varepsilon_{y}$.

$$
\begin{aligned}
& \varepsilon_{y}=\frac{2 \cdot \varepsilon_{y d}+2 \% 0 \cdot(1-\cos 2 \theta)}{(1+\cos 2 \theta)}-2 \% 0-\varepsilon_{y d} \\
& \varepsilon_{y}=\frac{2.2,07 \% 0+2 \% 0 \cdot\left(1-\cos \left(2.8,27^{\circ}\right)\right)}{\left(1+\cos \left(2.8,27^{\circ}\right)\right)}-2 \% 0-2,07 \% 0=-1,91 \% 0
\end{aligned}
$$

Então, calculam-se os esforços nas armaduras.

$$
\begin{aligned}
& n_{s x}=n_{x}+n_{x y} \cdot \operatorname{tg} \theta \\
& n_{s x}=320+200 \cdot \operatorname{tg} 8,27^{\circ}=329,07 \mathrm{kN} / \mathrm{m} \\
& n_{s y}=n_{y}+n_{x y} \cdot \operatorname{cotg} \theta \\
& n_{s y}=-2000+200 \cdot \operatorname{cotg} 8,27^{\circ}=-623,78 \mathrm{kN} / \mathrm{m}
\end{aligned}
$$

Finalmente, as armaduras são dadas por:

$$
\begin{aligned}
& a_{s x}=\frac{n_{s x}}{f_{y d}}=\frac{329,07}{43,49}=7,57 \mathrm{~cm}^{2} \\
& a_{s y}=\frac{n_{s y}}{\sigma_{y}}=\frac{n_{s y}}{E \cdot \varepsilon_{y}}=\frac{-623,78}{21000 \cdot(-1,91 \% 0)}=15,52 \mathrm{~cm}^{2}
\end{aligned}
$$




\subsubsection{Exemplo 4.2 - Tensão de compressão igual a $f_{c d 2}$}

Utilizando a mesma chapa do exemplo 4.1, mas desta vez com o esforço de cisalhamento maior, conforme mostrado Figura 4.5.

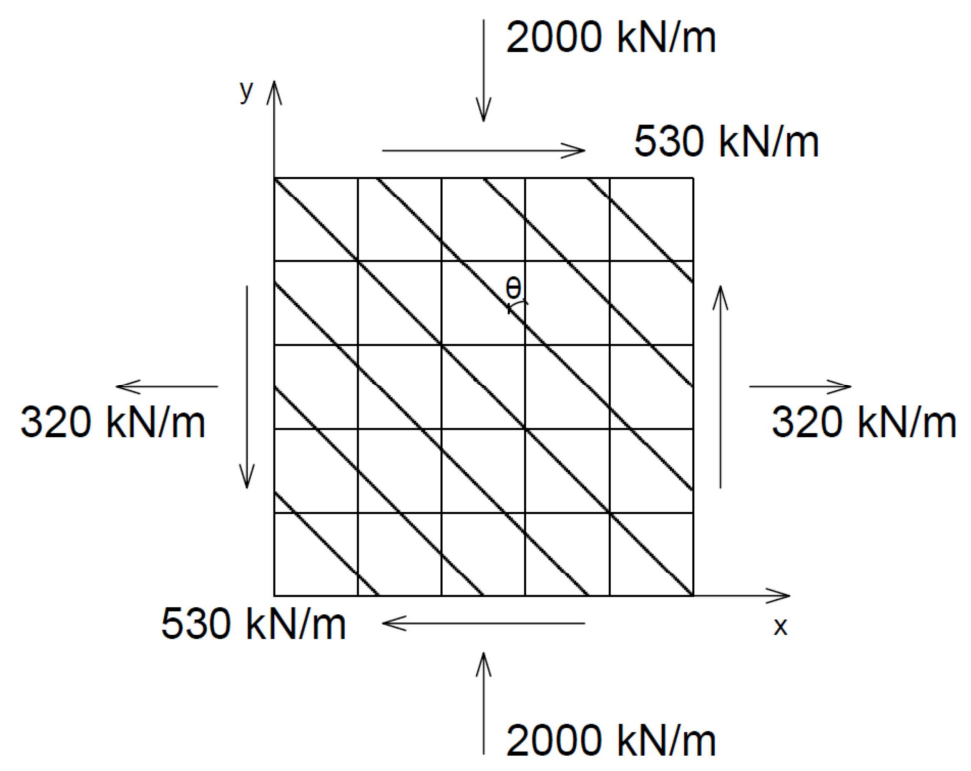

Figura 4.5 - Exemplo 4.2 - Tensão de compressão igual a fcd2

$h=12 \mathrm{~cm}$

$f_{c k}=25 \mathrm{MPa}$

$n_{x}=320 \mathrm{kN} / \mathrm{m}$

$n_{y}=-2000 \mathrm{kN} / \mathrm{m}$

$n_{x y}=530 \mathrm{kN} / \mathrm{m}$

Como no exemplo anterior, esta chapa recai no caso III de dimensionamento e também a tensão de compressão no concreto é maior que $\mathrm{f}_{\mathrm{cd}}$.

$\sigma_{c}=\frac{n_{c}}{h}=17,84 M P a>f_{c d 1}$

Dado isto, verifica-se se é possível adotar armaduras de compressão na direção y. Como no exemplo anterior, deve-se avaliar o critério exposto pela equação 4.12. 
$\left|n_{x y}\right| \leq \frac{f_{c d 2} \cdot h \cdot \operatorname{sen}\left(2\left|\theta^{*}\right|\right)}{2}$

Os cálculos são idênticos aos do exemplo 4.1 e resultam em:

$\theta^{*}=35,03^{\circ}$

$\left|n_{x y}\right| \leq 543,7 \mathrm{kN} / \mathrm{m}$

Portanto, como $\mathrm{n}_{\mathrm{xy}}$ do problema é menor que $\mathrm{n}_{\mathrm{xy}}$ limite, é possível calcular armaduras de compressão para este caso. Assim, seguindo o roteiro do item 4.3, através da equação 4.14 , tem-se que:

$\theta=\frac{\operatorname{arcsen}\left(\frac{2 \cdot n_{x y}}{\frac{f_{c d 1} \cdot h}{0,8+170 \cdot\left[\frac{2 \cdot \varepsilon_{y d}+2 \% \cdot(1-\cos 2 \theta)}{(1+\cos 2 \theta)}\right]}}\right)}{2}$

$\theta=\frac{\operatorname{arcsen}\left(\frac{2.200}{\frac{13660,7 \cdot 0,12}{0,8+170 \cdot\left[\frac{2.2,07 \% 0+2 \% 0 \cdot(1-\cos 2 \theta)}{(1+\cos 2 \theta)}\right]}}\right)}{2}$

Da mesma forma que no exemplo 4.1, iterativamente, deve-se analisar se $\theta$ converge. Através da Tabela 4.4, verifica-se que para esse caso $\theta$ não converge.

Tabela 4.4 - Cálculo iterativo $\theta$ para o exemplo 4.2

\begin{tabular}{|c|c|c|c|c|c|}
\hline $\mathrm{i}$ & $\theta_{\mathrm{i}}(\stackrel{\circ}{)})$ & $\varepsilon_{1}(\%)$ & $\mathrm{f}_{\mathrm{c} 2 \max }(\mathrm{MPa})$ & $\sigma_{\mathrm{c}}(\mathrm{MPa})$ & $\theta_{\mathrm{f}}(\stackrel{\circ}{)})$ \\
\hline 1 & 1,000 & 2,072 & 11,856 & 257,883 & 24,692 \\
\hline 2 & 24,692 & 2,931 & 10,522 & 11,856 & 29,397 \\
\hline 3 & 29,397 & 3,362 & 9,960 & 10,522 & 32,321 \\
\hline 4 & 32,321 & 3,700 & 9,560 & 9,960 & 35,147 \\
\hline 5 & 35,147 & 4,088 & 9,138 & 9,560 & 40,016 \\
\hline 6 & 40,016 & 4,940 & 8,331 & 9,138 & \#NÚM! \\
\hline
\end{tabular}


Como $\theta$ não convergiu, deve-se utilizar então a equação 4.15 .

$\theta=\frac{\operatorname{arcsen}\left(\frac{2 \cdot n_{x y}}{f_{c d 2} \cdot h}\right)}{2}$

$\theta=\frac{\operatorname{arcsen}\left(\frac{2.530}{9642,86.0,12}\right)}{2}=33,178^{\circ}$

Para este exemplo, o gráfico tensão $\times \theta$ apresentado na Figura 4.6 mostra que as curvas se cruzam na região em que a tensão resistente vale $\mathrm{f}_{\mathrm{cd} 2}$, conforme já previsto pelo equacionamento.

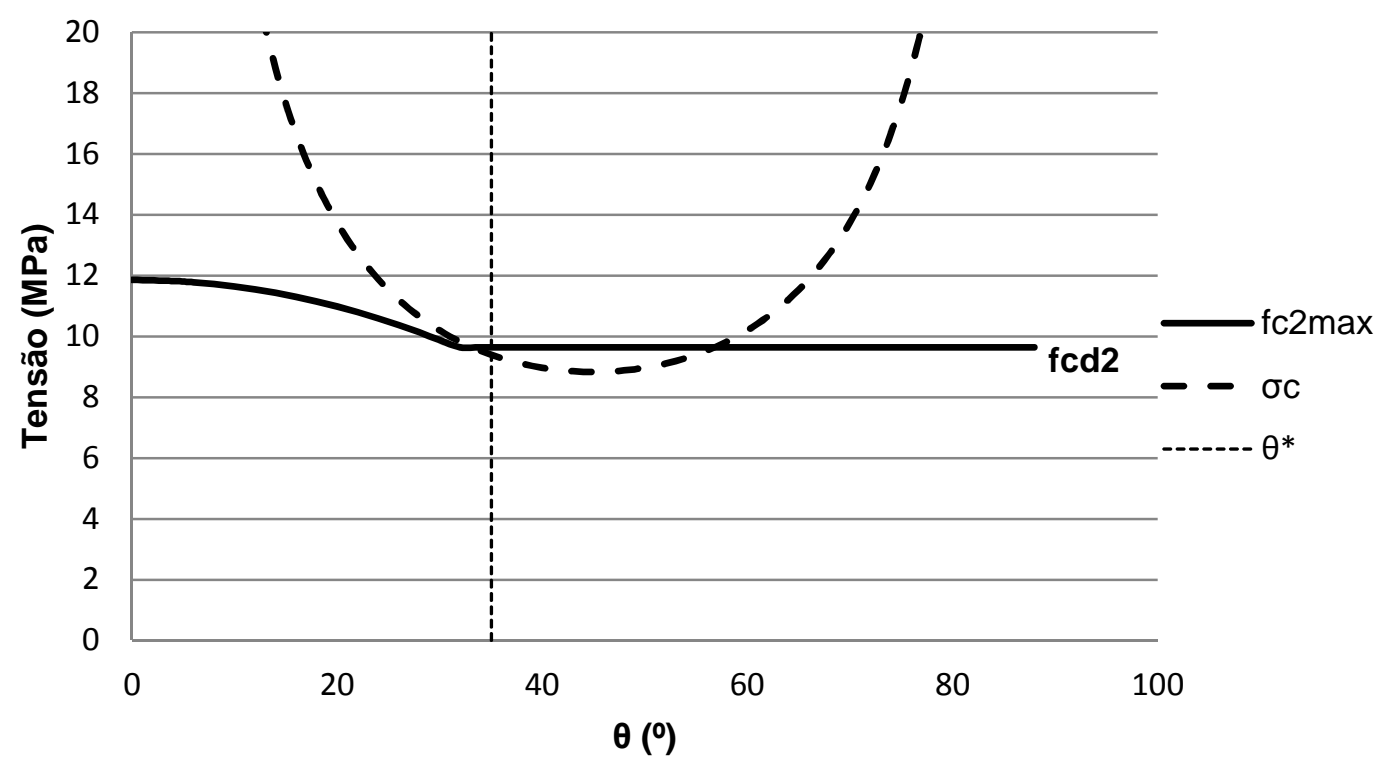

Figura 4.6 - Gráfico Tensão x $\theta$ para exemplo 4.2

Então, agora calcula-se $\varepsilon_{y}$ :

$$
\begin{aligned}
& \varepsilon_{y}=\frac{2 \cdot \varepsilon_{y d}+2 \% 0 \cdot(1-\cos 2 \theta)}{(1+\cos 2 \theta)}-2 \% 0-\varepsilon_{y d} \\
& \varepsilon_{y}=\frac{2.2,07 \% 0+2 \% 0 \cdot\left(1-\cos \left(2 \cdot 33,178^{\circ}\right)\right)}{\left(1+\cos \left(2.33,178^{\circ}\right)\right)}-2 \% 0-2,07 \% 0=-0,26 \% 0
\end{aligned}
$$


Obtêm-se os esforços nas armaduras.

$n_{s x}=n_{x}+n_{x y} \cdot \operatorname{tg} \theta$

$n_{s x}=300+530 \cdot \operatorname{tg} 33,178^{\circ}=646,53 \mathrm{kN} / \mathrm{m}$

$n_{s y}=n_{y}+n_{x y} \cdot \operatorname{cotg} \theta$

$n_{s y}=-2000+530 \cdot \operatorname{cotg} 33,178^{\circ}=-1189,38 \mathrm{kN} / \mathrm{m}$

Finalmente, as armaduras são dadas por:

$$
\begin{aligned}
& a_{s x}=\frac{n_{s x}}{f_{y d}}=\frac{646,53}{43,49}=14,87 \mathrm{~cm}^{2} \\
& a_{s y}=\frac{n_{s y}}{\sigma_{y}}=\frac{n_{s y}}{E \cdot \varepsilon_{y}}=\frac{-1189,38}{21000 \cdot(-0,26 \% 0)}=217,72 \mathrm{~cm}^{2}
\end{aligned}
$$

\subsubsection{Exemplo 4.3 - Tensão de cisalhamento maior que o limite}

Neste exemplo, o esforço de cisalhamento será maior que o prescrito pela equação 4.12 a fim de mostrar os problemas que acontecem nesta situação. Os esforços adotados estão apresentados na Figura 4.7. 


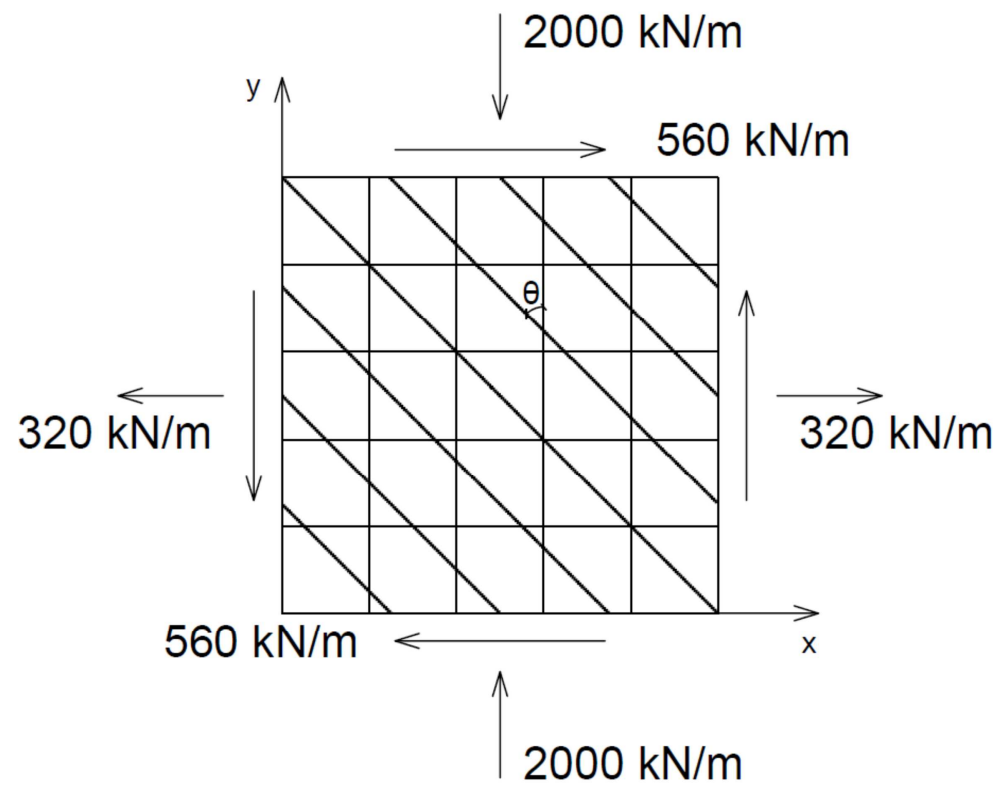

Figura 4.7 - Exemplo 4.3 - Tensão de cisalhamento maior que o limite

$h=12 \mathrm{~cm}$

$f_{c k}=25 \mathrm{MPa}$

$n_{x}=320 \mathrm{kN} / \mathrm{m}$

$n_{y}=-2000 \mathrm{kN} / \mathrm{m}$

$n_{x y}=560 \mathrm{kN} / \mathrm{m}$

Como no exemplo anterior, esta chapa recai no caso III de dimensionamento e também a tensão de compressão no concreto é maior que $\mathrm{f}_{\mathrm{cd} 1}$.

$\sigma_{c}=\frac{n_{c}}{h}=17,97 M P a>f_{c d 1}$

Dado isto, verifica-se se é possível adotar armaduras de compressão na direção y.

$\left|n_{x y}\right| \leq \frac{f_{c d 2} \cdot h \cdot\left|\operatorname{sen} 2 \theta^{*}\right|}{2}$

Os cálculos são idênticos aos do exemplo 4.1 e resultam em:

$\theta^{*}=35,03^{\circ}$

$\left|n_{x y}\right| \leq 543,7 \mathrm{kN} / \mathrm{m}$ 
Conforme exposto, este exemplo não respeita o critério apontado pela equação 4.12. Mesmo assim, se continuará com o dimensionamento.

Como se pode ver pela Tabela 4.1 e pelo cálculo de $\theta^{*}$, como foi adotado aço CA50, $\theta_{2}<\theta^{*}$. Da mesma forma, como a tensão de cisalhamento está acima do limite, $\theta$ só pode assumir valores maiores que $\theta^{*}$. Sendo assim, $\theta>\theta_{2}$ e, portanto, a equação 4.15 é válida.

$\theta=\frac{\operatorname{arcsen}\left(\frac{2 \cdot n_{x y}}{f_{c d 2} \cdot h}\right)}{2}$

$\theta=\frac{\operatorname{arcsen}\left(\frac{2.560}{9642,86.0,12}\right)}{2}=37,72^{\circ}$

Para este exemplo, o gráfico tensão $\times \theta$ apresentado pela Figura 4.8 mostra que as curvas se cruzam a direita da linha de $\theta^{*}$.

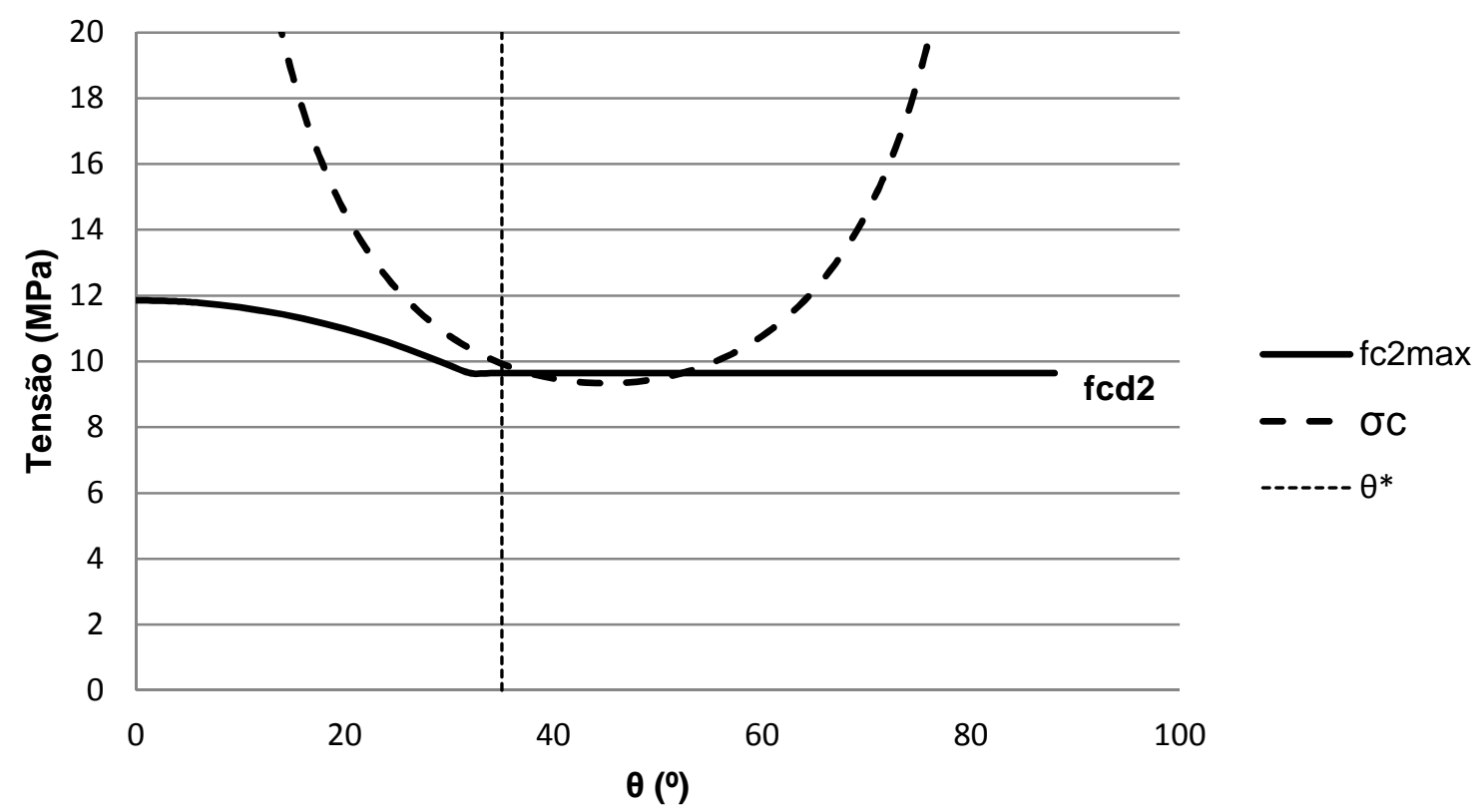

Figura 4.8 - Gráfico Tensão x $\theta$ para exemplo 4.3

Então, calcula-se $\varepsilon_{y}$ :

$\varepsilon_{y}=\frac{2 \cdot \varepsilon_{y d}+2 \% 0 \cdot(1-\cos 2 \theta)}{(1+\cos 2 \theta)}-2 \% 0-\varepsilon_{y d}$ 
$\varepsilon_{y}=\frac{2.2,07 \% 0+2 \% 0 \cdot\left(1-\cos \left(2.37,72^{\circ}\right)\right)}{\left(1+\cos \left(2 \cdot 37,72^{\circ}\right)\right)}-2 \% 0-2,07 \% 0=0,44 \% 0$

Obtêm-se as forças nas armaduras.

$n_{s x}=n_{x}+n_{x y} \cdot \operatorname{tg} \theta$

$n_{s x}=300+560 \cdot \operatorname{tg} 37,72^{\circ}=733,16 \mathrm{kN} / \mathrm{m}$

$n_{s y}=n_{y}+n_{x y} \cdot \operatorname{cotg} \theta$

$n_{s y}=-2000+560 \cdot \operatorname{cotg} 37,72^{\circ}=-1276,02 \mathrm{kN} / \mathrm{m}$

Finalmente, as armaduras são dadas por:

$$
\begin{aligned}
& a_{s x}=\frac{n_{s x}}{f_{y d}}=\frac{733,16}{43,49}=16,86 \mathrm{~cm}^{2} \\
& a_{s y}=\frac{n_{s y}}{\sigma_{y}}=\frac{n_{s y}}{E \cdot \varepsilon_{y}}=\frac{-1276,02}{21000 \cdot(0,44 \%)}=-139,66 \mathrm{~cm}^{2}
\end{aligned}
$$

Pode-se ver que como a deformação $\varepsilon_{y}$ resultou em um valor positivo, a armadura $a_{\text {sy }}$, por consequência, assume valor negativo o que se revela uma incongruência.

\subsubsection{Exemplo 4.4 - Problema com solução inexistente}

Neste exemplo, o esforço de cisalhamento será ainda maior que o do exemplo 4.4 sendo que desta vez não existirá um valor de $\theta$ que solucione o problema. Assim, os esforços adotados estão apresentados na Figura 4.9. 


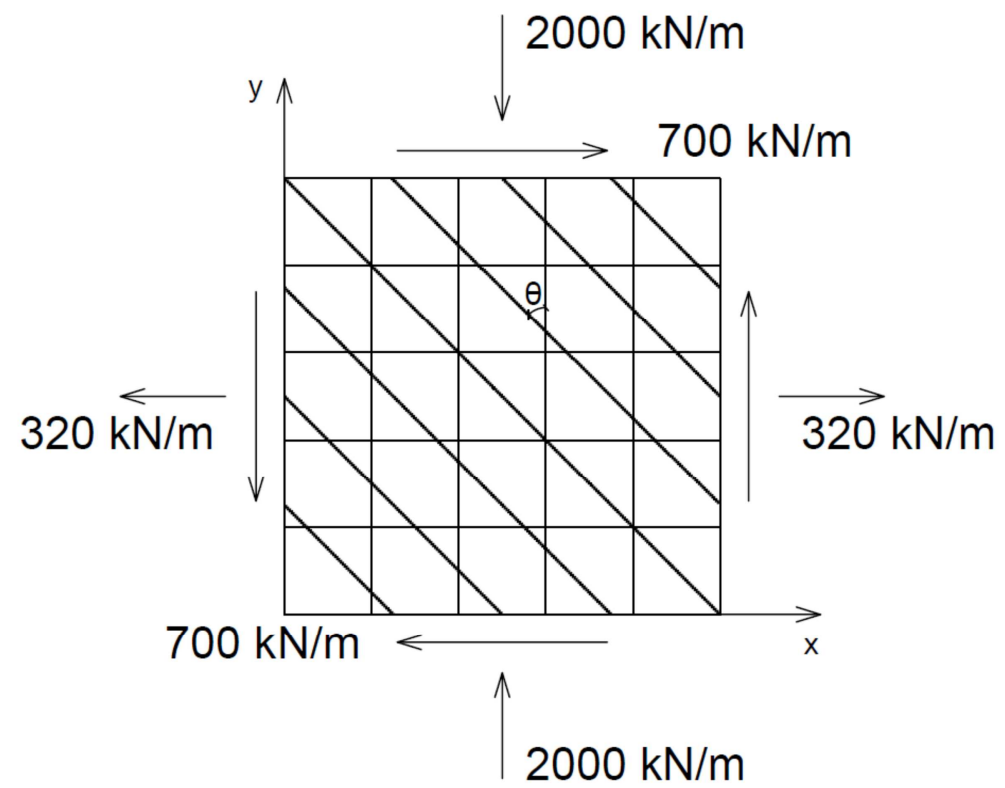

Figura 4.9 - Exemplo 4.4 - Problema com solução inexistente

$h=12 \mathrm{~cm}$

$f_{c k}=25 \mathrm{MPa}$

$n_{x}=320 \mathrm{kN} / \mathrm{m}$

$n_{y}=-2000 \mathrm{kN} / \mathrm{m}$

$n_{x y}=700 \mathrm{kN} / \mathrm{m}$

Como no exemplo anterior, esta chapa recai no caso III de dimensionamento e também a tensão de compressão no concreto é maior que $\mathrm{f}_{\mathrm{cd} 1}$.

$\sigma_{c}=\frac{n_{c}}{h}=18,71 M P a>f_{c d 1}$

Dado isto, verifica-se se é possível adotar armaduras de compressão na direção y.

$\left|n_{x y}\right| \leq \frac{f_{c d 2} \cdot h \cdot\left|\operatorname{sen} 2 \theta^{*}\right|}{2}$

Os cálculos são idênticos aos do exemplo 4.1 e resultam em:

$\theta^{*}=35,03^{\circ}$

$\left|n_{x y}\right| \leq 543,7 \mathrm{kN} / \mathrm{m}$ 
Este exemplo também não respeita o critério apontado pela equação 4.12. Mesmo assim se tentará continuar com o dimensionamento. Então, pelo mesmo motivo apontado no exemplo 4.3, a equação 4.15 é válida.

$\theta=\frac{\operatorname{arcsen}\left(\frac{2 \cdot n_{x y}}{f_{c d 2} \cdot h}\right)}{2}$

$\theta=\frac{\operatorname{arcsen}\left(\frac{2.700}{9642,86.0,12}\right)}{2}=\frac{\operatorname{arcsen}(1,21)}{2} \therefore \theta$ N $\tilde{A} O$ EXISTE

Para este exemplo, o gráfico tensão $\times \theta$ apresentado pela Figura 4.10, mostra que as curvas não se cruzam e, portanto, é impossível dimensionar esse caso.

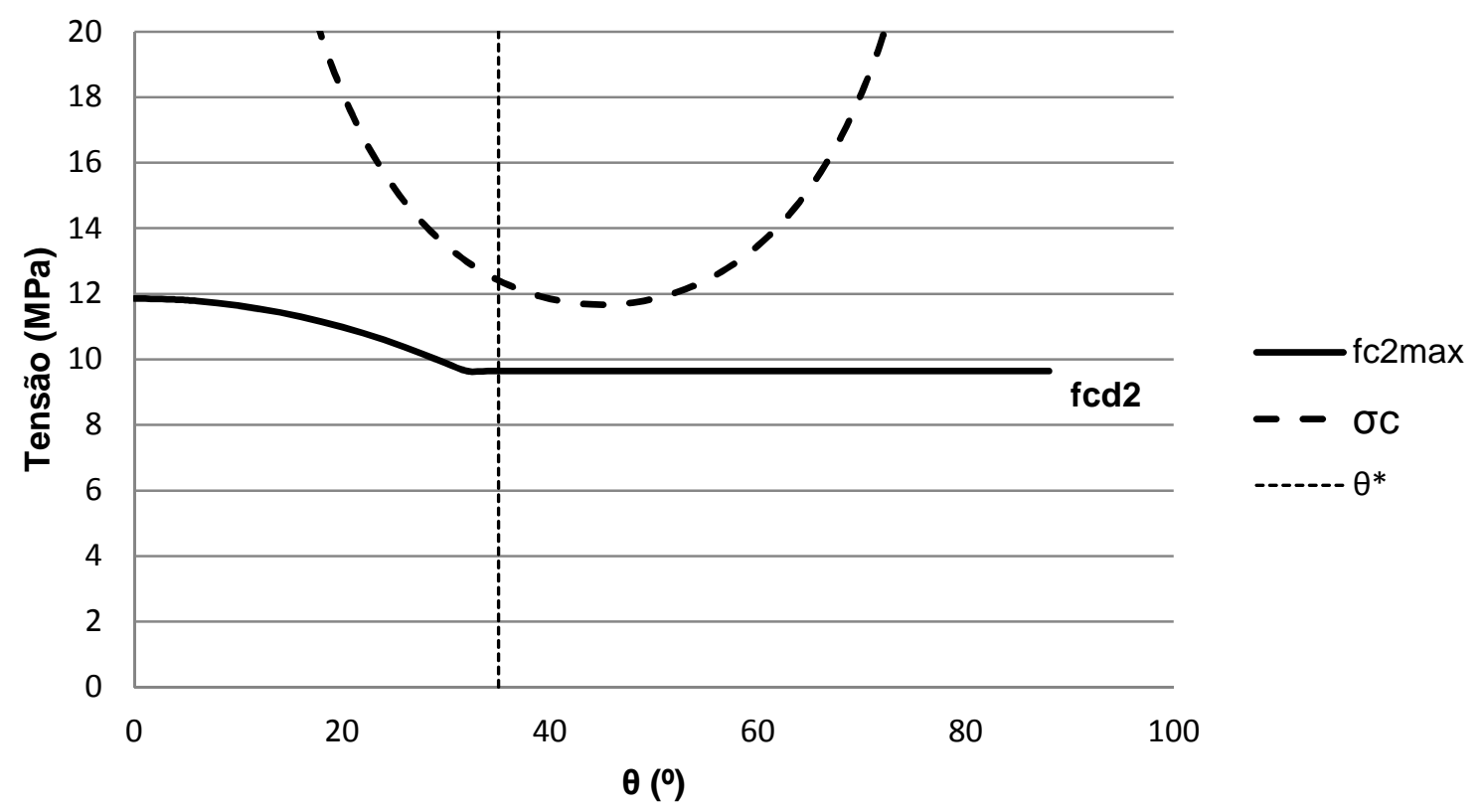

Figura 4.10 - Gráfico Tensão x $\theta$ para exemplo 4.4

\subsection{Dimensionamento das armaduras para o caso II}

Neste item será apresentada a formulação para o dimensionamento das armaduras de compressão para o caso II. Conforme já exposto, a demonstração das fórmulas é a mesma para o caso III e aqui serão apresentadas apenas as equações finais. 
Sendo assim, para o caso II também se deve respeitar os limite de dimensionamento apresentado no item 4.3, porém o domínio das funções de $\theta$ é $\left|45^{\circ}\right| \geq \theta \geq\left|90^{\circ}\right|$. Assim, como agora $\varepsilon_{y}=\varepsilon_{y d}$, adaptando a expressão $3.5, f_{c 2 m a x}$ é representado pela equação 4.19 e seu gráfico é dado pela

$$
f_{c 2 \max }=\frac{f_{c d 1}}{0,8+170 \cdot\left[\frac{2 \cdot \varepsilon_{y d}+2 \% 0 \cdot(1+\cos 2 \theta)}{(1-\cos 2 \theta)}\right]}
$$

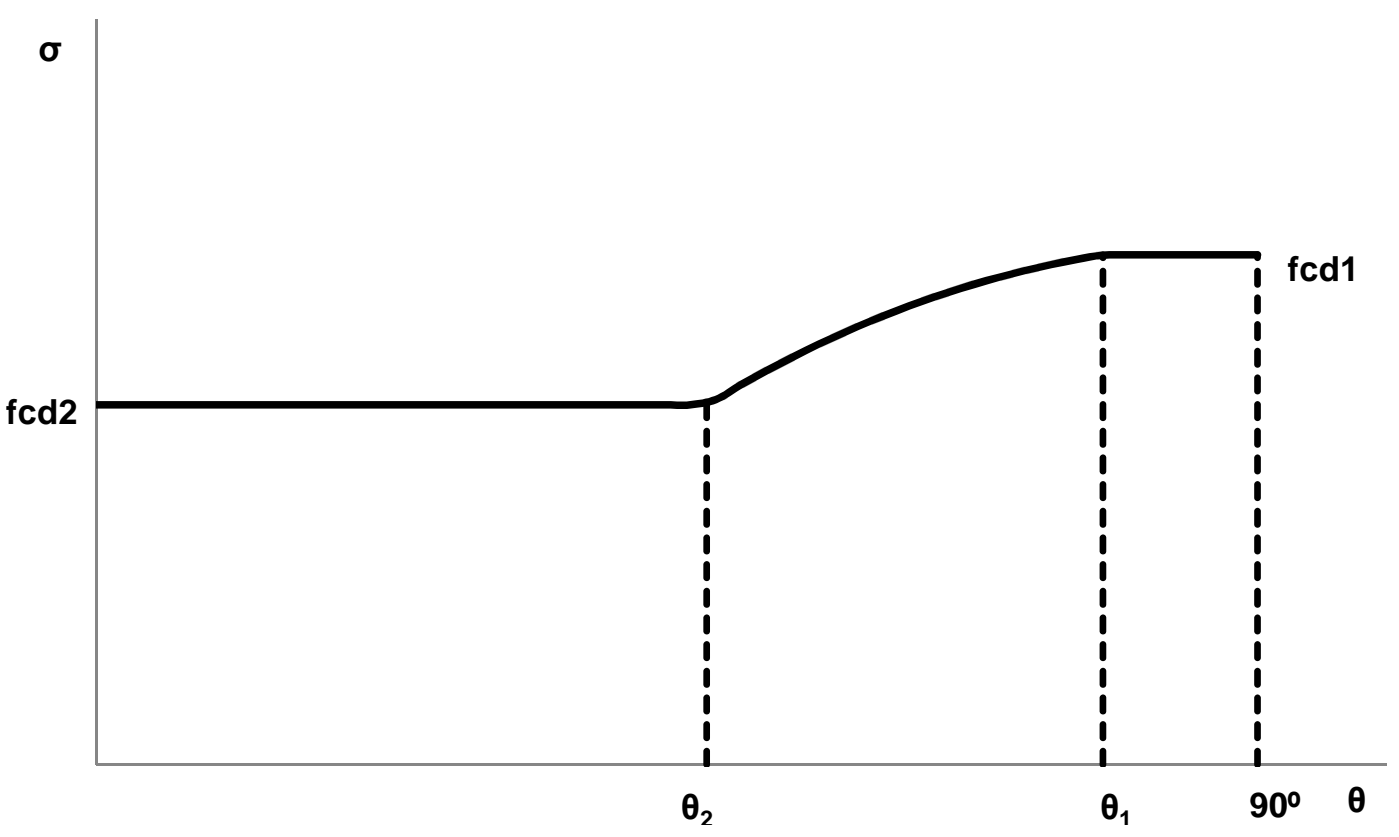

Figura 4.11 - Resistência à compressão do concreto em função de $\theta$ para o caso II

Assim, para o caso II, os limites de $\theta$ assumem os valores apresente na Tabela 4.5 e na Tabela 4.6.

Tabela 4.5 - Valores de $\theta_{1}, \theta_{2}$ e $\theta^{*}$ para os aços prescritos pela NBR 6118 para o caso II

\begin{tabular}{|c|c|c|c|c|}
\hline & $\varepsilon_{\text {yd }}(\% \circ)$ & $\left|\theta_{1}\right|\left(^{\circ}\right)$ & $\left|\theta_{2}\right|\left(^{(}\right)$ & $\left|\theta^{*}\right|\left(^{\circ}\right)$ \\
\hline CA-25 & 1,04 & 77,83 & 47,26 & 50,93 \\
\hline CA-50 & 2,07 & NÃO EXISTE & 58,26 & 54,97 \\
\hline CA-60 & 2,48 & NÃO EXISTE & 63,21 & 56,26 \\
\hline
\end{tabular}


Tabela 4.6 - Valores máximos para $\theta$ para o caso II

\begin{tabular}{|c|c|c|}
\hline & $\varepsilon_{\text {yd }}(\% \circ)$ & $\theta_{\max }(\stackrel{\circ}{)})$ \\
\hline CA-25 & 1,04 & 56.24 \\
\hline CA-50 & 2,07 & 54,97 \\
\hline CA-60 & 2,48 & 56,26 \\
\hline
\end{tabular}

Para CA-25, como $\left|\theta^{*}\right|<\left|\theta_{2}\right|$, a equação 4.12 nunca será válida. Então, retomando a equação 4.11, deseja-se saber qual o máximo valor que $n_{x y}$ pode assumir.

Portanto para CA-25, tem-se que:

$\left|n_{x y}\right| \leq \frac{f_{c d 1} \cdot h}{2} \cdot\left(\frac{\operatorname{sen}\left(2 \cdot\left|\theta_{\max }\right|\right)}{0,8+170 \cdot\left[\frac{2 \cdot \varepsilon_{y d}+2 \% 0 \cdot\left(1+\cos \left(2 \cdot\left|\theta_{\max }\right|\right)\right)}{\left(1-\cos \left(2 \cdot\left|\theta_{\max }\right|\right)\right)}\right]}\right)$

Para CA-50 e CA-60, tem-se que:

$\left|n_{x y}\right| \leq \frac{f_{c d 2} \cdot h \cdot \operatorname{sen}\left(2\left|\theta_{\max }\right|\right)}{2}$

Se caso o esforço de cisalhamento para o qual a chapa está submetida respeitar a condição 4.20 ou 4.21, então se deve encontrar o valor de $\theta$ utilizando o mesmo roteiro que aquele para o caso III, porém utilizando a formulação encontrada neste item. Primeiramente, se $\mathrm{n}_{\mathrm{xy}}=0$, então $\theta=90^{\circ}$.

Se $\theta_{1}$ existe e $\left|\theta_{1}\right| \leq \theta<90^{\circ}$, então:

$\theta=\frac{\operatorname{arcsen}\left(\frac{2 \cdot n_{x y}}{f_{c d 1} \cdot h}\right)}{2}$

Se $\theta_{1}$ existe e $\left|\theta_{2}\right|<\theta<\left|\theta_{1}\right|$ ou se $\theta_{1}$ não existe e $\left|\theta_{2}\right|<\theta<90^{\circ}$, então: 
$\theta=\frac{\operatorname{arcsen}\left(\frac{2 \cdot n_{x y}}{\frac{f_{c d 1} \cdot h}{0,8+170 \cdot\left[\frac{2 \cdot \varepsilon_{y d}+2 \% \cdot(1+\cos 2 \theta)}{(1-\cos 2 \theta)}\right]}}\right)}{2}$

$E$, finalmente, se $\left|\theta^{*}\right|<\theta \leq\left|\theta_{2}\right|$, então:

$\theta=\frac{\operatorname{arcsen}\left(\frac{2 \cdot n_{x y}}{f_{c d 2} \cdot h}\right)}{2}$

Para o caso II, agora $\varepsilon_{y}=\varepsilon_{y d}$. Assim, com $\varepsilon_{2}=\varepsilon_{c}^{\prime}$ e $\theta$, é possível encontrar o valor de $\varepsilon_{1}$ e então de $\varepsilon_{x}$. Conforme demonstração análoga no item 4.3, tem-se que:

$\varepsilon_{x}=\frac{2 \cdot \varepsilon_{y d}+2 \% 0 \cdot(1+\cos 2 \theta)}{(1-\cos 2 \theta)}-2 \% 0+-\varepsilon_{y d}$

Calculam-se as forças nas armaduras através das equações 2.1 e 2.2.

$$
\begin{aligned}
& n_{s x}=n_{x}+n_{x y} \cdot \operatorname{tg} \theta \\
& n_{s y}=n_{y}+n_{x y} \cdot \operatorname{cotg} \theta
\end{aligned}
$$

As armaduras são dadas por:

$$
\begin{aligned}
& a_{s x}=\frac{n_{s x}}{\sigma_{x}}=\frac{n_{s x}}{E \cdot \varepsilon_{x}} \\
& a_{s y}=\frac{n_{s y}}{\sigma_{y}}=\frac{n_{s y}}{E \cdot \varepsilon_{y}}=\frac{n_{s y}}{E \cdot \varepsilon_{y d}}=\frac{n_{s y}}{f_{y d}}
\end{aligned}
$$




\section{DIMENSIONAMENTO DAS ARMADURAS DE COMPRESSÃO PARA O CASO IV}

O caso IV se difere dos casos II e III apresentados até aqui porque ele não tem a mesma problemática para a consideração da resistência do concreto. Como neste caso a chapa está em estado biaxial de compressão, a resistência do concreto poderia ser até maior que o valor de $f_{c d 1}$, conforme recomendação do CEB (1990) e apresentado no item 2.1. Entretanto, para este estudo e a favor da segurança a resistência do concreto a ser considerada será igual $\mathrm{f}_{\mathrm{cd} 1}$.

A formulação a ser apresentada tem como objetivo dimensionar armaduras nas direções $\mathrm{x}$ e y para chapas em que o concreto não atende ao critério de resistência expresso no item 2.3.5.4.

\subsection{Hipóteses do problema}

Primeiramente, admite-se que o concreto estará em seu limite de tensão no ELU. Outra hipótese do problema é que a chapa está sempre em estado de compressão biaxial, ou seja, a inclusão de armaduras que levem ao surgimento de trações na chapa é um caso que não está contemplado pela formulação deste estudo. Assim, tem-se como hipóteses.

1. A resistência à tração do concreto é desprezada

2. É considerada perfeita aderência entre a armadura e o concreto

3. A chapa sempre está em estado biaxial de compressão.

4. A direção das deformações principais coincide com a direção das tensões principais

5. A resistência do concreto à compressão é dada por $\mathrm{f}_{\mathrm{cd} 1}$.

6. A deformação na direção principal de maior compressão é igual à deformação que resulta na tensão de pico do concreto $\left(\varepsilon_{2}=\varepsilon_{c}^{\prime}\right)$. Assim, está tensão é igual a resistência do concreto à compressão $\left(\sigma_{c}=f_{c d 1}\right)$. 


\subsection{Equações de equilíbrio}

Está demonstração pode ser vista também em Jazra (2008). Dada uma chapa submetida a um estado biaxial de tensão onde há armaduras posicionadas nas direções $x$ e y. Efetuando-se o equilíbrio de um trecho de chapa em que uma das faces tem comprimento unitário e está perpendicular a direção principal de maior tensão de compressão conforme a Figura 5.1, tem-se que:

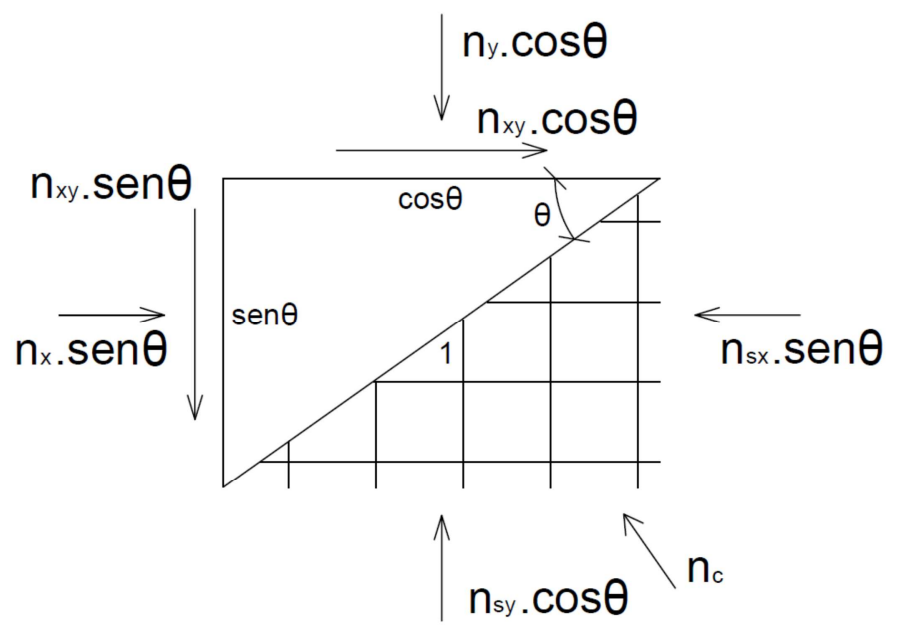

Figura 5.1 - Equilíbrio da chapa em um trecho de chapa com uma das faces perpendicular a direção principal de maior tensão de compressão

$\sum F_{x}=0$

$-n_{x} \cdot \operatorname{sen} \theta+n_{x y} \cdot \cos \theta+n_{s x} \cdot \operatorname{sen} \theta-n_{c} \cdot \operatorname{sen} \theta=0$

$n_{c} \cdot \operatorname{sen} \theta=-n_{x} \cdot \operatorname{sen} \theta+n_{x y} \cdot \cos \theta+n_{s x} \cdot \operatorname{sen} \theta$

$n_{c}=-n_{x}+n_{x y} \cdot \operatorname{cotg} \theta+n_{s x}$

$\operatorname{cotg} \theta=\frac{n_{c}+n_{x}-n_{s x}}{n_{x y}}$

Agora, efetuando-se o equilíbrio na direção y, tem-se que:

$\sum F_{y}=0$

$n_{y} \cdot \cos \theta-n_{x y} \cdot \operatorname{sen} \theta-n_{s y} \cdot \cos \theta+n_{c} \cdot \cos \theta=0$

$n_{c} \cdot \cos \theta=-n_{y} \cdot \cos \theta+n_{x y} \cdot \operatorname{sen} \theta+n_{s y} \cdot \cos \theta$

$n_{c}=n_{s y}-n_{y}+n_{x y} \cdot \operatorname{tg} \theta$ 
Substituindo 5.2 em 5.3, tem-se que:

$$
\begin{aligned}
& n_{c}=n_{s y}-n_{y}+n_{x y} \cdot \operatorname{tg} \theta \\
& n_{c}=n_{s y}-n_{y}+\frac{n_{x y}{ }^{2}}{n_{c}+n_{x}-n_{s x}} \\
& \left(n_{c}+n_{x}-n_{s x}\right) \cdot n_{c}=\left(n_{c}+n_{x}-n_{s x}\right) \cdot n_{s y}-\left(n_{c}+n_{x}-n_{s x}\right) \cdot n_{y}+n_{x y}{ }^{2} \\
& n_{c}{ }^{2}+n_{x} \cdot n_{c}-n_{s x} \cdot n_{c}=n_{c} \cdot n_{s y}+n_{x} \cdot n_{s y}-n_{s x} \cdot n_{s y}-n_{c} \cdot n_{y}-n_{x} \cdot n_{y}+n_{s x} \cdot n_{y}+n_{x y}{ }^{2} \\
& n_{c}{ }^{2}+n_{x} \cdot n_{c}-n_{s x} \cdot n_{c}-n_{c} \cdot n_{s y}-n_{x} \cdot n_{s y}+n_{s x} \cdot n_{s y}+n_{c} \cdot n_{y}+n_{x} \cdot n_{y}-n_{s x} \cdot n_{y}-n_{x y}{ }^{2}=0 \\
& n_{c}{ }^{2}+\left(n_{x}-n_{s x}-n_{s y}+n_{y}\right) \cdot n_{c}-n_{x} \cdot n_{s y}+n_{s x} \cdot n_{s y}+n_{x} \cdot n_{y}-n_{s x} \cdot n_{y}-n_{x y}{ }^{2}=0
\end{aligned}
$$

Esta equação é um polinômio do $2^{\circ}$ grau tendo como variável $\mathrm{n}_{\mathrm{c}}$. Resolvendo-o, temse que:

$$
\begin{aligned}
& \Delta=\left(\left(n_{x}-n_{s x}\right)+\left(n_{y}-n_{s y}\right)\right)^{2}-4 .\left(-n_{x} \cdot n_{s y}+n_{s x} \cdot n_{s y}+n_{x} \cdot n_{y}-n_{s x} \cdot n_{y}-n_{x y}{ }^{2}\right) \\
& \Delta=\left(n_{x}-n_{s x}\right)^{2}+2 \cdot\left(n_{x}-n_{s x}\right) \cdot\left(n_{y}-n_{s y}\right)+\left(n_{y}-n_{s y}\right)^{2}-4 \cdot\left(n_{x}-n_{s x}\right) \cdot\left(n_{y}-n_{s y}\right) \\
& +4 . n_{x y}^{2} \\
& \Delta=\left(n_{x}-n_{s x}\right)^{2}-2 \cdot\left(n_{x}-n_{s x}\right) \cdot\left(n_{y}-n_{s y}\right)+\left(n_{y}-n_{s y}\right)^{2}+4 \cdot n_{x y}{ }^{2} \\
& \Delta=\left(\left(n_{x}-n_{s x}\right)-\left(n_{y}-n_{s y}\right)\right)^{2}+4 . n_{x y}^{2}
\end{aligned}
$$

Então:

$$
\begin{aligned}
& n_{c}=\frac{-\left(n_{x}-n_{s x}-n_{s y}+n_{y}\right) \pm \sqrt{\left(\left(n_{x}-n_{s x}\right)-\left(n_{y}-n_{s y}\right)\right)^{2}+4 . n_{x y}{ }^{2}}}{2} \\
& n_{c}=-\frac{\left(n_{x}-n_{s x}\right)+\left(n_{y}-n_{s y}\right)}{2} \pm \sqrt{\frac{\left(\left(n_{x}-n_{s x}\right)-\left(n_{y}-n_{s y}\right)\right)^{2}}{4}+n_{x y}{ }^{2}}
\end{aligned}
$$

A solução positiva da equação determina a esforço no concreto na direção 2 e a negativa significa o esforço na direção 1. Portanto:

$$
n_{c}=-\frac{\left(n_{x}-n_{s x}\right)+\left(n_{y}-n_{s y}\right)}{2}+\sqrt{\frac{\left(\left(n_{x}-n_{s x}\right)-\left(n_{y}-n_{s y}\right)\right)^{2}}{4}+n_{x y}{ }^{2}}
$$


$n_{c}^{\prime}=-\frac{\left(n_{x}-n_{s x}\right)+\left(n_{y}-n_{s y}\right)}{2}-\sqrt{\frac{\left(\left(n_{x}-n_{s x}\right)-\left(n_{y}-n_{s y}\right)\right)^{2}}{4}+n_{x y}{ }^{2}}$

Fazendo o equilíbrio de esforços em um trecho de chapa em que uma das faces tem comprimento unitário e está perpendicular a direção principal de menor tensão de compressão conforme a Figura 5.2, tem-se que:

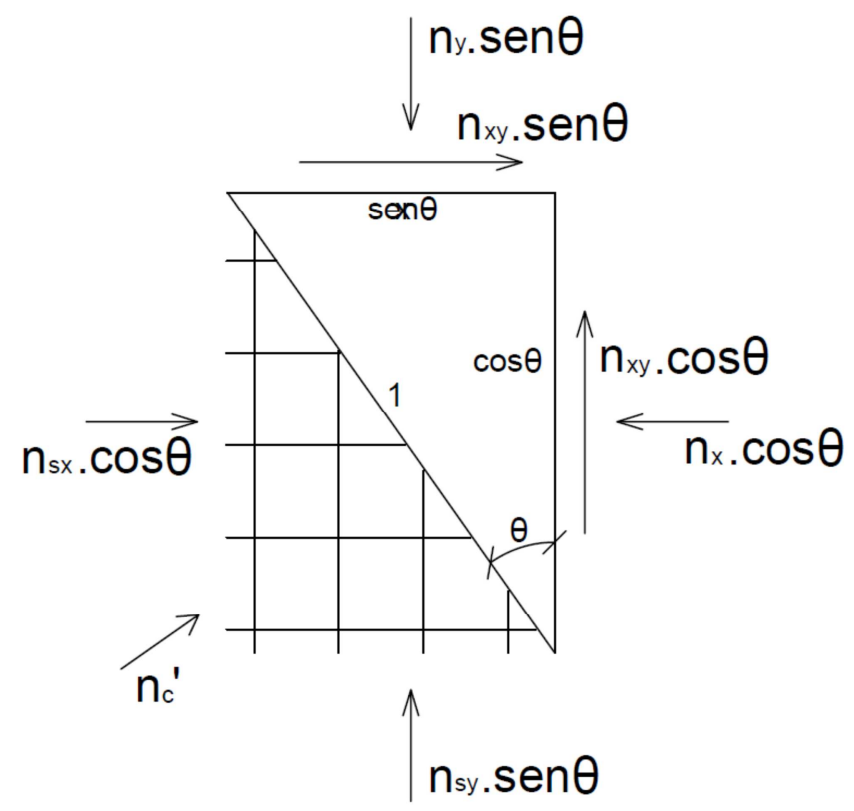

Figura 5.2 - Equilíbrio da chapa em um trecho de chapa com uma das faces perpendicular a direção principal de menos tensão de compressão

$$
\begin{aligned}
& \sum F_{x}=0 \\
& n_{x y} \cdot \operatorname{sen} \theta+n_{x} \cdot \cos \theta-n_{s x} \cdot \cos \theta+n_{c}^{\prime} \cdot \cos \theta=0 \\
& n_{s x} \cdot \cos \theta=n_{x} \cdot \cos \theta+n_{c}^{\prime} \cdot \cos \theta+n_{x y} \cdot \operatorname{sen} \theta \\
& n_{s x}=n_{x}+n_{c}^{\prime}+n_{x y} \cdot \operatorname{tg} \theta \\
& \sum F_{y}=0 \\
& n_{y \cdot} \cdot \operatorname{sen} \theta+n_{x y} \cdot \cos \theta-n_{s y} \cdot \operatorname{sen} \theta+n_{c}^{\prime} \cdot \operatorname{sen} \theta=0 \\
& n_{s y} \cdot \operatorname{sen} \theta=n_{y} \cdot \operatorname{sen} \theta+n_{c}^{\prime} \cdot \operatorname{sen} \theta+n_{x y} \cdot \cos \theta \\
& n_{s y}=n_{y}+n_{c}^{\prime}+n_{x y} \cdot \operatorname{cotg} \theta
\end{aligned}
$$

Substituindo 5.5 em 5.1, tem-se que: 


$$
\begin{aligned}
& n_{c}=-n_{x}+n_{x y \cdot} \cdot \operatorname{cotg} \theta+n_{s x} \\
& n_{c}=-n_{x}+n_{x y} \cdot \operatorname{cotg} \theta+n_{x}+n_{c}^{\prime}+n_{x y} \cdot \operatorname{tg} \theta \\
& n_{c}=n_{x y} \cdot \operatorname{cotg}+n_{c}^{\prime}+n_{x y} \cdot \operatorname{tg} \theta \\
& n_{c}=n_{c}^{\prime}+n_{x y} \cdot(\operatorname{tg} \theta+\operatorname{cotg} \theta)
\end{aligned}
$$

\subsection{Limites de dimensionamento}

Pretende-se definir os casos para os quais é possível dimensionar armaduras de compressão. Dado por hipótese que a chapa sempre está em estado biaxial de compressão, pode-se inferir que:

$n_{c}^{\prime} \geq 0$

Assim, substituindo 5.7 em 5.8, tem-se que:

$$
\begin{aligned}
& n_{c}-n_{x y} \cdot(\operatorname{tg} \theta+\operatorname{cotg} \theta) \geq 0 \\
& n_{c}-\frac{2 \cdot n_{x y}}{\operatorname{sen} 2 \theta} \geq 0 \\
& -2 \cdot n_{x y} \geq-n_{c} \cdot \operatorname{sen} 2 \theta \\
& \frac{-2 \cdot n_{x y}}{n_{c}} \geq-\operatorname{sen} 2 \theta \\
& -\operatorname{sen} 2 \theta \leq-\frac{2 \cdot n_{x y}}{n_{c}} \\
& \operatorname{sen} 2 \theta \geq \frac{2 \cdot n_{x y}}{n_{c}}
\end{aligned}
$$

Como por hipótese $\sigma_{c}=f_{c d 1} e$, portanto $n_{c}=f_{c d 1} \cdot h$, então:

$$
\operatorname{sen} 2 \theta \geq \frac{2 . n_{x y}}{f_{c d 1} \cdot h}
$$


Se aplicarmos módulo, tem-se que:

$0 \leq|\operatorname{sen} 2 \theta| \leq 1$

Portanto:

$$
\begin{aligned}
& 1 \geq \frac{2 \cdot\left|n_{x y}\right|}{f_{c d 1} \cdot h} \\
& \left|n_{x y}\right| \leq \frac{f_{c d 1} \cdot h}{2}
\end{aligned}
$$

A equação 5.10 expressão um limite absoluto para $n_{x y}$.

Isto posto, como as hipóteses definem apenas uma deformação fixa, consequentemente há infinitas soluções dentro de um intervalo. O parâmetro que define este intervalo será $\theta$. Assim, é interessante delimitar quais os ângulos $\theta$ que são possíveis de serem atribuídos ao problema. Desta forma, retomando a equação 5.9, tem-se que:

$\operatorname{sen} 2 \theta \geq \frac{2 . n_{x y}}{f_{c d 1} \cdot h}$

Se transformarmos a inequação 5.9 em equação, a solução para ela com $0^{\circ} \leq \theta \leq$ 90ํassume dois valores dados por:

$\theta_{\mathrm{c} 1}=\frac{\operatorname{arcsen}\left(\frac{2 \cdot \mathrm{n}_{\mathrm{xy}}}{\mathrm{f}_{\mathrm{cd} 1} \cdot \mathrm{h}}\right)}{2} \quad$ para $0^{\circ} \leq \theta \leq 45^{\circ}$

$\theta_{\mathrm{c} 2}=\frac{\operatorname{arcsen}\left(\frac{2 \cdot \mathrm{n}_{\mathrm{xy}}}{\mathrm{f}_{\mathrm{cd} 1 \cdot \mathrm{h}}}\right)}{2} \quad$ para $45^{\circ} \leq \theta \leq 90^{\circ}$

Para que a inequação 5.9 seja respeitada, os valores de $\theta$ devem estar entre $\theta_{c 1}$ e $\theta_{\mathrm{c} 2}$ obrigatoriamente. Em caso de $\mathrm{n}_{\mathrm{xy}}$ negativos, $-90^{\circ} \leq \theta \leq 0^{\circ}$ as mesmas equações são válidas, porém com os sinais dos ângulos do domínio invertido. 
$\theta_{\mathrm{c} 1}=\frac{\operatorname{arcsen}\left(\frac{2 \cdot \mathrm{n}_{\mathrm{xy}}}{\mathrm{f}_{\mathrm{cd} 1} \cdot \mathrm{h}}\right)}{2} \quad$ para $-90^{\circ} \leq \theta \leq-45^{\circ}$

$\theta_{\mathrm{c} 2}=\frac{\operatorname{arcsen}\left(\frac{2 \cdot \mathrm{n}_{\mathrm{xy}}}{\mathrm{f}_{\mathrm{cd} 1} \cdot \mathrm{h}}\right)}{2} \quad$ para $-45^{\circ} \leq \theta \leq 0^{\circ}$

Assim:

$\theta_{c 1} \leq \theta \leq \theta_{c 2}$

Isto posto, além deste critério, como por hipótese a chapa sempre está em estado biaxial de tensão, as deformações em qualquer direção sempre tem sinal negativo. Desta forma, para não se obter armaduras com sinal negativo, o que é uma incongruência, as forças nas armaduras devem ser negativas também. Sendo assim, tem-se que:

$n_{s x} \leq 0$

$n_{s y} \leq 0$

Substituindo 5.1 em 5.14, tem-se que:

$$
\begin{aligned}
& n_{c}=-n_{x}+n_{x y} \cdot \operatorname{cotg} \theta+n_{s x} \\
& n_{x}+n_{c}-n_{x y} \cdot \operatorname{cotg} \theta \leq 0 \\
& -\operatorname{cotg} \theta \leq \frac{-\left(n_{x}+n_{c}\right)}{n_{x y}} \\
& \operatorname{cotg} \theta \geq \frac{n_{x}+n_{c}}{n_{x y}} \\
& \operatorname{tg} \theta \leq \frac{n_{x y}}{n_{x}+n_{c}} \\
& \theta \leq \operatorname{arctg}\left(\frac{n_{x y}}{n_{x}+n_{c}}\right) \\
& \theta_{x}=\operatorname{arctg}\left(\frac{n_{x y}}{n_{x}+n_{c}}\right)
\end{aligned}
$$


De maneira análoga substituindo 5.3 em 5.15, tem-se que:

$$
\begin{aligned}
& n_{c}=n_{s y}-n_{y}+n_{x y} \cdot \operatorname{tg} \theta \\
& n_{y}-n_{x y} \cdot \operatorname{tg} \theta+n_{c} \leq 0 \\
& -\operatorname{tg} \theta \leq \frac{-\left(n_{c}+n_{y}\right)}{n_{x y}} \\
& \operatorname{tg} \theta \geq \frac{n_{c}+n_{y}}{n_{x y}} \\
& \theta \geq \operatorname{arctg}\left(\frac{n_{c}+n_{y}}{n_{x y}}\right) \\
& \theta_{y}=\operatorname{arctg}\left(\frac{n_{c}+n_{y}}{n_{x y}}\right)
\end{aligned}
$$

\subsection{Dimensionamento das armaduras}

O método a ser apresentado foi baseado daquele de Jazra (2008). Primeiramente os esforços para o qual chapa está submetida devem respeitar a equação 5.10. Depois de verificado este critério, deve-se arbitrar um valor de $\theta$ tal que respeite os limites impostos no item 5.3. Dos infinitos $\theta$ possíveis, um valor resultará na armadura mínima. Este será simbolizado por $\theta_{\text {amin. }}$ Com isto, retomando a expressão 5.1, calcula-se:

$$
\begin{aligned}
& n_{c}=-n_{x}+n_{x y} \cdot \operatorname{cotg} \theta+n_{s x} \\
& n_{s x}=n_{c}+n_{x}-n_{x y} \cdot \operatorname{cotg} \theta
\end{aligned}
$$

E a partir da expressão 5.3, tem-se que:

$$
\begin{aligned}
& n_{c}=n_{s y}-n_{y}+n_{x y} \cdot \operatorname{tg} \theta \\
& n_{s y}=n_{c}+n_{y}-n_{x y} \cdot \operatorname{tg} \theta
\end{aligned}
$$

Com os valores de $n_{s x}$ e $n_{s y}$, é possível calcular o valor de $n_{c}$ ' através da expressão 5.5 . 
$n_{c}^{\prime}=-\frac{\left(n_{x}-n_{s x}\right)+\left(n_{y}-n_{s y}\right)}{2}-\sqrt{\frac{\left(\left(n_{x}-n_{s x}\right)-\left(n_{y}-n_{s y}\right)\right)^{2}}{4}+n_{x y}{ }^{2}}$

Como a direção principal das tensões é a mesma das deformações por hipótese, a tensão $\mathrm{n}_{\mathrm{c}}$ ' produz a deformação $\varepsilon_{1}$. Estes termos se relacionam através da expressão 3.4 que configura o diagrama tensão-deformação para o concreto.

$\sigma_{c}=f_{c 2 \max } \cdot\left[2 \cdot\left(\frac{\varepsilon_{2}}{\varepsilon_{c}^{\prime}}\right)-\left(\frac{\varepsilon_{2}}{\varepsilon_{c}^{\prime}}\right)^{2}\right]$

Sendo que sua solução é a equação 3.17.

$\varepsilon_{2}=\varepsilon_{c} \cdot\left(1-\sqrt{1-\frac{\sigma_{c}}{f_{c 2 \max }}}\right)$

Como para o caso IV, $\mathrm{f}_{\mathrm{c} 2 \max }=\mathrm{f}_{\mathrm{cd} 1}$, para $\varepsilon_{1}$ tem-se que:

$$
\begin{aligned}
& \varepsilon_{1}=\varepsilon_{c}^{\prime} \cdot\left(1-\sqrt{1-\frac{\sigma_{c}^{\prime}}{f_{c d 1}}}\right) \\
& \varepsilon_{1}=\varepsilon_{c}^{\prime} \cdot\left(1-\sqrt{1-\frac{n_{c}^{\prime}}{f_{c d 1} \cdot h}}\right)
\end{aligned}
$$

Obtendo o valor de $\varepsilon_{1}$, como se conhece o valor de $\varepsilon_{2}=\varepsilon_{c}^{\prime}$ e $\theta$, pode-se calcular os valores das deformações $\varepsilon_{x}$ e $\varepsilon_{y}$ através de 3.12 e 3.13 .

$$
\begin{aligned}
& \varepsilon_{x}=\frac{\varepsilon_{1}+\varepsilon_{2}}{2}+\left(\frac{\varepsilon_{1}-\varepsilon_{2}}{2}\right) \cdot \cos 2 \theta \\
& \varepsilon_{y}=\frac{\varepsilon_{1}+\varepsilon_{2}}{2}-\left(\frac{\varepsilon_{1}-\varepsilon_{2}}{2}\right) \cdot \cos 2 \theta
\end{aligned}
$$

Com isto, dimensionam-se as armaduras: 
$a_{s x}=\frac{n_{s x}}{\sigma_{x}}=\frac{n_{s x}}{E_{c s} \cdot \varepsilon_{x}}$

$a_{s y}=\frac{n_{s y}}{\sigma_{y}}=\frac{n_{s y}}{E_{c s} \cdot \varepsilon_{y}}$

As armaduras dimensionadas não são necessariamente as mínimas. Portanto, devese realizar algumas tentativas para encontrar este mínimo.

\subsection{Exemplo de dimensionamento}

Este exemplo aplica os conceitos mostrados no neste capítulo. Será admitidos $\mathrm{f}_{\mathrm{ck}}=$ $25 \mathrm{MPa}, \mathrm{h}=12 \mathrm{~cm}$, armaduras CA50 e serão adotados os coeficientes de segurança recomendados pela NBR 6118. Todos os esforços são de cálculo.

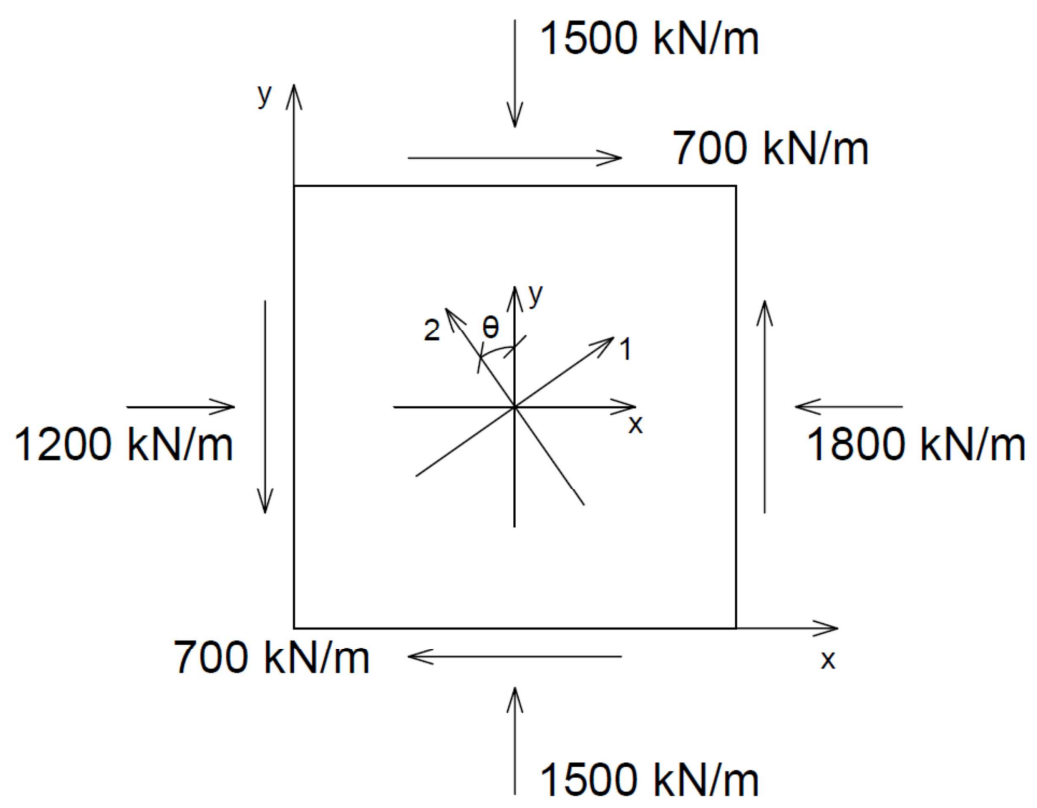

Figura 5.3 - Exemplo 5

$h=12 \mathrm{~cm}$

$f_{c k}=25 \mathrm{MPa}$

$n_{x}=-1200 \mathrm{kN} / \mathrm{m}$

$n_{y}=-1500 \mathrm{kN} / \mathrm{m}$

$n_{x y}=700 \mathrm{kN} / \mathrm{m}$ 
Primeiramente, deve-se verificar em qual caso de dimensionamento essa chapa se encontra. Assim, retomando as expressões 2.11 e 2.12, tem-se que:

$$
\begin{aligned}
& n_{s x}=n_{x}+\left|n_{x y}\right| \\
& n_{s y}=n_{y}+\left|n_{x y}\right| \\
& n_{s x}=-1200+|700|=-500 \\
& n_{s y}=-1500+|700|=-800
\end{aligned}
$$

Como as inequações 2.31 e 2.32 foram satisfeitas, a chapa se encaixa no caso IV de dimensionamento.

$$
\begin{aligned}
& n_{s x}=n_{x}+\left|n_{x y}\right| \leq 0 \\
& n_{s y}=n_{y}+\left|n_{x y}\right| \leq 0
\end{aligned}
$$

Assim, deve-se agora apenas verificar se a maior tensão de compressão no concreto respeita o limite de $\mathrm{f}_{\mathrm{cd} 1}$, de acordo com a expressão 2.41.

$$
\begin{aligned}
& \sigma_{c}=\frac{n_{c}}{h} \leq f_{c d 1} \\
& \left(-\frac{n_{x}+n_{y}}{2}+\sqrt{\frac{\left(n_{x}-n_{y}\right)^{2}}{4}+n_{x y}^{2}}\right) \cdot \frac{1}{h} \leq 0,85 \cdot\left(1-\frac{f_{c k}}{250}\right) \cdot f_{c d}
\end{aligned}
$$

Assim, tem-se que:

$$
f_{c d 1}=0,85 \cdot\left(1-\frac{f_{c k}}{250}\right) \cdot f_{c d}=0,85 \cdot\left(1-\frac{25}{250}\right) \cdot \frac{25000}{1,4}=13660,7 \mathrm{kN} / \mathrm{m}^{2}
$$

E:

$$
\frac{n_{c}}{h}=\left(-\frac{-1200-1500}{2}+\sqrt{\frac{(-1200+1500)^{2}}{4}+700^{2}}\right) \cdot \frac{1}{0,12}=17215,8 \mathrm{kN} / \mathrm{m}^{2}
$$


Como:

$\frac{n_{c}}{h}>f_{c d 1}$

Então, a maior tensão de compressão no concreto não respeita a limite de resistência estabelecido. Portanto, deve-se avaliar a possibilidade de adoção de armaduras de compressão. Primeiramente, verifica-se se o esforço de cisalhamento atende ao critério expresso pela equação 5.10 .

$\left|n_{x y}\right| \leq \frac{f_{c d 1} \cdot h}{2}$

$|700| \leq \frac{13660,7 \cdot 0,12}{2}=819,64 \mathrm{kN} / \mathrm{m}$

Assim, como $\mathrm{n}_{\mathrm{xy}}$ está abaixo do limite, então é possível dimensionar armaduras de compressão. Desta forma, se deve encontrar os limites $\theta_{c 1}$ e $\theta_{c 2}$ através das expressões 5.11 e 5.12 .

$\theta_{c 1}=\frac{\operatorname{arcsen}\left(\frac{2 \cdot n_{x y}}{f_{c d 1} \cdot h}\right)}{2} \quad$ para $0^{\circ} \leq \theta \leq 45^{\circ}$

$\theta_{c 2}=\frac{\operatorname{arcsen}\left(\frac{2 \cdot n_{x y}}{f_{c d 1} \cdot h}\right)}{2} \quad$ para $45^{\circ} \leq \theta \leq 90^{\circ}$

Assim, tem-se que:

$$
\begin{aligned}
& \theta_{c 1}=\frac{\operatorname{arcsen}\left(\frac{2.700}{13660,7 \cdot 0,12}\right)}{2}=29,33^{\circ} \\
& \theta_{c 2}=\frac{\operatorname{arcsen}\left(\frac{2.700}{13660,7 \cdot 0,12}\right)}{2}=60,67^{\circ}
\end{aligned}
$$

Prosseguindo, calcula-se os limites $\theta_{x}$ e $\theta_{y}$ através das equações 5.17 e 5.19 
$\theta_{x}=\operatorname{arctg}\left(\frac{n_{x y}}{n_{x}+n_{c}}\right)$

$\theta_{y}=\operatorname{arctg}\left(\frac{n_{c}+n_{y}}{n_{x y}}\right)$

Assim, como por hipótese $n_{c}=f_{c d 1} \cdot h$, tem-se que:

$$
\begin{aligned}
& \theta_{x}=\operatorname{arctg}\left(\frac{700}{-1200+1639,29}\right)=57,89^{\circ} \\
& \theta_{y}=\operatorname{arctg}\left(\frac{1639,29-1500}{700}\right)=11,25^{\circ}
\end{aligned}
$$

Retomando os critérios 5.13, 5.16 e 5.18, tem-se que:

$\theta_{c 1} \leq \theta \leq \theta_{c 2}$

$\theta \leq \operatorname{arctg}\left(\frac{n_{x y}}{n_{x}+n_{c}}\right)=\theta_{x}$

$\theta \geq \operatorname{arctg}\left(\frac{n_{c}+n_{y}}{n_{x y}}\right)=\theta_{y}$

Então:

$29,33^{\circ} \leq \theta \leq 60,67^{\circ}$

$\theta \leq 57,89^{\circ}$

$\theta \geq 11,25^{\circ}$

Portanto:

$29,33^{\circ} \leq \theta \leq 57,89^{\circ}$

Assim, arbitrando um $\theta$ neste intervalo calcula-se as armaduras. Desta forma, fixando, por exemplo, $\theta=40^{\circ}$ e retomando as equações 5.20 e 5.21 , tem-se que:

$n_{s x}=n_{c}+n_{x}-n_{x y} \cdot \operatorname{cotg} \theta$ 
$n_{s y}=n_{c}+n_{y}-n_{x y} \cdot \operatorname{tg} \theta$

Assim, tem-se que:

$n_{s x}=1639,29-1200-700 \cdot \operatorname{cotg} 40^{\circ}=-394,9 \mathrm{kN} / \mathrm{m}$

$n_{s y}=1639,29-1500-700 \cdot \operatorname{tg} 40^{\circ}=-448,1 \mathrm{kN} / \mathrm{m}$

Com isto, calcula-se $\mathrm{n}_{\mathrm{c}}$ ' através da expressão 5.5.

$$
\begin{gathered}
n_{c}^{\prime}=-\frac{\left(n_{x}-n_{s x}\right)+\left(n_{y}-n_{s y}\right)}{2}-\sqrt{\frac{\left(\left(n_{x}-n_{s x}\right)-\left(n_{y}-n_{s y}\right)\right)^{2}}{4}+n_{x y}{ }^{2}} \\
n_{c}^{\prime}=-\frac{(-1200+394,9)+(-1500+448,1)}{2} \\
-\sqrt{\frac{((-1200+394,9)-(-1500+448,1))^{2}}{4}+700^{2}}
\end{gathered}
$$

$n_{c}^{\prime}=217,7 \mathrm{kN} / \mathrm{m}$

Agora, determina-se $\varepsilon_{1}$ pela equação 5.22 .

$$
\begin{aligned}
& \varepsilon_{1}=\varepsilon_{c}^{\prime} \cdot\left(1-\sqrt{1-\frac{n_{c}^{\prime}}{f_{c d 1} \cdot h}}\right) \\
& \varepsilon_{1}=-2 \% 0 \cdot\left(1-\sqrt{1-\frac{217,7}{1639,29}}\right)=-0,137 \% 0
\end{aligned}
$$

Tendo $\varepsilon_{1}$, pode-se calcular as $\varepsilon_{x}$ e $\varepsilon_{y}$.

$$
\begin{aligned}
& \varepsilon_{x}=\frac{\varepsilon_{1}+\varepsilon_{2}}{2}+\left(\frac{\varepsilon_{1}-\varepsilon_{2}}{2}\right) \cdot \cos 2 \theta \\
& \varepsilon_{y}=\frac{\varepsilon_{1}+\varepsilon_{2}}{2}-\left(\frac{\varepsilon_{1}-\varepsilon_{2}}{2}\right) \cdot \cos 2 \theta
\end{aligned}
$$

Assim, tem-se que: 
$\varepsilon_{x}=\frac{-0,137-2}{2}+\left(\frac{-0,137+2}{2}\right) \cdot \cos \left(2.40^{\circ}\right)=-0,907 \% 0$

$\varepsilon_{y}=\frac{-0,137-2}{2}-\left(\frac{-0,137+2}{2}\right) \cdot \cos \left(2 \cdot 40^{\circ}\right)=-1,230 \% 0$

Desta forma, obtêm-se as armaduras:

$a_{s x}=\frac{n_{s x}}{\sigma_{x}}=\frac{n_{s x}}{E_{c s} \cdot \varepsilon_{x}}=\frac{-394,9}{21000 \cdot(-0,907 \% 0)}=20,73 \mathrm{~cm}^{2} / \mathrm{m}$

$a_{s y}=\frac{n_{s y}}{\sigma_{y}}=\frac{n_{s y}}{E_{c s} \cdot \varepsilon_{y}}=\frac{-448,1}{21000 \cdot(-1,230 \% 0)}=17,34 \mathrm{~cm}^{2} / \mathrm{m}$

$a_{t}=a_{s x}+a_{s y}=38,07 \mathrm{~cm}^{2} / \mathrm{m}$

Repetindo o mesmo processo para vários ângulos $\theta$, obtém-se o gráfico da Figura 5.4 .

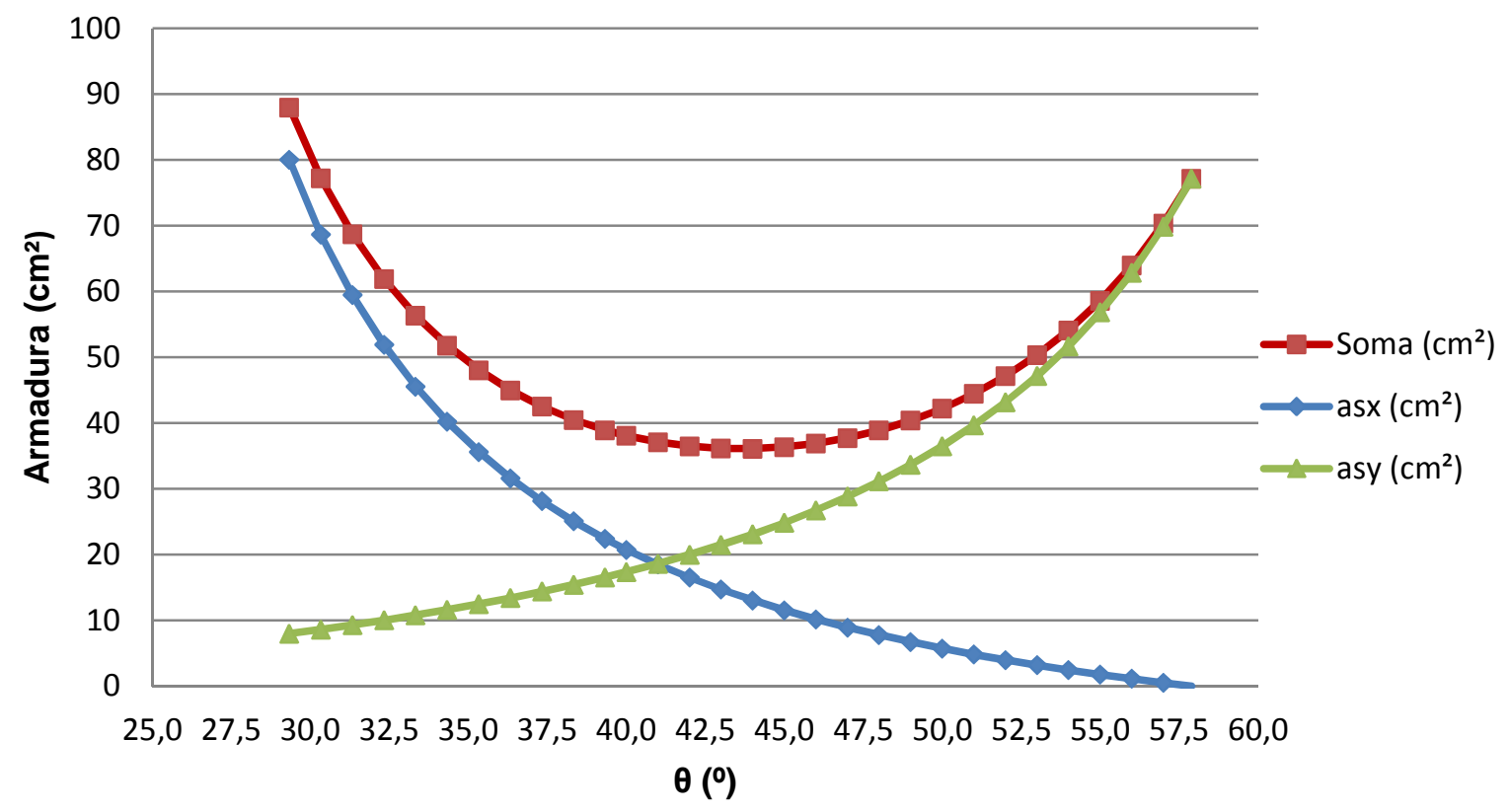

Figura 5.4 - Armadura $x \theta$ para o exemplo 5.5

A partir deste processo, chega-se ao resultado para armadura mínima de:

$$
\begin{aligned}
& \theta_{\text {amin }}=43,62^{\circ} \\
& a_{t}=a_{s x}+a_{s y}=13,64+22,45=36,09 \mathrm{~cm}^{2} / \mathrm{m}
\end{aligned}
$$




\section{DISCUSSÃO DOS RESULTADOS}

Neste capítulo pretende-se mostrar como cada esforço solicitante influencia no consumo das armaduras quando utilizados os métodos de dimensionamento apresentados no Capítulo 4 para os casos II e III e no Capítulo 5 para o caso IV, e discutir suas causas.

\subsection{Casos II e III de dimensionamento}

Da mesma forma que no capítulo 4, neste também se analisará apenas o caso III de dimensionamento, sendo que as mesmas ideias aqui colocadas são válidas para o caso II.

Assim, primeiramente, retomando as equações que determinam o ângulo $\theta$ para o dimensionamento da armadura de compressão no caso III, pode-se ver que fixada a resistência característica do concreto, a espessura da chapa e o tipo de aço, $\theta$ fica em função apenas de $n_{x y}$.

$\theta=\frac{\operatorname{arcsen}\left(\frac{2 \cdot n_{x y}}{f_{c d 1} \cdot h}\right)}{2} \quad$ para $0^{\circ}<\theta \leq\left|\theta_{1}\right|$

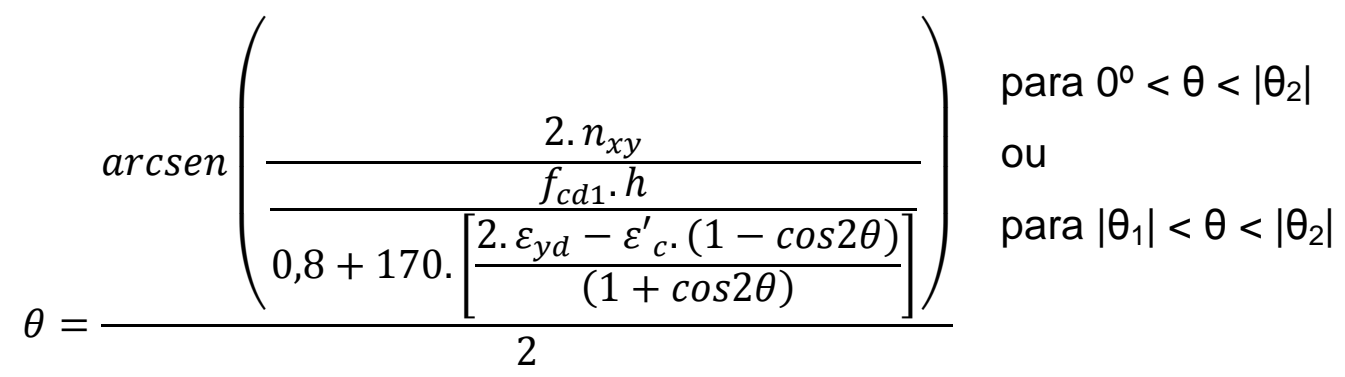

$\theta=\frac{\operatorname{arcsen}\left(\frac{2 . n_{x y}}{f_{c d 2} \cdot h}\right)}{2} \quad$ para $\left|\theta_{2}\right| \leq \theta<\left|\theta^{*}\right|$

Em outras palavras, $\theta$ não depende dos esforços solicitantes $n_{x}$ e $n_{y}$, diferentemente de quando há apenas armadura de tração no caso III. Isso se deve a hipótese de que para o cálculo das armaduras de compressão para o ELU, $\varepsilon_{2}=\varepsilon_{\mathrm{c}}^{\prime}$. Assim tem-se 
uma variável fixa a mais do que no caso de haver somente uma direção armada no caso III.

Olhando pelo lado das armaduras, retomando as equações 2.1 e 2.2, pode-se ver que as forças $n_{s x}$ e $n_{s y}$ dependem linearmente de $n_{x}$ e $n_{y}$ respectivamente. Além disso, como para um dado $\mathrm{n}_{\mathrm{xy}}$ só existirá um ângulo $\theta$ possível, o fator dependente do cisalhamento se manterá constante caso $\mathrm{n}_{\mathrm{xy}}$ for constante.

$$
\begin{aligned}
& n_{s x}=n_{x}+n_{x y} \cdot \operatorname{tg} \theta \\
& n_{s y}=n_{y}+n_{x y} \cdot \operatorname{cotg} \theta
\end{aligned}
$$

Agora, retomando as equações que definem as áreas de armaduras em x e y $4.13 \mathrm{e}$ 4.14. Como por hipótese $\varepsilon_{x}=\varepsilon_{y d}, a_{s x}$ é somente a força $n_{s x}$ dividida por um fator constante. Já $a_{s y}$ depende da deformação $\varepsilon_{y}$. Porém, se $n_{x y}$ for mantido constante, como posto anteriormente, $\theta$ é constante $e$, por consequência, $\varepsilon_{y}$ também é constante.

$$
\begin{aligned}
& a_{s x}=\frac{n_{s x}}{\sigma_{x}}=\frac{n_{s x}}{E_{c s} \cdot \varepsilon_{x}}=\frac{n_{s x}}{E \cdot \varepsilon_{y d}}=\frac{n_{s x}}{f_{y d}} \\
& a_{s y}=\frac{n_{s y}}{\sigma_{y}}=\frac{n_{s y}}{E_{c s} \cdot \varepsilon_{y}}
\end{aligned}
$$

Concluindo, para $\mathrm{n}_{\mathrm{xy}}$ constante, as áreas de armadura dependem linearmente de $n_{x}$ e $n_{y}$, como apresentado pela Figura 6.1 e Figura 6.2.

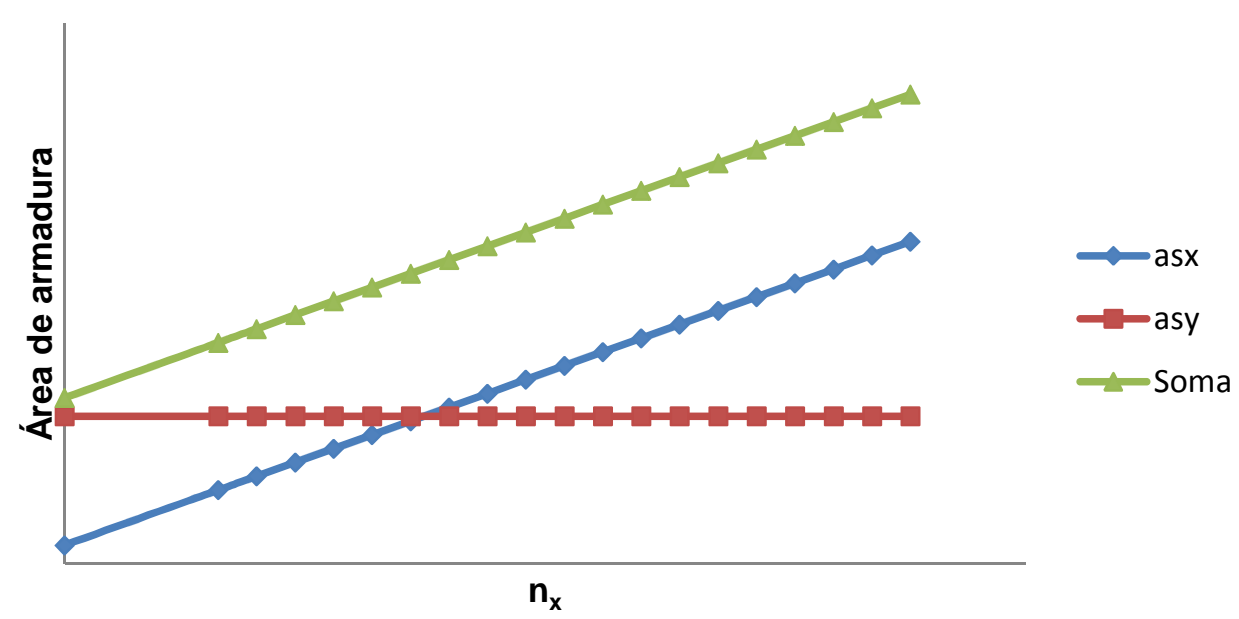

Figura 6.1 - Área de armadura em função do esforço normal $n_{x}$ para o caso III 


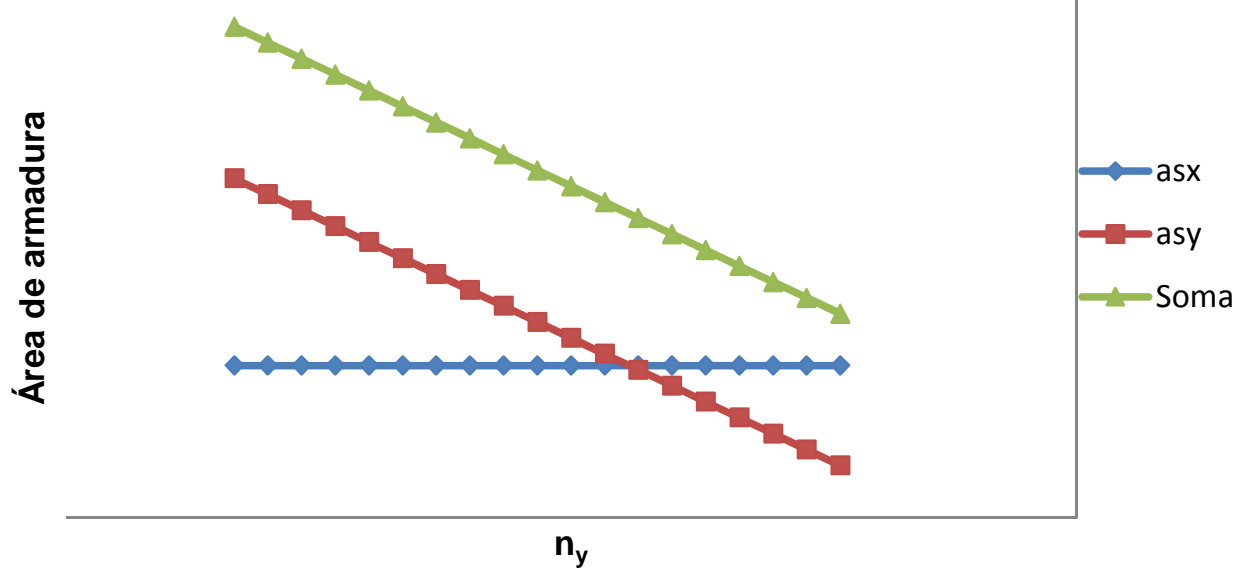

Figura 6.2 - Área de armadura em função do esforço normal ny para o caso III

Quando observado a variação das armaduras em função de $\mathrm{n}_{\mathrm{xy}} \mathrm{o}$ quadro é outro. Se for feito um gráfico das expressões 4.13, 4.14 e 4.15 que determinam a relação entre $n_{x y}$ e $\theta$, então se terá algo semelhante ao apresentado na Figura 6.3.

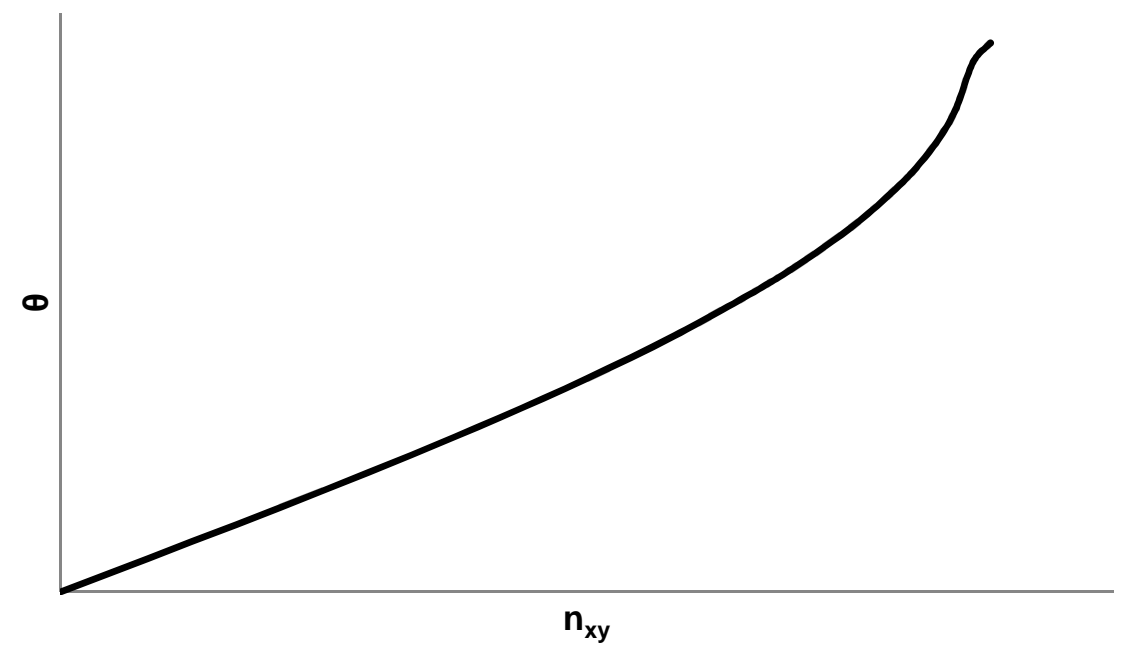

Figura 6.3 - Ângulo $\theta$ em função do esforço de cisalhamento para caso III

Pode se observar que esta função é crescente no domínio. Assim, analisando novamente as expressões 2.1 e 2.2 , tg $(\theta)$ também terá um valor cada vez maior. Como tanto $n_{x y}$ quanto $\operatorname{tg}(\theta)$ aumentam, o fator que inclui o cisalhamento na expressão 2.1 será sempre crescente também. 
Já a $\operatorname{cotg}(\theta)$ terá o seu valor cada vez menor com o aumento de $\mathrm{n}_{\mathrm{xy}}$. Porém, deve se lembrar que para o caso III $n_{y}$ é sempre negativo. Portanto, como o fator $n_{x y} \cdot \operatorname{cotg}(\theta)$ é sempre positivo (item 2.3.3), então, em módulo, $n_{\text {sy }}$ também aumenta de valor.

$n_{s x}=n_{x}+n_{x y} \cdot \operatorname{tg} \theta$

$n_{s y}=n_{y}+n_{x y} \cdot \operatorname{cotg} \theta$

Fazendo um gráfico das expressões 2.1 e 2.2 com $n_{x}$ e $n_{y}$ constantes, obtém-se o gráfico da Figura 6.4.

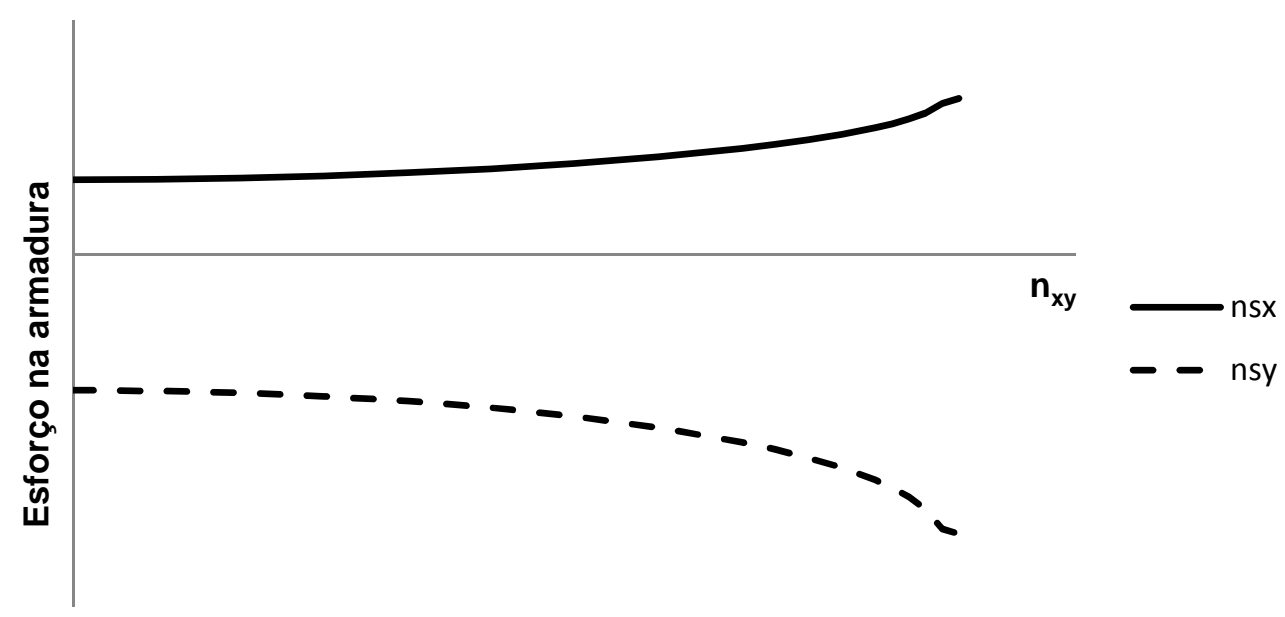

Figura 6.4 - Esforço nas armaduras em função do esforço de cisalhamento para o caso III

Assim, a quantidade de armadura aumenta nas duas direções quanto maior o $\mathrm{n}_{\mathrm{xy}}$. Além disso, outra consequência do aumento de $\theta$ com $n_{x y}$ é que, como $\varepsilon_{x}=\varepsilon_{y d}$ e $\varepsilon_{2}=$ $\varepsilon_{c}^{\prime}, \varepsilon_{y}$ diminui em módulo o seu valor quanto maior $n_{x y}$, como se pode ver pela Figura 6.5 . 


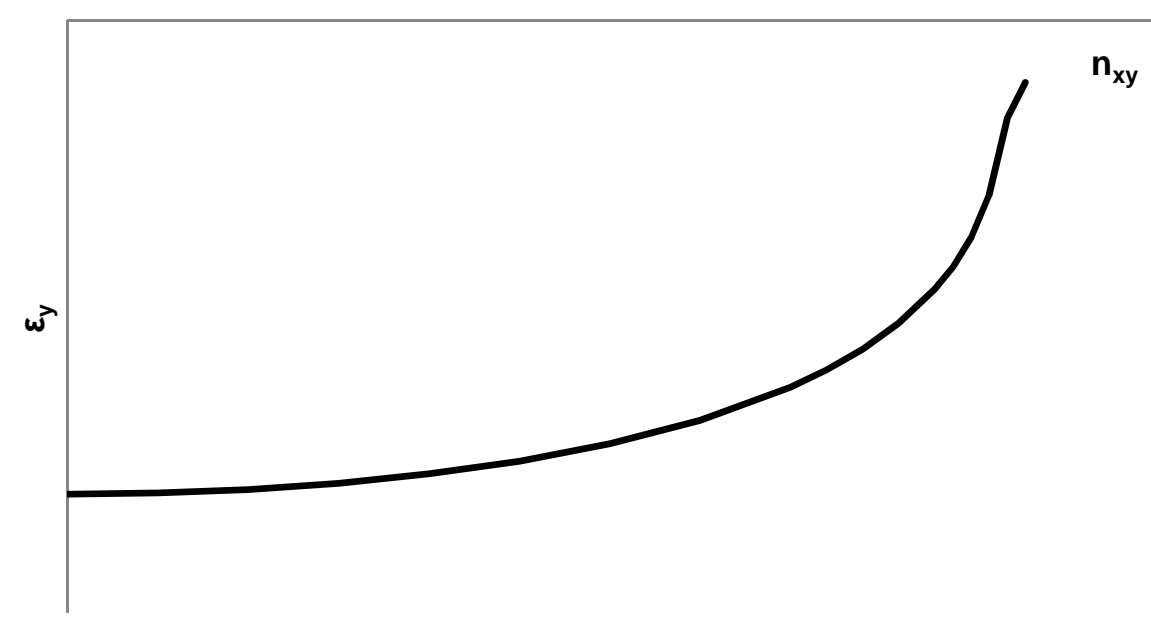

Figura 6.5 - Deformação $\varepsilon_{y}$ em função do esforço de cisalhamente para o caso III

Assim, como quanto menor o valor de $\varepsilon_{y}$, maior a quantidade necessária de armadura em y, a armadura em y fica maior que em $x$ para $\theta$ próximos do limite. No limite, $\varepsilon_{y}$ se aproxima de 0 e $a_{s y}$ tende para 0 infinito. A Figura 6.6 ilustra este comportamento.

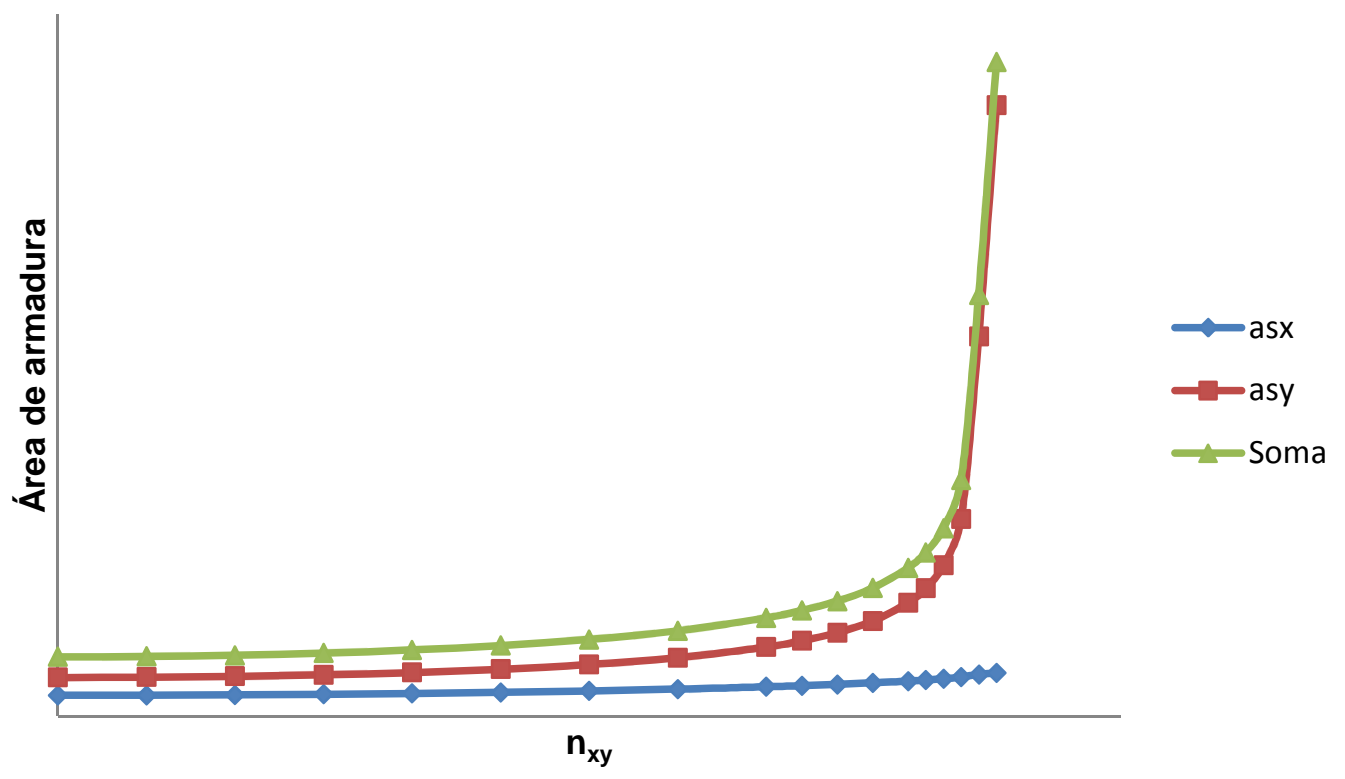

Figura 6.6 - Área de armadura em função do esforço de cisalhamento para o caso III 


\subsection{Caso IV de dimensionamento}

Como para o caso IV a determinação deste ângulo $\theta_{\text {amin }}$ é feita por tentativas, é difícil avaliar como é a relação entre os esforços solicitantes e a quantidade de armadura.

Analisando os esforços normais primeiramente, $n_{x}$ e $n_{y}$, percebe-se que, diferentemente do caso III, eles influem em $\theta_{\text {amin. }}$ Ao se variar apenas $n_{x}$, ou seja, deixando os esforços $n_{y}$ e $n_{x y}$ fixos, obtém-se um gráfico semelhante ao apresentado pela Figura 6.7.

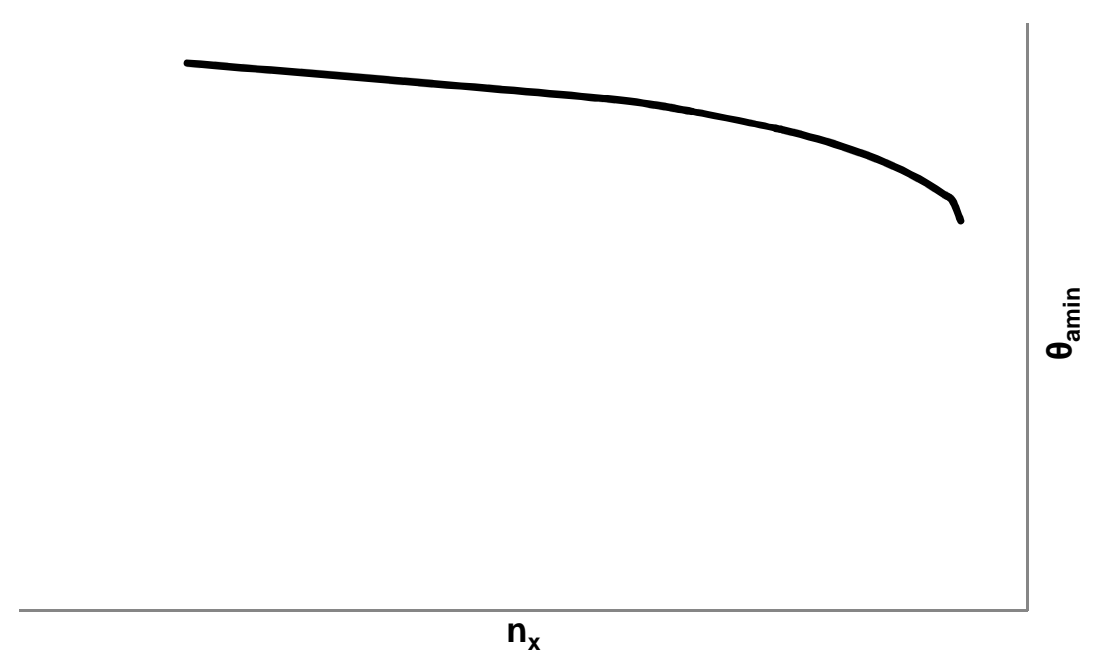

Figura 6.7 - Ângulo $\theta$ em função do esforço normal para caso IV

Isso acontece porque, como explicado item 5.3, diferentemente do caso III, no caso IV somente uma deformação é fixa que é $\varepsilon_{2}=\varepsilon_{c}^{\prime}$. Este fato determina que haja infinitas soluções para o problema dentro de um intervalo. Sendo assim, ao se variar $\theta, \varepsilon_{\mathrm{x}}$ e $\varepsilon_{\mathrm{y}}$ também são alterados.

Assim, a armadura mínima é alcançada para certa combinação de $\varepsilon_{x}$ e $\varepsilon_{y}$ e, portanto para um $\theta_{\text {amin }}$ específico que não necessariamente será igual com a variação do esforço normal $\mathrm{n}_{\mathrm{x}}$.

Devido a este fato, a variação de $n_{x}$ obviamente faz com que as armaduras da direção $x$ mudem, mas também altera aquelas na direção y. As mesmas conclusões são válidas para $n_{y}$. As Figura 6.8 e Figura 6.9 mostram como a variação o esforço 
normal afeta as áreas de armadura em x e y. A Figura 6.10 mostra a variação da área de aço em função do esforço de cisalhamento $\mathrm{n}_{\mathrm{xy}}$.

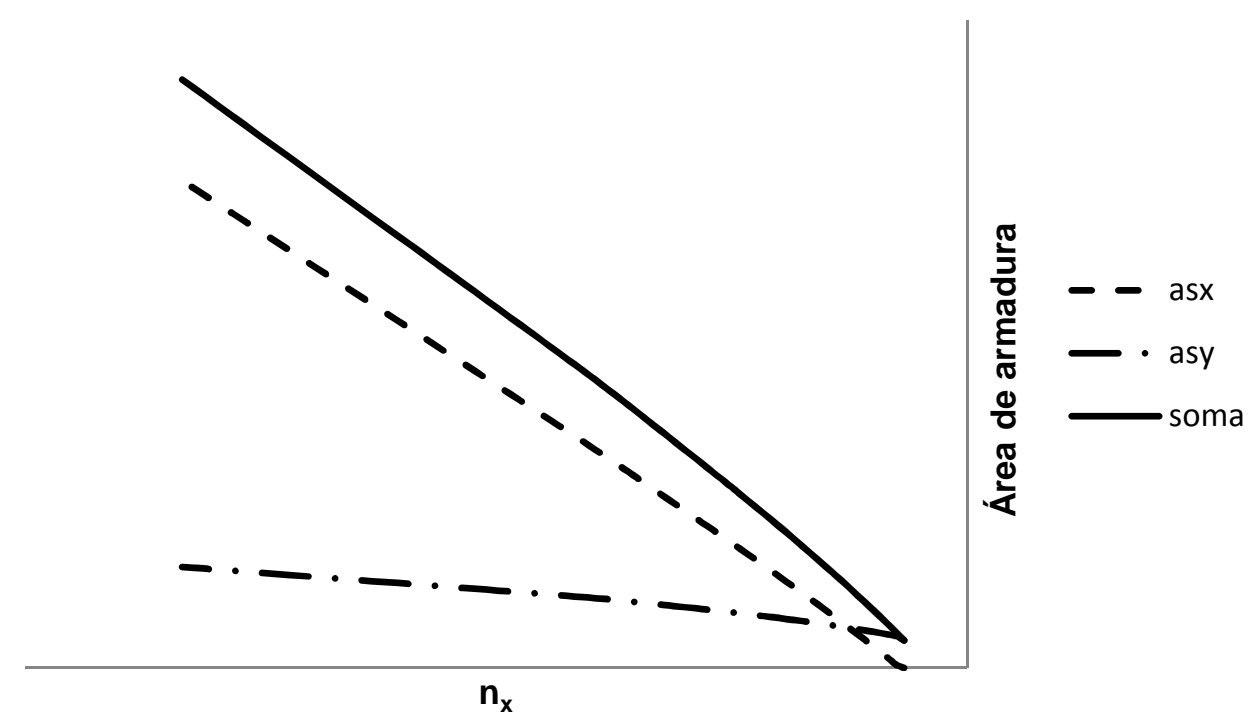

Figura 6.8 - Área de armadura em função do esforço normal $n_{x}$ para o caso IV

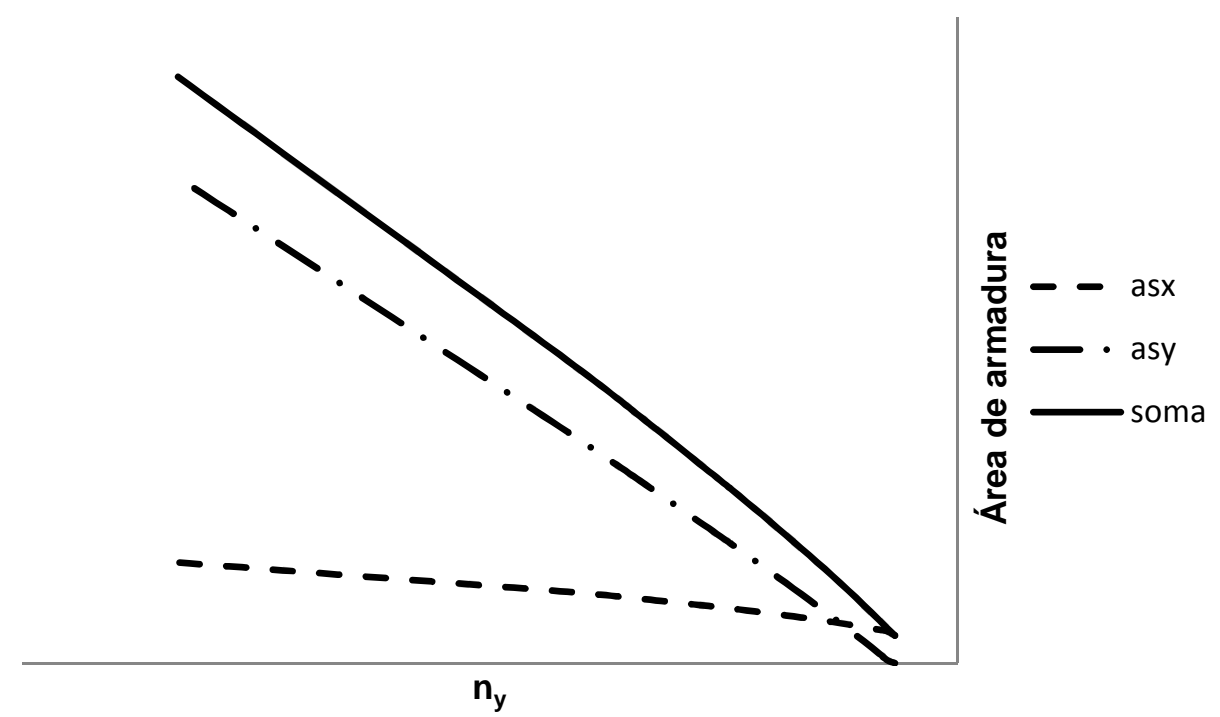

Figura 6.9 - Área de armadura em função do esforço normal $n_{y}$ para o caso IV 


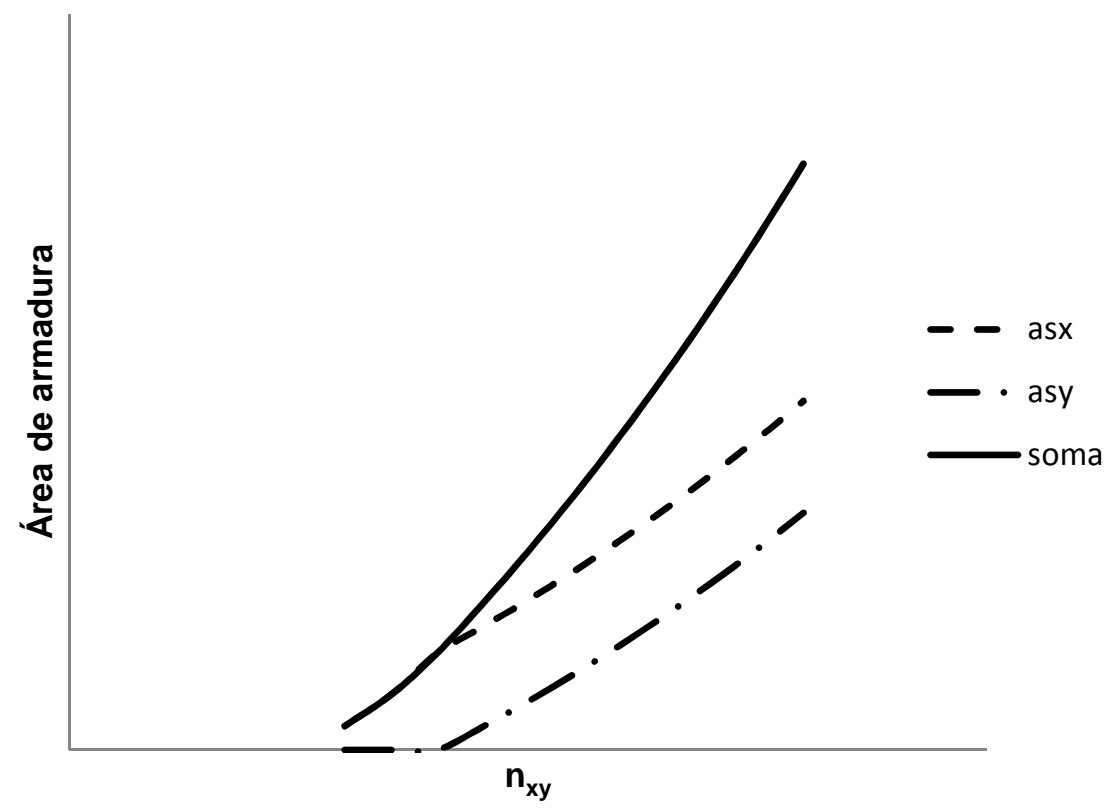

Figura 6.10 - Área de armadura em função do esforço de cisalhamento $\mathrm{n}_{\mathrm{xy}}$ para o caso IV

Agora, tomando novamente como exemplo a mesma chapa do item 5.5. Primeiramente, será encontrado o conjunto de esforços equivalentes (com as mesmas tensões principais) ao do exemplo em 5.5 para vários ângulos $\varphi$. Este ângulo é aquele que a direção principal de compressão faz com o eixo y levando em conta somente os esforços solicitantes. A Tabela 6.1 mostra os esforços e o ângulo $\theta_{\text {amin }}$ para os vários ângulos $\varphi$ adotados e a Figura 6.11 apresenta as armaduras resultantes para cada $\varphi$.

Tabela 6.1 - Esforços solicitantes equivalentes ao exemplo em 5.5 para vários ângulos $\varphi$

\begin{tabular}{|c|c|c|c|c|}
\hline$\varphi\left(^{\circ}\right)$ & $\mathrm{nxd}(\mathrm{kN} / \mathrm{m})$ & $\mathrm{nyd}(\mathrm{kN} / \mathrm{m})$ & $\mathrm{nxyd}(\mathrm{kN} / \mathrm{m})$ & $\theta$ amin $\left(^{\circ}\right)$ \\
\hline 0 & $-634,11$ & $-2065,89$ & 0,00 & 0,00 \\
\hline 4 & $-641,08$ & $-2058,92$ & 99,63 & 5,70 \\
\hline 8 & $-661,84$ & $-2038,16$ & 197,33 & 11,41 \\
\hline 12 & $-696,00$ & $-2004,00$ & 291,18 & 17,15 \\
\hline 16 & $-742,89$ & $-1957,11$ & 379,36 & 22,94 \\
\hline 20 & $-801,60$ & $-1898,40$ & 460,17 & 28,78 \\
\hline 24 & $-870,98$ & $-1829,02$ & 532,01 & 34,70 \\
\hline 28 & $-949,68$ & $-1750,32$ & 593,50 & 40,51 \\
\hline 30 & $-992,05$ & $-1707,95$ & 619,98 & 41,19 \\
\hline 32 & $-1036,17$ & $-1663,83$ & 643,44 & 41,80 \\
\hline 34 & $-1081,82$ & $-1618,18$ & 663,76 & 42,37 \\
\hline 36 & $-1128,78$ & $-1571,22$ & 680,85 & 42,90 \\
\hline 38,95 & $-1200,00$ & $-1500,00$ & 700,00 & 43,62 \\
\hline
\end{tabular}




\begin{tabular}{|l|c|c|c|c|}
40 & $-1225,69$ & $-1474,31$ & 705,02 & 43,87 \\
\hline 42 & $-1275,17$ & $-1424,83$ & 711,97 & 44,33 \\
\hline 45 & $-1350,00$ & $-1350,00$ & 715,89 & 45,00 \\
\hline 48 & $-1424,83$ & $-1275,17$ & 711,97 & 45,67 \\
\hline 50 & $-1474,31$ & $-1225,69$ & 705,02 & 46,13 \\
\hline 52 & $-1523,19$ & $-1176,81$ & 694,63 & 46,38 \\
\hline 54 & $-1571,22$ & $-1128,78$ & 680,85 & 47,10 \\
\hline 56 & $-1618,18$ & $-1081,82$ & 663,76 & 47,63 \\
\hline 58 & $-1663,83$ & $-1036,17$ & 643,44 & 48,20 \\
\hline 60 & $-1707,95$ & $-992,05$ & 619,98 & 48,81 \\
\hline 62 & $-1750,32$ & $-949,68$ & 593,50 & 49,49 \\
\hline 66 & $-1829,02$ & $-870,98$ & 532,01 & 55,30 \\
\hline 70 & $-1898,40$ & $-801,60$ & 460,17 & 61,22 \\
\hline 74 & $-1957,11$ & $-742,89$ & 379,36 & 67,06 \\
\hline 78 & $-2004,00$ & $-696,00$ & 291,18 & 72,85 \\
\hline 82 & $-2038,16$ & $-661,84$ & 197,33 & 78,59 \\
\hline 86 & $-2058,92$ & $-641,08$ & 99,63 & 84,30 \\
\hline 90 & $-2065,89$ & $-634,11$ & 0,00 & 90,00 \\
\hline
\end{tabular}

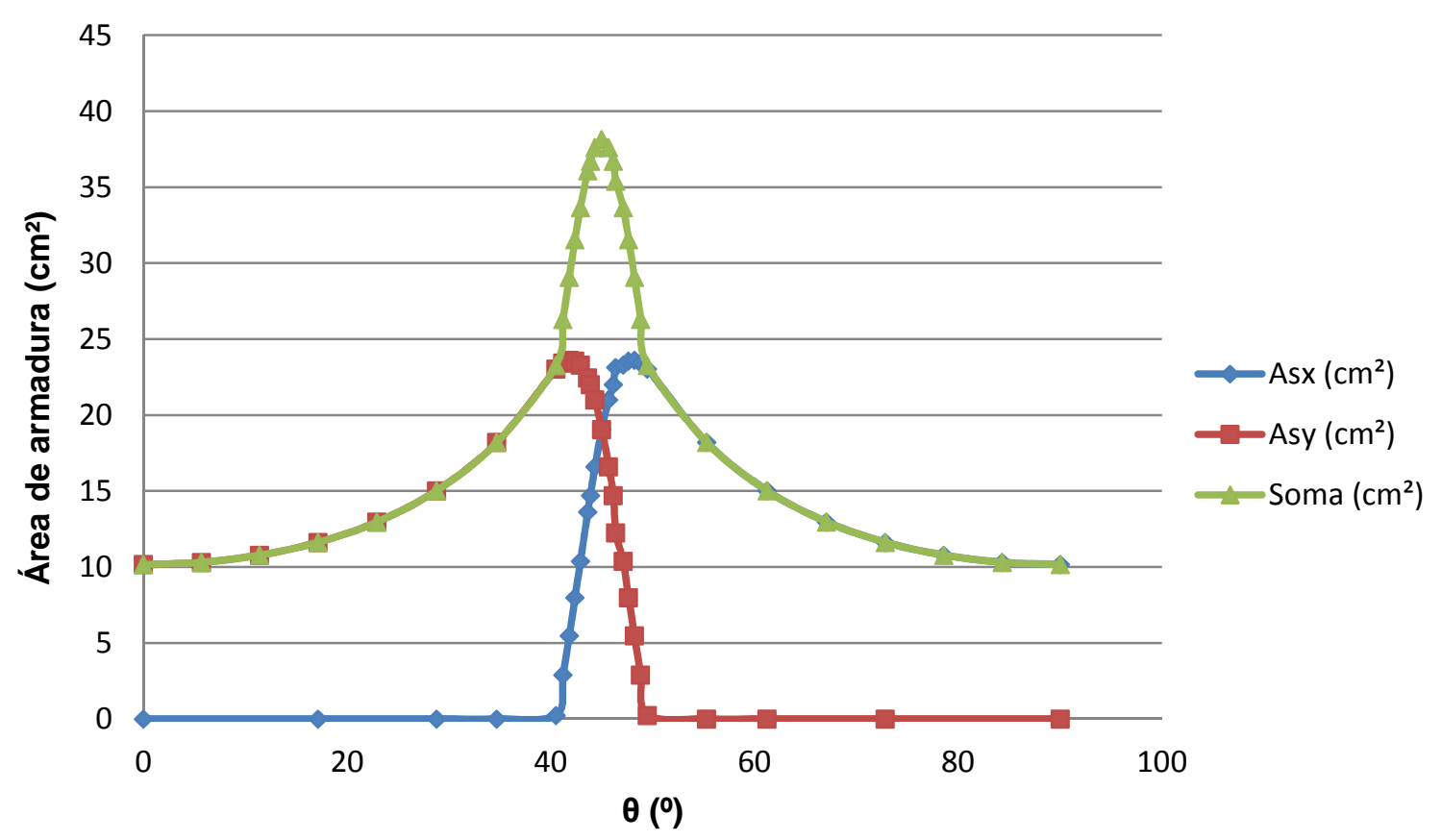

Figura 6.11 - Gráfico da área de armadura em função de $\theta$ correspondente a Tabela 6.1

A partir desses resultados, percebe-se que para $\varphi=0^{\circ}$ e $\varphi=90^{\circ}, \theta_{\text {amin }}=\varphi$. Isso é muito razoável já que se $\varphi=0$, as direções das armaduras coincidem com as 
direções principais. Pode se ver também pela Figura 6.11 que em $\theta_{\text {amin }}=\varphi=0^{\circ} \mathrm{e}$ $\theta_{\text {amin }}=\varphi=90^{\circ}$, as armaduras obtidas são as mínimas possíveis.

Já para qualquer $\varphi \neq 0^{\circ}$ ou $\varphi \neq 90^{\circ}, \theta \neq \varphi$. Isso acontece porque como as direções das armaduras para esses casos não coincide com as direções principais dos esforços solicitantes, e como ângulo $\theta_{\text {amin }}$, diferentemente de $\varphi$, é influenciado pelos esforços nas armaduras, $\theta \neq \varphi$. Somente para $\varphi=45^{\circ}$ que $\theta=45^{\circ}$ devido à simetria que aparece neste caso. 


\section{CONCLUSÕES}

Foram apresentados neste trabalho métodos para determinar as armaduras de compressão para o caso II, III e IV de dimensionamento, previstos pelo CEB (1990). Também foram apresentados os limites para este dimensionamento, ou seja, delimitaram-se os casos em que é possível adotar armaduras de compressão de forma com que a tensão de compressão no concreto seja reduzida para até a sua capacidade resistente. Percebe-se para todos os casos que estes limites estão relacionados apenas com o esforço de cisalhamento para o qual a chapa está submetida.

Além disso, foi utilizado um modelo resistente para o concreto que interpola os valores de resistência entre $f_{c d 1}$ e $f_{c d 2}$ de acordo com a curva obtida por Vecchio e Collins (1986), de forma que não haja descontinuidade de resistência entre os casos II e IV e casos III e IV. Também se mostrou como avaliar se a tensão de compressão no concreto está abaixo do limite para este modelo resistente. Para isto, foi necessário utilizar método iterativo para resolução das equações do problema, ficando como sugestão para futuros trabalhos a determinação da resolução analítica. Devido a este modelo adotado para o concreto, para os casos II e III, O dimensionamento das armaduras se tornou mais complexo e foram necessários métodos iterativos de resolução. Entretanto, ele leva a quantidades de armadura menores do que aquelas encontradas quando se usa somente os valores sugeridos pelo CEB (1990) para a resistência. Sugere-se para próximos trabalhos a determinação analítica dos valores de $\theta$ para estes casos.

Percebe-se que nos casos II e III os esforços normais só afetam as armaduras que estão em sua própria direção. Além disso, os esforços nas armaduras variam linearmente com os esforços normais, diferentemente do esforço de cisalhamento. Foram apresentados limites matemáticos para este esforço, porém para determinados níveis de cisalhamento, a área de armadura pode assumir valores próximos a infinito, o que é fisicamente impossível. Portanto, devem-se levar em conta os limites de armaduras máximas determinados pela NBR 6118 .

Quanto ao caso IV, verificou-se que existem infinitas soluções para o dimensionamento das armaduras, embora somente uma leve à armadura mínima 
necessária. Isto acontece devido ao menor número de variáveis fixas, se comparado aos casos II e III. Só foi possível encontrar a solução para este dimensionamento econômico por meio de tentativas. Não foi possível determinar analiticamente qual é a solução que resulta na armadura mínima para este caso, ficando como sugestão para futuros trabalhos.

Também para o caso IV, nota-se que a variação do esforço normal em uma direção afeta a área de armadura necessária na outra direção, diferentemente dos casos II e III. Isto se deve a possibilidade da variação das deformações em x e y, de forma a se obter a armadura mínima.

Sugere-se para futuros trabalhos, além do já exposto, a comparação dos resultados obtidos pelos métodos de dimensionamento apresentados neste estudo com modelos físicos e/ou numéricos.

Também se sugere para próximos estudos a elaboração de uma rotina de cálculo automatizada que implemente os conceitos aqui expostos. 


\section{REFERÊNCIAS BIBLIOGRÁFICAS}

ABNT. Norma Brasileira NBR-6118: Projeto de estruturas de concreto. Rio de Janeiro, 2003.

ACI COMITTEE 318. Building code requirements for structural concrete. American Concrete Institute, Detroit, 2011.

BENTZ, EVAN C.; VECCHIO, F. J.; COLLINS M. P. Simplified modified compression field for calculating shear strength of reinforced concrete Elements, ACI Structural Journal. July-August, 2006, p.614-624.

BHIDE, S. B..; COLLINS M. P. Influence on the shear capacity of reinforced concrete members, ACI Structural Journal. September-October, 1989, p.570-581.

CHEN, REINALDO. Dimensionamento de elementos de superfície de concreto Armado: membranas, placas e cascas. 2004. 148 p. Dissertação (Mestrado) Escola Politécnica da Universidade de São Paulo, São Paulo, 2004.

COMITÉ EURO-INTERNATIONAL DU BETÓN. CEB-FIP model code 1990. London, Thomas Telford, 1993

Bulletin d'Information, n.223: A state-of-art report. Ultimate limit state design of structural concrete shell elements. June, 1995.

COMITÉ EUROPÉEN DE NORMALISATION. Eurocode2: Design of concrete structures. Brussels, 1999.

CORRÊA, M. R. S; RAMALHO M.A. Análise de Estruturas de Concreto. Simpósio EPUSP sobre estruturas de concreto, 3, São Paulo, 1993. Anais. São Paulo, 1993. p. 151-172.

DELLA BELLA, J. C.; CIFÚ, S. Critérios para dimensionamento das armaduras e verificação do concreto em estruturas laminares submetidas a solicitações de chapa e placa. In: Simpósio EPUSP sobre estruturas de concreto, 4, São Paulo, 2000. Anais. São Paulo, 2000. 
FIALKOW, M.N. Strength design of shell membrane reinforcement. Journal of Structural Engineering, v. 109, n. 4, 1983. p. 891-908.

FUSCO, P. B. Técnicas de armar as estruturas de concreto. São Paulo, Editora Pini, 1994.

GUPTA, AJAYA K. Combined membrane and flexural reinforcement in plates and shells. Journal of Structural Engineering, v. 112, n. 3, March, 1986. p. 550-557.

Membrane reinforcement in shells. Journal of the Structural Division, Proceedings of the American Society of Civil Engineers, v.107, January, 1981. p. 4156.

. Membrane reinforcement in shells: a review. Nuclear Engineering and Design, v.82, January,1984. p. 63-75.

GUPTA, AJAYA K; AKBAR H. Cracking in reinforced concrete analysis. Journal of Structural Engineering, v. 110, n. 8, 1984. p. 1735-1746.

JAZRA, F. M. Dimensionamento de chapas de concreto armado. 2008. 126 p. Dissertação (Mestrado) - Escola Politécnica da Universidade de São Paulo, São Paulo, 2008.

LEONHARDT, F.; MONNIG, E. Construções de concreto v. 2: casos especiais de dimensionamento de estruturas de concreto armado, Rio de Janeiro, Interciências, 1978.

LOURENÇO, P. B.; FIGUEIRAS, J. A. Solution for the design of reinforced Concrete plates and shells. Journal of Structural Engineering, v. 113,n. 5, May, 1995. p. 815-823.

VECCHIO, F. J.;COLLINS M. P. The Modified compression-Field theory for reinforced concrete elements subjected to shear. ACI Structural Journal. MarchApril, 1986, p.219-231 